\title{
CONCERNING THE DECOMPOSITION AND AMALGAMA- TION OF POINTS, UPPER SEMI-CONTINUOUS COLLECTIONS, AND TOPOLOGICAL EXTENSIONS
}

BY

\author{
R. G. LUBBEN
}

1. Introduction. A necessary and sufficient condition that a space $H$ Fréchet have the Borel-Lebesgue covering property is that each monotonic collection of closed point sets in it have a nonvacuous product $\left({ }^{1}\right)$. Thus, if such a space is not perfectly compact, there exists in it a monotonic collection of closed sets, $E$, with a vacuous product; $E$ is an example of what we call a boundary element. With the help of $E$ we may define a boundary point, $P(E)$; if $P(E)$ is added to the basic space and a suitable topology is introduced, the closures of the set of elements of $E$ contain $P(E)$; and the deficiency of the elements of $E$, that their product is vacuous, no longer holds for the aggregate of their closures in the extended space. By adding to $S$ an aggregate of boundary points which satisfy suitable conditions we achieve the embedding of $S$ in a perfectly compact Hausdorff space (cf. Theorem 16.1).

In Chapter I we introduce the class of point elements; these include the boundary elements. We define the relation of the intersection of two point elements; in terms of this we give an ordering which makes the aggregate of all point elements a quasi-partially ordered system. A repetition of this algorithm gives the quasi-partially ordered system $M$ of all collections of point elements. By a process identifying the equivalent elements of $M$ we obtain a partially ordered system whose elements we call portions of our basic space $S$, or $S$-portions; these are our ideal points. A relation of an open set $D$ in $S$ and an ideal point $P$ is expressed by saying that $D$ is an $S$-neighborhood of $P$; the $S$-neighborhoods are used in topologizing collections of ideal points. The relation $P$ is an end of $M$ between the ideal point $P$ and the closed point set of $S, M$,

Presented to the Society, in three parts: September 9, 1937, under the title Perfectly compact Hausdorff spaces in which a normal space may be embedded; December 28, 1937, under the title Concerning upper semi-continuous collections and the decomposition of points of normal spaces; and September 6,1938, under the title The extension of homeomorphisms of normal spaces to topologically related spaces; received by the editors July 18, 1938, and, in revised form, September 9, 1940.

(1) Cf. Moore, (II) (the bibliography is at the end of the paper). Kuratowski and Sierpinski, loc. cit., show that the Borel-Lebesgue property is equivalent to the condition that each infinite point set have a complete limit point. Fréchet, (II), calls this property perfect compactness; several years later Alexandroff and Urysohn, (I), call it bicompactness.

A collection of sets is said to be monotonic provided that if $K$ and $L$ are two of its elements, then either $K \supset L$ or $L \supset K$; Moore, loc. cit. 
suggests an analogy between Carathéodory's theory of ends and ours in that both are concerned with methods of approach or accessibility. Our end-theory is used as a technique in developing that of the $S$-neighborhoods.

We shall now indicate some of the ideas underlying the methods which we discussed in the preceding paragraph. The theory of point elements and that of ends suggest our regarding a point not as a static entity but rather as that of a relation of the point to the remainder of space, that involves methods of approach. Secondly, a point need not necessarily be indivisible. We have a conception according to which our space $S$ consists of a quantity of basic matter; this may be decomposed and put together in various ways, and the same applies for each portion of this matter. In particular, the points of $S$ are subject to such operations. There exists a maximal portion, that of all the matter; there prove to be atomic portions.

These ideas may be described by means of our ordered system of ideal points; let " $A<B$ " be the ordering relation of this system. Let $P$ be an ideal point and $M$ be a collection of ideal points; if both $P<M$ and $M<P$, we shall think of $P$ as a summation or an amalgamation of $M$, and of $M$ as a breaking up or a decomposition of $P$; we think of $M$ and $P$ as describing different structures of the same portion of the basic matter of $S$. Let $X$ and $Y$ be collections of $S$-portions and " $y=\alpha(x)$ " be a transformation from $X$ to $Y$ such that for $y_{1} \in Y, y_{1}$ is the amalgamation of the elements of $\alpha^{-1}\left(y_{1}\right)\left({ }^{2}\right)$; in proceeding from $\alpha^{-1}\left(y_{1}\right)$ to $y_{1}$ we are involved in a change of structure, only; and, thus, this transformation may be said to preserve $S$-portions or to leave $S$-portions invariant.

If we were concerned with order relations, only, the system of all our ideal points would afford a satisfactory basis for the amalgamation and decomposition theory. From a topological standpoint, however, we require systems of elements for which the $S$-portion preserving transformations, $y=\alpha(x)$, which describe the amalgamation and the decomposition processes, satisfy continuity conditions. As illustrations of systems which meet this requirement we refer to a result by Stone $\left({ }^{3}\right)$. Of particular interest is the case where $X$ and $Y$ are perfectly compact Hausdorff spaces; this case leads us to apply a mapping theory by Kolmogoroff, which, for our purposes, finds a summary in Theorem VIII, p. 98, Alexandroff and Hopf, loc. cit. The adaption of this theory to the $S$-portion preserving transformations leads to the introduction of a special class of ideal points, the amalgamation points. It is interesting to note that since in applications we are to use both order and continuity relations, such relations are used in defining these points. Chapter II is devoted mainly to a development of the properties of amalgamation points; such properties have striking analogies to those of closed point sets in perfectly compact Hausdorff spaces. For instance, a decomposition of an amalgamation point into atomic

(2) The symbol, $\alpha^{-1}\left(y_{1}\right)$ means all elements of $X, x_{1}$, such that $y_{1}=\alpha\left(x_{1}\right)$.

( ${ }^{3}$ Loc. cit., p. 476, Theorem 88 ; we discuss this in $\$ \S 16$ and 20. 
$S$-portions is a perfectly compact set (cf. Theorem 14.4). In $\$ 15$ this analogy is shown to be fundamental; here we show that the decomposition $M$ of an amalgamation point $P$ into regular amalgamation points is perfectly compact if and only if it is upper semi-continuous relative to $S$; we develop an elaboration of the Kolmogoroff-Alexandroff-Hopf theory of the S-portion preserving continuous transformations of such decompositions.

The sections following $\$ 15$ are devoted to applying these results. In Chapter III we investigate systems of such applications. In $\$ 19$ we consider the ordered system $\delta(P)$ of all perfectly compact decompositions of $P$ into regular amalgamation points; the ordering " $X<Y$," which means that $X$ may be mapped on $Y$ by a continuous, $S$-portion preserving mapping $y=\alpha(x)$, makes $\delta(P)$ a complete lattice. If $\delta$ is a sublattice of $\delta(P)$, the sum of the elements of $\delta, L_{\delta}\left({ }^{4}\right)$, becomes a lattice of amalgamation points. The zero of $\delta, \omega_{\delta}$, is the system of atomic elements of $L_{\delta}$, and may be mapped on each element of $\delta$. Such $\omega_{\delta}$ 's may be described as universal inverse mapping spaces relative to the elements of $\delta$. Particularly important is the case for which $P$ is the maximal amalgamation point; that is, $P$ is the amalgamation of all our ideal points. Then we let $\delta(S)=\delta(P)$; in $\$ 20$ we show that the completely regular spaces $S$ are characterized by the property that there exist elements of $\delta(S)$ in which the decomposition of $S$ has proceeded to elements at least as small as the points of $S$. For such a space we have the interesting subsystem of $\delta(S)$, $\delta=H(S)$, which consists of the images of all immediate extensions of $S$ which are perfectly compact Hausdorff spaces. The zero of $H(S), \lambda(S)$, is the topological image of the space considered by Stone in his Theorem 88 (loc. cit., p. 476). In $\$ 17$ the space $\lambda(S)$ enters into an interesting characterization of the normality of $S$; this is given by the condition that each upper semi-continuous decomposition of $S$ into a collection of closed point sets can be extended to a similar decomposition of $\lambda(S)$. Further, only the topological images of $\lambda(S)$ have this property.

In Chapter II we introduce the concept of a semi-completely normal space. For such a space the theory of Chapter III is especially simple; such a space is characterized by the property that the zero of $\delta(P), \omega(P)$, is a set of atomic ideal points. Similarly, the normal space is characterized by the condition that the only boundary points which are elements of an $\omega(P)$ are atomic points. In Chapter II we give characterizations of various types of normal and regular spaces.

\section{Chapter I. AN ORdered System of IDEAL POINTS}

At the beginning of the preceding section we discussed the methods and the underlying ideas which occur in the development of our theory of point

(4) It may be necessary to add a zero to $L_{\delta}$ to make it a lattice; such is not the case for $\delta(P)$, or for $\delta$. 
elements and ideal points. It may be mentioned that methods similar to those used in this chapter may be used to extend a partially ordered system $K$ to a complete partially ordered system, granting that $K$ satisfies suitable conditions.

2. Concerning a topological background. We refer the reader to treatises by Fréchet, Hausdorff, Menger, Moore, Sierpiński, and Alexandroff and Hopf (cf. Bibliography). Throughout the paper the symbol $S$ will denote a basic Hausdorff space. We shall consider only spaces $H$, Fréchet (cf. Fréchet, (I), p. 186). Alexandroff and Hopf call such spaces $T_{1}$ spaces (loc. cit., p. 59).

Definitions. D 2.1. The symbol $M_{T}^{\prime}$, the derived set of $M$ relative to $T$, means the set of all points of $T$ which are limit points of $M \cdot T$. The symbol $\bar{M}_{T}$ denotes the closure of $M$ relative to $T$; that is, $M \cdot T+(M \cdot T)_{T}{ }^{\prime}$.

D 2.2. If $T$ and $R$ are spaces (1) such that $T \supset R$ and (2) such that if $M$ is a point set in $R$ then $\bar{M}_{R}=R \cdot \bar{M}_{T}$, then we say that $R$ is a subspace of $T$, that $R$ is embedded in $T$, and that $T$ is an extension of $R$; if, in addition, $T$ is a Hausdorff space, it is a Hausdorff extension of $R$. If $T=\bar{R}_{T}, T$ is an immediate extension of $R$ (cf. Stone, loc. cit., p. 420).

D 2.3. If $T$ is an immediate Hausdorff extension of $S$, the points of $T-S$ are called frontier points of $S$. If $P$ is a point of $T, T \supset S, D$ is an open set of $S$, and $P$ does not belong to the closure in $T$ of $S-D$, we call $D$ an $S$-neighborhood of $P$. Two frontier points of $S$ are said to intersect provided that each $S$-neighborhood of the one contains points in common with each $S$-neighborhood of the other.

3. Partially ordered and quasi-partially ordered systems. We refer the reader to a treatise by Birkhoff and an article by MacNeille for discussions, examples, and references to the literature (cf. Bibliography).

D 3.1. By a partially ordered system $\left(^{(5)}\right.$ is meant a system $X$ in which an (ordering) relation " $x \leqq y$ " is defined which satisfies the following conditions: $\left(\mathrm{P}_{1}\right)$ For $x \in X, x<x ;\left(\mathrm{P}_{2}\right)$ if $x<y$ and $y<x$, then $x=y$; $\left(\mathrm{P}_{3}\right)$ if $x<y$ and $y<z$, then $x<z$. If the condition $\mathrm{P}_{2}$ is omitted, then $X$ is a quasi-partially ordered system. The notation " $x<y$ " will be read " $y$ includes $x$," " $x$ precedes $y$," or " $x$ is a part of $y$." If $x<y$ but not $y<x$, we say that $x$ is a proper part of $y$.

D 3.2. If $x<y$ and $y<x$, we say that $x$ and $y$ are equivalent (relative to the ordering " $<$ "). Note that $\mathrm{P}_{2}$ rules out distinct equivalent elements. In this paper we shall have many examples of quasi-partially ordered systems for which $\mathrm{P}_{2}$ does not hold.

D 3.3. An element of $X$ is said to be atomic, if no element of $X$ is a proper part of it.

D 3.4. An element of $X$ is a lower bound of a subcollection of $X, N$, if it is a part of each element of $N$. The set of all lower bounds of $N$ is called the

(5) Cf. Hausdorff, (I), p. 139 and Birkhoff, pp. 5, 7. 
intersection $I(N)$ of the elements of $N$. A greatest element of $I(N)$ is called a greatest lower bound of the elements of $N$. The terms upper bound and least upper bound have analogous definitions. A zero and a unit of $X$ are, respectively, a lower and an upper bound of all the elements of $X$.

D 3.5. If two elements of $X$ have a lower bound in $X$, they are said to intersect (in $X$ ).

D 3.6. A subcollection of $X, N$, is said to be monotonic if for $x \in N$ and $y \in N$ either $x<y$ or $y<x$.

D 3.7. If $X$ is partially ordered and each subcollection of $X$ has a greatest lower bound in $X, X$ is a complete multiplicative system.

D 3.8. A lattice is a partially ordered system $X$ such that any two of its elements have both a greatest lower bound and a least upper bound in $X$; if each subset of $X$ has such bounds, $X$ is complete.

4. The point elements. In $\$ 1$ we discussed the origin of our concept of a boundary element. This discussion applies also to a decomposition point element which satisfies (A) of D 4.1. If $M=a(E)$ is a non-isolated point of $S$, (4) of D 4.1 guarantees that $E$ assist in the decomposition of $M$ according to the theory we are to develop. For Case (A) the elements of $E$ are subsets of $S-a(E)$; this is desirable for technical reasons in expressing relations of a point element to its $S$-neighborhoods (cf. D 4.5 and D 11.1). The fact that the topological relations of $M=a(E)$ to point sets in $S$ depend on subsets of $S-M$ gives reasons that $S-M$ should contain $S$-neighborhoods of $E$.

D 4.1. A boundary element of $S$ is a nonvacuous collection $E$ such that (1) each of its elements is a nonvacuous, closed point set of $S,(2)$ the product of any two elements of $E$ contains an element of $E$, and (3) the product of all elements of $E$ is vacuous. A collection of sets $E$ is called a point element of $S$ if either (A) or (B) is satisfied: (A) Either $E$ is a boundary element of $S$, or there exists a non-isolated point of $S, M$, such that $E$ is a boundary element of the subspace of $S, S-M$, and the following condition holds: (4) each open set in $S$ that contains $M$ contains an element of $E\left({ }^{6}\right)$. (B) There exists an isolated point of $S, M$, which is an element of $E$; each element of $E$ is a closed point set of $S$ which contains $M$. If $E$ satisfies (B) it is said to be degenerate.

For Case (B) $E$ satisfies conditions (1), (2), and (4), and the product of all elements of $E$ is $M$. For Case (A) the product of all elements of $E$ is vacuous, and (4) implies (3).

D 4.2. Let $a(E)=M$ be the product of the closures in $S$ of the elements of $E$. We say that $a(E)$ is attached to $E$, and conversely.

$\left(^{6}\right)$ The two cases under (A) may be combined as follows; this is the form of (A) we shall use in our applications: There exists an $M$, which is either a non-isolated point of $S$ or is the null set, such that (1) each element of $E, e$, is a nonvacuous, closed point set in the space $S-M$, and its closure in $S$ is $M+e$; (2) the product of two elements of $E$ contains an element of $E$; (3) the product of all elements of $E$ is a subset of $M$; (4) if $M$ is a point of $S$, any open set of $S$ which contains $M$ contains an element of $e$. 
D 4.3. If $a(E)$ is a point of $S, E$ is called a decomposition point element. If $E$ is a boundary element, $a(E)$ is vacuous.

D 4.4. Two point elements are said to intersect, provided that each element of the one has points in common with each element of the other.

D 4.5. An open set in $S$ which contains an element of a point element $E$ is called an $S$-neighborhood of $E$. A point or a frontier point of $S$ is said to intersect $E$, provided that each $S$-neighborhood of the point has points in common with each $S$-neighborhood of $E$. (Cf. D 2.3.)

D 4.6. If $E$ and $F$ are point elements and each point element which intersects $E$ intersects $F$, we say that " $E<F$ "; we read this " $E$ is a portion of $F$ (relative to $S$ )."

$D$ 4.7. Let the aggregate of all point elements be ordered by the relation " $E$ is a portion of $F$." It is easy to see that $\mathrm{P}_{1}$ and $\mathrm{P}_{3}$ of $\mathrm{D} 3.1$ are satisfied but that $\mathrm{P}_{2}$ is not. Thus, this system is quasi-partially ordered.

Examples. E 4.1. Let $S$ be the Euclidean plane, $M$ be the origin, and $P_{n}$ be the point with coordinates $(1 / n, 0)$. Let $e_{n k}=\left(P_{n}, P_{n+k}, P_{n+2 k}, \cdots\right)$, and $E_{j k}=\left(e_{j k}, e_{j+k, k}, e_{j+2 k, k}, \cdots\right)$. Then the $E$ 's are decomposition point elements of $S$ and are attached to $M$; also, they are boundary elements of $S-M$. $E_{12}$ and $E_{22}$ do not intersect, but each is a portion of $E_{11}$. $E_{33}$ intersects each of $E_{12}$ and $E_{22}$; none of these point elements is a portion of any of the others. $E_{66}$ is a greatest lower bound of $E_{22}$ and $E_{33}$; and $E_{36}$ is a greatest lower bound of $E_{12}$ and $E_{33}$.

E 4.2. Let $S$ be a space with infinitely many points, all of which are isolated. Then all decomposition point elements are degenerate. Any monotonic collection of point sets whose elements have a vacuous product is a boundary element.

E 4.3. Let $H$ be a continuum in the plane whose points have coordinates $(x, y)$ which satisfy one of the following conditions: (1) $0 \leqq x \leqq 1, y=0$; (2) $x=1 / k$ and $0<y \leqq 1 / k$, where $k=1,2,3, \cdots$. Let $F$ be the aggregate of all subcontinua of $H$ which contain the origin and at least one point with a positive ordinate. Let $M$ be the origin and $E$ be the aggregate of all sets $f-M$, where $f \in F$. Let $G$ be the aggregate of all sets obtained by reflecting elements of $E$ in the $X$ axis. Then $E$ and $G$ are equivalent point elements. However, no element of either $G$ or $E$ is a subset of an element of the other.

5. The intersection of point elements; atomic elements. Because of Theorem 5.1 the definitions which are given for the intersection of point elements in D 3.5 and D 4.4 are logically equivalent. An analogous state of affairs shows that such a consistency should not be considered obvious: Let $K$ be a partially ordered system; define for $K$ a new order by an application of Definitions D 3.5, D 4.6, and D 4.7; the new order need not be consistent with the basic order of $K$.

THEOREM 5.1. In order that two point elements $E$ and $F$ should intersect, it 
is necessary and sufficient that there exist a point element which is a portion of both; if this condition is satisfied and $G$ is the aggregate of all sets which are the product of an element of $E$ with an element of $F$, then (a) $G$ is a point element and a greatest lower bound of $E$ and $F$, and (b) $a(E)=a(F)=a(G)$.

Proof. Let $H$ be a common portion of $E$ and $F$. Since $H$ intersects itself, it intersects each of $E$ and $F$. Since $E$ intersects $H$ and $H$ is a portion of $F$, $E$ intersects $F$.

Conversely, let $E$ and $F$ intersect. Suppose that $a(E)$ is a point of $S$ and $a(E) \neq a(F)$. By D 4.1, (3), there exists $f \in F$ such that $f$ does not contain $a(E)$. Since $\bar{f}=f+a(F), a(E) \in S-\bar{f}$; by D 4.1, (4), S- $\bar{f} \supset e \in E$; since $e \cdot f$ is vacuous, we are involved in a contradiction. Thus, either $a(E)$ and $a(F)$ are the same point of $S$, or both are the null set. If $E$ and $F$ are degenerate, the conclusion in (a) is obvious; cf. D 4.1, (B). Suppose, therefore, that neither is degenerate. Let $g_{1}=e_{1} \cdot f_{1}$ and $g_{2}=e_{2} \cdot f_{2}$ be elements of $G$, and $K=a(E)$. Then $g_{1} \cdot g_{2}=\left(e_{1} \cdot f_{1}\right) \cdot\left(e_{2} \cdot f_{2}\right)=\left(e_{1} \cdot e_{2}\right) \cdot\left(f_{1} \cdot f_{2}\right) \supset e_{3} \cdot f_{3}=g_{3} \in G ;$ thus, Condition (2) of D 4.1 is satisfied; clearly the same holds for (3) and (4). Because of (2) and (4) $\bar{g}_{1} \supset g_{1}+K$; the converse is true, since $\bar{e}_{1}=e_{1}+K$ and $\bar{f}_{1}=f+K$. Thus, $G$ satisfies all the conditions of D 4.1, (A), and it is a point element. Let $H$ be a point element which intersects $G, h \in H, e \in E$, and $f \in F$. Then $h \cdot(e \cdot f)$ is nonvacuous. It follows that each of $e \cdot h$ and $f \cdot h$ is nonvacuous. Thus, $H$ intersects each of $E$ and $F$, and $G$ is a common portion of $E$ and $F$.

Let $X$ be a point element which is a common portion of $E$ and $F$, and $Y$ be a point element which intersects $X$. Let $e, f, x$, and $y$ be elements, respectively, of $E, F, X$, and $Y$. Let $Z$ be the aggregate of all products of an element of $X$ by an element of $Y$; similarly define $T$ in terms of $E$ and $Z$. By the preceding paragraph $Z$ is a point element and $Z<X$; since $X<E, Z$ intersects $E$, and $T$ is a point element. Then $T<Z<X$ and $T$ intersects $X$. Since $x \cdot y \cdot e \in T$ and $X<F, f \cdot(x \cdot y \cdot e)$ is nonvacuous. Then $y \cdot(e \cdot f)$ is nonvacuous; since $e \cdot f \in G$, $Y$ intersects $G$. Thus, $X<G$, and $G$ is a greatest lower bound of $E$ and $F$.

TheOREM 5.2. No decomposition point element intersects a boundary element.

TheORem 5.3. A degenerate point element is atomic.

THEOREM 5.4. If $M$ is a collection of point elements and each finite subcollection of $M$ has a lower bound, then $M$ has a greatest lower bound.

This lower bound may not belong to $M$. The theorem has an analogy to conditions for perfect compactness; cf. Moore, (II), and Fréchet, (I), p. 231.

Proof. Let $H$ be the aggregate of all point elements $E$ such that $E$ is a greatest lower bound of a finite subcollection of $M$; if $m \in M, m<m$; thus, $m \in H$ and $H \supset M$. Let $K$ be the sum of the elements of $H$, and $e$ and $f$ be elements of $K$. There exist $E \in H$ and $F \in H$ such that $e \in E$ and $f \in F$. There exist two finite subcollections of $M, H_{E}$ and $H_{F}$, such that $E$ and $F$ are greatest 
lower bounds, respectively, of $H_{E}$ and of $H_{F}$. By our condition $T=H_{E}+H_{F}$ has a lower bound; let $\beta$ be any lower bound of $T$. Then $\beta$ is a lower bound of $H_{E}$, of $E$, of $H_{F}$, of $F$, and of $G$, where $G$ is a greatest lower bound of $E$ and $F$ (by Theorem $5.1 E$ and $F$ have a greatest lower bound). Since $\beta$ is $a n y$ lower bound of $T, G<E$, and $G<F$, then $G$ is a greatest lower bound of $T$; then $G \in H$ and $K \supset G$. By Theorem $5.1 e \cdot f \in K$. Thus, Condition (2) of D 4.1 is satisfied by $K$. By Theorem $5.1 a(E)=a(F)=a(G)$. It follows readily that the other conditions of D 4.1 are satisfied. Since each element of $M$ is a subset of $K, K$ is a portion of each of these elements. Thus, $K$ is a lower bound of $M$. If the point element $F$ is a lower bound of $M$, it follows from the definition of $H$ that $F$ is a portion of each element of $H$. If a point element intersects $F$, it intersects each element of $H$, and thus intersects $K$. Thus, $F<K$, and $K$ is a greatest lower bound of $M$.

Theоrem 5.5. If $E$ is a point element, there exists an atomic point element which is a portion of $E$.

Proof. There exists a monotonic collection of point elements, $M$, of which $E$ is an element, which is not a proper subcollection of any monotonic collection of point elements; cf. Hausdorff, (I), p. 140. Let $F$ be a greatest lower bound of $M$, and $G$ be a point element such that $G<F$ (cf. Theorem 5.4). Then $G+M$ is monotonic, $G \in M$, and $F<G$; since $G<F$, by $\mathrm{D} 3.3 F$ is atomic.

5A. Historical. In $\$ \$ 16,17$, and 20 we discuss the space $\lambda(S)=S+M$, where $S$ is a completely regular space, $M$ is a collection of regular boundary points which are the atomic elements of a certain system, and $\lambda(S)$ is perfectly compact. This space has been studied by Stone, Wallman, and Cech $\left(^{7}\right)$. Cech, on page 833, has a result which is equivalent to the following: In order that $S$ be normal, it is necessary and sufficient that if $F_{1}$ and $F_{2}$ are mutually exclusive closed point sets in $S$, and $\beta \in \lambda(S)-S$, then $\beta$ is not a limit point of both. An analogous condition on one of our ideal points $\beta$ is necessary and sufficient that $\beta$ be atomic $\left.{ }^{8}\right)$; this is true even if $S$ is irregular. By Theorem 16.1 it is characteristic of a normal space that the elements of $\lambda(S)-S$ be atomic boundary points. Thus, Cech encounters the atomic points only in the case of the normal space, while we have them at our disposal for any Hausdorff space. This difference seems to be characteristic of the difference of our methods; here Čech seems to follow Tychonoff and Urysohn. Stone's methods involve an extensive use of algebra, but seem to have elements in common with those of Cech.

Wallman's methods most nearly resemble ours. He constructs the points

( ${ }^{7}$ Cf. bibliography. Stone's paper appeared in May, 1937. The author's first report before the Society was given in September, 1937; it included most of the results of the first two chapters of the present paper for the case of the normal and semi-completely normal spaces. The papers of Cartan, Wallman, and Čech appeared later.

(8) Compare the comment on Theorem 11.7 and Theorem 12.3. 
of $\lambda(S)$ by means of collections of sets; for the case of the boundary points he uses what we should call atomic boundary elements. For the other type of points, those corresponding to the points of $S$, he uses definitions which are not suitable for our decomposition theory. Since he does not consider such a theory, his definitions are adequate for his purposes.

Cartan, on the other hand, has a basis for a decomposition theory, based on order, resembling that developed in our $\$ 5$; however, he fails to develop the topological applications, and has nothing like the order-continuity theory of our Chapters 2 and 3. His filter bases and filtres correspond to our point elements and composition points; he proves the existence of ultra-filtres which correspond to our atomic points. He has the theorem: In order that two filter bases should generate the same filter, it is necessary and sufficient that each element of the one should contain an element of the other. By Example E 4.3 such a theorem is not true for our point elements. Thus, formally his definitions are not quite adequate for our treatment.

For a domain in the plane Carathéodory has a theory of chains, ends, and prime ends, which correspond to our boundary elements, boundary points, and atomic boundary points. His treatment of accessibility corresponds to our sections 6 and 10.

6. A Theory of accessibility. The overlapping of a point element and a closed set in $S$, which we define in D 6.1, resembles the intersection of two point elements (cf. D 4.4). Similarly, Theorem 6.1 is an analogue of Theorem 5.1. The discussion at the end of $\$ 5 \mathrm{~A}$ suggests an interpretation of the results of Theorem 6.1 according to which a point element involves methods of approach or of accessibility. A boundary element may be regarded as a "way" for escaping from the basic space; the corresponding boundary point serves as a barrier for such an exit, and may be approached by such a way. In D 6.2 we formulate these ideas.

D 6.1. If $\mathrm{E}$ is a point element, $M$ is a point set in $S$, and each element of $E$ has points in common with $M$, then $E$ and $M$ are said to overlap.

D 6.2. Let $E$ and $F$ be point elements and $M$ be a point set in $S$. If each point element which intersects $E$ also intersects $F$ or overlaps $M$, respectively, we say that $E$ is a way in $F$, or that $E$ is a way in $M$, respectively. Clearly, the former is equivalent to the relation " $E<F$ "; the latter is analogous.

Examples. E 6.1. Let $S$ be the plane, $M$ be a line, $Q$ be a point of $M$, and $E$ be the aggregate of all sets $I-Q$, where $I$ is an interval on $M$ and $Q$ is its midpoint. Then $E$ is a maximal common way of $M$ and $E$, and also of $S$ and $E$. If $R_{1}$ and $R_{2}$ are the two rays on $M$ from $Q$, then any way common to $R_{1}$ and $E$ fails to intersect any way common to $R_{2}$ and $E$. We might say that there are many more ways of approach to $Q$ in $S$ than there are in $M$; and there are more in $M$ than in $R_{1}$.

$E$ 6.2. In the notation of Example $E 4.1$ the point element $E_{36}$ is a maximal common way of the set $e_{33}$ and the point element $E_{12}$. 
Theorem 6.1. Let $M$ be a closed point set in $S$ and $E$ be a point element. (1) In order that there exist a way common to $M$ and to $E$, it is necessary and sufficient that $M$ and $E$ overlap; (2) if this condition is satisfied and $E(M)$ is the aggregate $[e \cdot M]$, where $e \in E$, then $E(M)$ is a maximal common way of $E$ and $M$ and $M \supset a(E(M))$.

The content of the theorem and its proof are similar to those of Theorem 5.1. In particular, replace the " $F$ " and the " $f$ " of that proof by " $M$," and replace " $G$ " by " $E(M)$."

TheORem 6.2. If $F$ is a portion of $E$ and $G$ is a way common to $M$ and $F$, then $G$ is a way common to $M$ and $E$.

7. The quasi-partial ordering of collections of point elements. As in $\$ 4$ we first define intersection and then define order in terms of intersection. The definitions of the two sections 4 and 7 are similar and the system of $\S 4$ is a subsystem of the one we consider here. In $\$ 1$ we have explained how the results of $\S \S 7$ and 8 afford a basis for a summation or an amalgamation theory, and how those of $\$ 4$ yield a decomposition theory.

D 7.1. If a point element intersects one of a collection of point elements, the point element and the collection are said to intersect. Two collections of point elements are said to intersect if one of them intersects an element of the other.

D 7.2. Let $A$ be the aggregate whose elements are (1) the point elements and (2) the collections of point elements. If $M$ and $N$ are elements of $A$ and each element of $A$ that intersects $M$ also intersects $N$, we say that $M$ is a portion of $N$ (relative to $S$ ), or $M<N$. Clearly, this relation is reflexive and transitive.

D 7.3. Let the relation " $M<N$ " order the elements of $A$. Clearly D 7.3 and D 4.6 are equivalent if both $M$ and $N$ are point elements. Thus the ordered system of the point elements of D 4.7 is a subsystem of $A$.

Example. E 7.1. Adopt the notation of E 4.1. Let $M=E_{11}, N=\left(E_{12}, E_{22}\right)$, $K=\left(E_{14}, E_{24}, E_{34}, E_{44}\right)$, and $L=\left(E_{14}, E_{34}\right)$. Then $M, N$, and $K$ are equivalent, and so are $L$ and $E_{12}$. Also, $L$ is a portion of $M$, but the converse is not true. $L$ does not intersect $E_{22}$. $L$ intersects $\left(E_{14}, E_{24}\right)$ but neither of these is a portion of the other.

D 7.4. If $M$ is a point element or a collection of point elements, let $\Sigma(M)$ denote the set of all point elements that are portions of $M$; let $\alpha(M)$ denote the set of atomic elements of $\Sigma(M)$.

THEOREM 7.1. If $M$ and $N$ are collections of point elements, then $M$ is a portion of $N$ if and only if each point element that intersects an element of $M$ also intersects an element of $N$.

ThEOREM 7.2. (1) If $N$ is a collection of point elements and either $M \in N$ 
or $N \supset M$, then $M<N$; (2) if $M$ is a collection of point elements and each element of $M$ is a portion of $N$, then $M<N$; (3) $\Sigma(N) \supset N$.

Proof. Consider (2). Let $E \in M$, and $F$ be a point element which intersects $E$. By Theorem 5.1 there exists a point element $G$ which is a lower bound of $E$ and $F$. Since $G<E<N, G<N$, by D 7.2 there exists $n \in N$ such that $G$ and $n$ intersect; since $G<F, n$ intersects $F$. By Theorem $7.1 M<N$. Clearly each element of $N$ is a portion of $N$; the conclusion of (1) of the theorem follows from (2).

The following theorem shows that the ordering relation $<$ is equivalent to the aggregate-inclusion relation $C$, if applied to the sets $\Sigma(X)$ and $\alpha(X)$; cf. $D$ 7.4. It brings out the importance of the atomic elements in the ordering theory.

Theorem 7.3. Let $M$ and $N$ each be point elements or collections of point elements: (1) $M<\Sigma(M)<\alpha(M)<M$; (2) the conditions (a) $M<N$, (b) $\Sigma(N)$ $\supset \Sigma(M)$, and (c) $\alpha(N) \supset \alpha(M)$ are logically equivalent; (3) the conditions (a) $M$ intersects $N$, (b) the product $\Sigma(M) \cdot \Sigma(N)$ is nonvacuous, and (c) the product $\alpha(M) \cdot \alpha(N)$ is nonvacuous are logically equivalent; (4) $\Sigma(M+N) \supset \Sigma(M)+\Sigma(N)$, and $\alpha(M+N)=\alpha(M)+\alpha(N)$; each of these four sets is equivalent in our ordering to $M+N$.

Condition (3) is an analogue of Theorem 5.1. Cf., also, Theorem 7.4. Condition (3) brings out the equivalence of D 7.1 and D 3.5.

Proof. Let the point element $E$ belong to $M$, and let $F$ be a point element which intersects $E$. By Theorems 5.1 and 5.5 there exists an atomic point element $G$ which is a lower bound of $E$ and $F$. By Theorem 7.2 (1), $E<M$; since $G<E, G<M$, and $G \in \alpha(M)$. By Theorem 7.1 $M<\alpha(M)$. Since $\Sigma(M) \supset \alpha(M)$, $\alpha(M)<\Sigma(M)$; cf. Theorem $7.2(1)$. By Theorem $7.2(2), \Sigma(M)<M$.

Let $M$ be a portion of $N$ and $X \in \Sigma(M)$; since $X<M<N, X<N$, and $X \in \Sigma(N)$; thus, $\Sigma(N) \supset \Sigma(M)$. Conversely, if $\Sigma(N) \supset \Sigma(M)$, it follows by part (1) and Theorem $7.2(1)$ that $M<\Sigma(M)<\Sigma(N)<N$. Thus (2a) and (2b) are equivalent. In the same way we can show that (2a) and (2c) are equivalent.

If $M$ and $N$ intersect, there exist intersecting point elements $E$ and $F$ which belong to $M$ and $N$, respectively (cf. D 7.1). By Theorems 5.1 and 5.5 there exists an atomic point element $G$ which is a lower bound of $E$ and $F$. By Theorem 7.2 (1), $E<M$ and $F<N$. It follows that $G<M$ and $G<N$, or, that $G \in \alpha(M) \cdot \alpha(N)$. Since $\Sigma(M) \cdot \Sigma(N) \supset \alpha(M) \cdot \alpha(N)$, (3a) implies each of (3b) and (3c). By D 7.1 and Theorem 5.1 each of these implies (3a).

By Theorem 7.2 (1), $\Sigma(M) \supset M$; the proof of part (4) follows with the help of this theorem and parts (1) and (2).

THEOREM 7.4. Let $M$ be a collection of point elements, and of aggregates of point elements: (1) The set of all the point elements which belong to elements of $M$ is a least upper bound of $M$; (2) if the elements of $M$ have lower a bound, the 
product of the sets $\alpha(m)$ and that of the sets $\Sigma(m)$, where $m$ ranges over $M$, are greatest lower bounds of $M$.

Proof. Let $K$ be the product of all the sets $\alpha(m)$ and $L$ that of all the sets $\Sigma(m)$. By our hypothesis and Theorem 5.5 neither $K$ nor $L$ is vacuous. By Theorem 7.2 (2), and Theorem 7.3 (1), each of $K$ and $L$ is a lower bound of the elements of $M$. Let $X$ be a lower bound of $M$, and $Y$ be an element of $M$. By Theorem $7.3 \alpha(Y) \supset \alpha(X)$; it follows that $K \supset \alpha(X)$. Since $L \supset K$, it follows from Theorems 7.2 and 7.3 that $X<\alpha(X)<K<L$. The conclusion of (2) follows. Part (1) may be proved by similar methods.

8. The $S$-portions, our ideal points. If $E$ is a nondegenerate point element and we remove a finite number of the elements of $E$, the remainder is a point element which is equivalent to $E$. Thus, the condition $\mathrm{P}_{2}$ for partially ordered systems does not hold for the quasi-partially ordered system of collections of point elements $A$, which we considered in the preceding section. In our applications to topology we find it desirable to remove the ambiguity which is involved in having equivalent, non-identical elements. We achieve this by a procedure which is essentially that of identifying equivalent elements; this yields a partially ordered system $\left({ }^{9}\right)$; its elements are our ideal points. For reasons given in $\$ 1$ we call these $S$-portions.

D 8.1. Let $A$ be the ordered system of all point elements and collections of point elements (cf. D 7.2 and D 7.3). If $E \in A$, let $P(E)$ be the collection of all elements of $A, X$, such that $X<E$ and $E<X$. We call $P(E)$ an $S$-portion or an ideal point. Clearly, $E \in P(E)$; if $X \in P(E)$, then $P(X)=P(E)$; for, if $Y \in P(X), E<X<Y$, and $Y<X<E$.

$D$ 8.2. Let $P$ and $Q$ be $S$-portions, $E \in P$, and $F \in Q$. If $E<F$ according to $\mathrm{D} 7.2$, we shall say that $P<Q$, or that $P$ is a portion of $Q$; also, we shall say that $P<F$ and $E<Q$.

D 8.3. Let $K(S)$ be the system of all $S$-portions; let it be ordered by the relation $P<Q$ of $\mathrm{D}$ 8.2. It is obvious that $K(S)$ is a partially ordered system; in particular, Condition $\mathrm{P}_{2}$ holds.

D 8.4. If one of the elements of an $S$-portion is a point element, the $S$-portion is called a composition point; if the point element is a boundary element or a decomposition point element, respectively, the point is called a boundary point or a decomposition point, respectively; cf. D 4.3. The theory of composition points corresponds to that of $\$ \S 4$ and 5 , while that of $S$-portions in general requires that of $\$ 7$. We shall see in $\$ 14$ that the composition points are included among the amalgamation points; these are a special class of the ideal points, and they are a basis for our topological applications. We shall see, also, that regular points and frontier points of $S$ may be identified with composition points; cf. Theorem 13.7.

EXAmples. E 8.1. Let $E$ be a finite collection of point elements such that

(') Cf. Birkhoff, p. 7, Theorem 1.2. 
if $E_{1}$ and $E_{2}$ are elements of $E$ then $a\left(E_{1}\right)=a\left(E_{2}\right)$; cf. D 4.2. Then $P(E)$ is a composition point. This follows from Theorems 14.11 and 14.8 ; or, it may be proved directly.

E 8.2. Let $P$ be a fixed point in the plane and $R$ be a ray with initial point $P$. Let $I(n, R)$ be $I-P$, where $I$ is the interval on $R$ with endpoint $P$ and length $1 / n$. Let $E(R)=(I(1, R), I(2, R), \cdots)$, and let $E$ be the set of all $E(R)$ 's. Let $R_{1}, R_{2}, R_{3}, \cdots$ be an infinite sequence of distinct $R$ 's. Let $F_{n}=I\left(n, R_{n}\right)+I\left(n+1, R_{n+1}\right)+\cdots$; let $F=\left(F_{1}, F_{2}, F_{3}, \cdots\right)$. Then $F$ is a point element but does not intersect any element of $E$; by Theorem $8.1 P(F)$ does not intersect $P(E)$, and not $P(F)<P(E)$. Suppose that $H$ is a point element and $H<E<H$; then each $E\left(R_{n}\right)$ intersects $H$, and $F$ intersects $H$; cf. D 4.4. Since $H<E, F$ intersects $E$, and we have a contradiction. Thus, $H$ does not exist, and $P(E)$ is not a composition point.

$E$ 8.3. If $E$ contains a boundary element and a decomposition point element, $P(E)$ is not a composition point.

$\mathrm{D}$ 8.5. If $M$ is a point or a point set let $\Sigma(M)$ and $\alpha(M)$ denote, respectively, all composition points and all atomic ideal points which are portions of $M$ (cf. D 7.4). Let $E_{P}$ denote an element of the ideal point $P$.

D 8.6. A degenerate composition point is one which has degenerate elements. (Cf. D 4.1.)

Theorem 8.1. Let $P$ and $Q$ be ideal points. (1) $\Sigma\left(E_{P}\right) \in P, \alpha\left(E_{P}\right) \in P$, and $\Sigma\left(E_{P}\right) \supset E_{P}$. (2) The following are equivalent: (a) $P<Q$; (b) $\Sigma\left(E_{Q}\right) \supset \Sigma\left(E_{P}\right)$; (c) $\alpha\left(E_{Q}\right) \supset \alpha\left(E_{P}\right)$. (3) The following are equivalent: (a) $P$ intersects $Q$; (b) $E_{P}$ intersects $E_{Q}$; (c) $\alpha\left(E_{P}\right) \cdot \alpha\left(E_{Q}\right)$ is nonvacuous. (4) If $P$ intersects $Q$, $P\left(\alpha\left(E_{P}\right) \cdot \alpha\left(E_{Q}\right)\right)$ is their greatest lower bound in $K(S)$.

(Cf. D 3.5 and the theorems of $\$ 7$.)

Theorem 8.2. An atomic $S$-portion is a composition point. A composition point is atomic if and only if its elements contain only atomic point elements.

Proof. Let $F \in E_{P}$ and $G \in \alpha(F)$; by Theorem $7.2 F<E_{P}$. Since $G<F<E_{P}$, $P(G)<P\left(E_{P}\right)=P$. If $P$ is atomic, $P=P(G), G \in P$, and $F<E_{P}<G$; since $G$ is atomic, so is $F$. The sufficiency may be proved by similar methods.

ThEOREM 8.3. Two atomic $S$-portions do not intersect.

Theorem 8.4. A degenerate $S$-portion is atomic.

The latter follows from Theorem 5.3. Let $P$ and $Q$ be atomic points which intersect in $X$. Then $X<P$; since $P$ is atomic, $P=X$; similarly, $Q=X$.

9. Intersections and orderings of collections of points. We extend the concepts of intersection and of order to the class of all points and all collections of points. Our definitions, methods, and results are similar to those of preceding sections. We introduce the concepts of the summation and the decomposi- 
tion of points. Example E 9.2 gives an indication of the complicated relations that may hold in our theory; some of the results in it may seem paradoxical.

$\mathrm{D}$ 9.0. The term, a real point is to mean a point of $S$. Hereafter, if the term point is used without any explicit or implicit qualifications, it may mean a real, an ideal, or a frontier point; cf. D 2.3.

$D$ 9.1. If a real point or a frontier point intersects $\left({ }^{10}\right)$ a point element which belongs to an element of an $S$-portion, the point and the $S$-portion are said to intersect. Two $S$-portions are said to intersect if they have a lower bound in the system of ideal points; cf. D 3.5. If a point intersects one of a collection of points, it and the collection are said to intersect; similarly define the intersection of two collections.

D 9.2. Let $B$ be the aggregate whose elements are the points and the collections of points. If $M$ and $N$ are elements of $B$ and each ideal point that intersects $M$ also intersects $N$, we say that $M$ is a portion of $N$, or that $M<N$. Clearly, this relation gives a quasi-partial ordering for $B$. The formulation of our definition is similar to the condition of Theorem 7.1. By Theorem 8.1 $K(S)$ is a subsystem of $B$ (cf. D 8.2 and $\mathrm{D} 8.3$ ).

D 9.3. If $P$ is a point and $M$ is a collection of points, $P<M$, and $M<P$, we say that $P$ is a summation of the elements of $M$; if, in addition, no two elements of $M$ intersect, $M$ is said to be a decomposition of $P . P(M)$ means an ideal point which is a summation of the elements of $M$.

D 9.4. If $M$ is the set of all point elements, $P(M)$ is the maximal $S$-portion. Clearly, the term is justified, and $P(M)$ is the summation of all composition points. It follows from Theorems 8.3 and 5.5 that the set of all atomic ideal points is a decomposition of $P(M)$.

EXAMPLES. E 9.1. Let $S$ be completely regular and $R$ be a perfectly compact Hausdorff space which is an immediate extension of $S$. Then $R$ is a decomposition of the maximal $S$-portion; cf. Theorem 16.1. Let $M$ and $N$ be the sets of all decomposition points and of all boundary points, respectively. Then $S<M$ and $M<S$; however, $M$ and $N$ fail to intersect. (Cf. Theorem 5.2.)

E 9.2. Let $S$ be the plane and $M$ be the set of all points of $S$. If $F$ is a closed set, let $E(F)$ be the set of all point elements $X$ such that each element of $X$ is a subset of $F$; let $Q(F)$ be the ideal point of which $E(F)$ is an element. Let $H$ be the aggregate of all $Q(L)$ 's, where $L$ is a line; and let $K$ be the set of all $Q(C)$ 's, where $C$ is the circumference of a circle with a positive radius. Since no point of $S$ is isolated, all point elements in $E(C)$ and in $E(L)$ are nondegenerate; it follows from D 4.1, Theorem 5.1 and $D 7.1$ that $E(C)$ and $E(L)$ do not intersect; by Theorem $8.1 Q(C)$ and $Q(L)$ do not intersect; by D 9.1 and D $9.2 H$ and $K$ do not intersect. Hence, neither $H<K$ nor $K<H$. If $P$ is a real point common to $C$ and $L$ it follows from D 4.5 that $P$ intersects elements of $E(C)$ and $E(L)$; it may be shown that $P$ intersects $I(C)$ and $I(L)$.

(10) Cf. D 4.5 . 
Since $I(C)$ intersects $L$ but does not intersect $I(L),{ }^{\circ} L$ is not a portion of $I(L)$; similarly, $C$ is not a portion of $I(C)$, and $M$ is a portion of neither $H$ nor $K$. By Theorem $10.3 E(L)$ contains boundary elements, but $E(C)$ does not; it follows that $Q(C)<C$, but not $Q(L)<L$; similarly, $K<M$, but not $H<M$.

Because of the similarity of the ideas involved in $\$ \S 7$ and 9 we can prove theorems analogous to those of $\$ 7$ for collections of points, and can use similar proofs. We give several theorems about summations and decompositions.

Theorem 9.1. If $P$ is an $S$-portion, $\alpha(P)$ is a decomposition of $P$, and $P$ is a summation of $\Sigma(P) ; P<\alpha(P)<\Sigma(P)<P$.

(2) If $M$ is a collection of ideal points, there exists one ideal point, $P(M)$, which is the summation of the elements of $M$; and $P(M)$ is the least upper bound of $M$ in the system of the ideal points.

Proof. By D $8.5 \Sigma(P) \supset \alpha(P)$ and each element of $\Sigma(P)$ is a portion of $P$. By the analogue of Theorem 7.2 for the case of points, $\alpha(P)<\Sigma(P)<P$. Let $X$ and $Y$ be ideal points such that $Y<X$ and $Y<P$. By Theorem 8.1 $\alpha\left(E_{P}\right) \cdot \alpha\left(E_{X}\right) \supset \alpha\left(E_{Y}\right)$. If $F \in \alpha\left(E_{Y}\right), F$ is an atomic point element; by Theorems 8.1 and $8.2 P(F)$ is atomic, $P(F)<X$, and $P(F)<P$. Hence $P(F) \in \alpha(P)$, and $X$ intersects an element of $\alpha(P)$. By D 9.2 $P<\alpha(P)$. By Theorem 8.3 $\alpha(P)$ is a decomposition of $P$. This completes the proof of (1).

Consider (2). Let $E$ be the sum of all sets $\alpha\left(E_{m}\right)$, where $m \in M$. Then $E$ is a collection of point elements; let $P=P(E)$. Since $E \supset \alpha\left(E_{m}\right)$, by Theorem 7.2 $\alpha\left(E_{m}\right)<E$; by Theorem 7.3 and D $8.2 m<P$; by the analogue of Theorem 7.2 for the case of points, $M<P$. By Theorem 8.1 if the ideal point $A$ intersects $P$ then $E_{A}$ intersects $E$. By Theorems 7.3 and $7.1 E_{A}$ intersects an element of $E$, $\lambda$; then $\lambda$ belongs to one of the sets, $\alpha\left(E_{m}\right)$, and $E_{A}$ intersects this set; by Theorem $8.1 A$ intersects $m$; by D $9.2 P<M$. Let the ideal point $Y$ be an upper bound of the elements of $M$. Then, for $m \in M, \alpha\left(E_{Y}\right) \supset \alpha\left(E_{m}\right)$ (cf. Theorem 8.1). Then $\alpha\left(E_{Y}\right) \supset E \supset \alpha\left(E_{m}\right)$. By Theorem 7.3 and D $8.2 m<P<Y$. Thus, $P$ is a summation of the elements of $M$ and is a least upper bound of them. Since the system of ideal points is partially ordered, $P$ is unique.

THEOREM 9.2. In the partially ordered system of ideal points each collection $M$ which has a lower bound has one greatest lower bound, $\beta_{M}$; and $\alpha\left(\beta_{M}\right)$ is the product of all the sets $\alpha(m)$, where $m \in M$.

Proof. Let $Y$ be an ideal point which is a lower bound of the elements of $M ; K$ be the product of all the sets $\alpha(m) ; \beta_{M}=P(K)$; and $m \in M$. If $\lambda \in \alpha(Y)$, then $\lambda<Y<m$, and $\lambda \in \alpha(m)$; thus $\alpha(m) \supset \alpha(Y)$, and $K \supset \alpha(Y)$. Let the ideal point $X$ intersect $Y$; by Theorem $9.1 Y<\alpha(Y)$, and by D $9.2 X$ intersects an element of $\alpha(Y)$; since this element belongs to $K$, it follows from $\mathrm{D} 9.2$ that $Y<\beta_{M}$. Similarly, we can prove that $\beta_{M}<m$. By Theorem 9.1 $K=\alpha\left(\beta_{M}\right)$. We have established the conclusion. 
10. Accessibility and ends. We develop a theory for the accessibility of $S$-portions; this involves an extension of the theory of $\S 6$, where we deal with point elements. The discussion and the examples which were given there are appropriate here. The ends may be regarded as barriers at the terminations of the corresponding ways. We borrow the term end from Carathéodory; in \$5 we indicate similarities between his treatment and ours. Theorem 11.3 brings out a dualism of the theories of the ends and of the $S$-neighborhoods; both are applied in the topologization of our ideal points, and in the development of this topology. The concepts of the present section, of overlapping and of being a common way, are analogous, respectively, to those of intersecting and of being a common portion; this may be seen by comparing the results of Theorem 10.1 with those of Theorems 5.1, 7.1, 7.4, and 9.2.

D 10.1. If $M$ is a set of real points, $K$ is a collection of point elements, and $M$ and some element of $K$ overlap ${ }^{(11)}$, we say that $M$ and $K$ overlap; also, $P(K)$ and $M$ overlap; if $P(K)$ is a portion of the real or the frontier point $Q$, we say that $M$ and $Q$ overlap.

D 10.2. If $P$ and $Q$ are points and each $S$-portion that intersects $P$ overlaps $M$ (and intersects $Q$ ), we say that $P$ is an end of $M$ (in $Q$ ). If $P$ and $Q$ are ideal points, $E \in P, F \in Q$, and $P$ is an end of $M$ in $Q$, we say that $E$ is a way to $P$ (in $F$ and in $M$ ) (cf. D 6.2). Clearly, the latter implies that $P<Q$.

Examples and comment. Adopt the notation of $E 4.1$; the composition point $P\left(E_{36}\right)$ is an end of the point set $e_{33}$ in the point $P\left(E_{12}\right)$ (cf. also E 6.2). Consider $\mathrm{E} 9.2$; the point $P(F)$ may be characterized as the summation of all composition points which are ends of $F$, and it is an end of $F ; E(F) \in P(F)$, and each point element of $E(F)$ consists of subsets of $F$; Theorem 10.1 shows that it is characteristic of an end of a closed set of $S$ that it have an element $E$ such that any point element which belongs to $E$ consists of subsets of the closed set. Such is not the case for all the elements of an end; for, consider $\mathrm{E} 4.3$; here $P(E)$ is an end of the $x$-axis, but no element of $E$ is a subset of the $x$-axis. If, in $\mathrm{E} \mathrm{9.2,} \mathrm{we} \mathrm{let} C^{\prime}$ and $L^{\prime}$ be the sums of all the $E(C)$ 's and of all the $E(L)$ 's, respectively, we have two ways in $S$ which have no common way; the ends of these ways, $P\left(C^{\prime}\right)$ and $P\left(L^{\prime}\right)$, do not intersect.

If $A$ is a point of a linear interval and $M$ is a countable sequence of points on the interval which converges to $A$, according to our theory there exist many more methods of approach to $A$ in the arc than there exist in the sequence. The end of the arc is to be regarded as vastly smaller than $A$, but is much greater than the end of the sequence; the latter is capable of further subdivision. Similar interpretations hold for the other examples.

TheOREM 10.1. Let $M$ be a closed point set in $S$ and $P$ be an $S$-portion. (1) In order that there exist an end of $M$ in $P$, it is necessary and sufficient that $M$ and $P$ should overlap. (2) Let $P$ and $M$ overlap, $G \in P$, and $G(M)$ be the collection of all

(i1) Cf. D 6.1. 
$E(M)$ 's $\left({ }^{12}\right)$, where $E$ is a point element which belongs to $G$; let $Q$ be the ideal point $P(G(M))$; then $Q$ is the maximal end of $M$ in $P$.

Proof. Let the ideal point $A$ be an end of $M$ in $P$. Since $A$ intersects itself, by $\mathrm{D} 10.2 A$ overlaps $M$; and $A<P$. Since $A$ overlaps $M$, there exists $E_{A} \in A$ which overlaps $M$ (D 10.1). Since $A<P$, by Theorem $8.1 \Sigma\left(E_{P}\right) \supset E_{A}$; by D $10.1 M$ overlaps each of $\Sigma\left(E_{P}\right)$ and $P$. The necessity of the condition in (1) follows.

Conversely, suppose that $P$ and $M$ overlap; by D 10.1 there exist $G \in P$ and a point element $X$, which belongs to $G$, such that $X$ overlaps $M$; by Theorem $6.1 X(M)$ is a point element; thus, $G(M)$ and $Q=P(G(M))$ exist. Let $Z$ be an ideal point which intersects $Q$. By Theorem $8.1 E_{Z}$ intersects $G(M)$; by $\mathrm{D} 7.1$ there exist point elements $E_{1}$ and $E(M)$ which belong to $E_{Z}$ and to $G(M)$, respectively, and which intersect. Since each element of $E(M)$ is a subset of $M$, it follows by $\mathrm{D} 4.4$ that each element of $E_{1}$ has points in common with $M$. By D 6.1 and D $10.1 M$ overlaps each of $E_{1}, E_{Z}$, and $Z$. There exists an element of $G, E$, such that each element of $E(M)$ is a subset of an element of $E$; then $E_{1}$ intersects $E$; by $\mathrm{D} 7.1 E_{Z}$ intersects $G$; by Theorem $8.1 Z$ intersects $P$. Since, also, $Z$ overlaps $M, Q$ is an end of $M$ in $P$ (cf. D 10.2).

Let $G_{1}$ be any element of $P$, whatever. Since each element of $E(M)$ is a subset of an element of $E$, it follows from D 4.4 and D 4.6 that $E(M)<E$; by Theorem $7.2 G(M)<G$, and thus $G(M)<G_{1}$; by Theorem $7.1 E(M)$ intersects an element, $K$, of $G_{1}$; since each element of $E(M)$ is a subset of $M$, it follows that $K$ overlaps $M$. By the results of the preceding paragraph it follows that $G_{1}(M)$ is nonvacuous; and, if $Q_{1}=P\left(G_{1}(M)\right)$, then $Q_{1}$ is an end of $M$ in $P$.

Let $\beta$ be an end of $M$ in $P$, and $Y$ be an ideal point which intersects $\beta$. Then $\beta<P$; by Theorem $8.1 \alpha(G) \supset \alpha\left(E_{\beta}\right)$, and $K=\alpha\left(E_{\beta}\right) \cdot \alpha\left(E_{Y}\right)$ is nonvacuous. Let $F \in K$; by Theorems 7.3 and $7.2 F<E_{\beta}$; by D $8.2 P(F)<\beta$; by D 10.2 $P(F)$ overlaps $M$; by the result of the preceding paragraph, $F$ overlaps $M$; by Theorem $6.1 F(M)$ exists; by arguments we have used before, $F(M)<F$ $<E_{\beta}<G$. By D $7.2 F(M)$ intersects an element $E$ of $G$. Since each element of $F(M)$ is a subset of $M$, it follows by an argument we used in the nextto-the-last paragraph that $E(M)$ exists and $F(M)$ and $E(M)$ intersect. Since $\alpha\left(E_{Y}\right) \supset K, F \in K$, and $F(M)<F$, it follows by Theorems 7.2 and 7.3 that $F(M)<F<K<E_{Y}$, and that $F(M) \in \Sigma\left(E_{Y}\right)$. Thus, $\Sigma\left(E_{Y}\right)$ and $G(M)$ intersect, since their respective elements $F(M)$ and $E(M)$ intersect. By Theorem 8.1 $Y$ and $Q$ intersect. Thus, by D $9.2 \beta<Q$. Since $\beta$ is an end of $M$ in $P$, $Q$ is the maximal end of $M$ in $P$. Since $Q_{1}<Q$, and $Q<Q_{1}, Q=Q_{1}$. Thus, $Q$ is independent of the particular element $G$ of $P$. We have established the truth of (2) and the sufficiency of the condition in (1).

Theorem 10.2. Let $M$ be a closed point set in $S, P$ be an end of $M$, and $Q$

${ }^{(2)}$ Cf. Theorem 6.1. For example, $E(M)$ is the set of all products $e \cdot M$, where $e \in E$. 
be an ideal point which intersects $P$; there exists an end of $M$ which is common to $P$ and $Q$. If $Q<P, Q$ is an end of $M$.

THEOREM 10.3. In order that the closed point set in $S, M$, be perfectly compact in itself, it is necessary and sufficient that no boundary point be an end of $M$.

Proof. Suppose that $M$ is not perfectly compact in itself; there exists in $M$ a monotonic collection of closed point sets, $K$, which have no point in common (cf. Moore, (II)). By D 4.1, $K$ is a boundary element. By Theorem 10.1, $P(K)$ is an end of $M$.

Conversely, let $M$ be perfectly compact in itself, and $E$ be a boundary element. Let $K$ be the aggregate of all products $e \cdot M$, where $e \in E$. If $E$ overlapped $M$, no element of $K$ would be vacuous; then the product of the elements of $K$ would be nonvacuous (cf. Fréchet, (I), p. 231). Then the product of the elements of $E$ would be nonvacuous; by D 4.1 this is impossible. Since $E$ does not overlap $M$, there exists no end of $M$ in $P(E)$ (cf. Theorem 10.1).

THEOREM 10.4. If the point set $M$ of $S$ is perfectly compact in itself and $P$ is a point and a limit point of $M$, there exists a composition point which is an end of $M$ in $P$.

Proof. If $R$ is a neighborhood of $P$ relative to $M$, let $e(R)=\bar{R}_{M}-P$. Let $E$ be the aggregate of all the $e(R)$ 's. Since $M$ is a closed point set in $S, \bar{R}_{M}$ is closed in $S$, and $e(R)$ is closed in $S-P$. Let $U$ be an open set in $S$ which contains $P$; then $U \cdot M$ is a neighborhood of $P$ relative to $M . M$ may be regarded as a regular subspace of $S$ (cf. Alexandroff and Hopf, p. 89, Theorem IX). It follows that $U \cdot M$ and $U$ contain an element of $E$. If we let $P=a(E)$, $E$ satisfies condition (4) of D 4.1. It may readily be verified that $E$ satisfies the remaining conditions. Thus, $E$ is a point element, and $Q=P(E)$ is a composition point. By Theorem $10.1 Q$ is an end of $M$.

Let the ideal point $X$ intersect $Q$; by $\mathrm{D} 10.2 X$ overlaps $M$. By Theorem $8.1 E_{X}$ intersects $E$; by $\mathrm{D} 7.1$ there exists a point element, $F$, which belongs to $E_{X}$ and intersects $E$. Since each $S$-neighborhood of $P$ contains an element of $E$, and each element of $F$ has points in common with each element of $E$, it follows that $F$ and $P$ intersect; cf. D 4.5. By D $9.1 X$ intersects $P$; by D 10.2 $Q$ is an end of $M$ in $P$.

11. The $S$-neighborhoods. These are applied in the topologization of our ideal points. The condition of "being separated by $S$-neighborhoods" of D 11.2 is analogous to the separations which are involved in Hausdorff's Axiom D and in the definition of normality; it finds analogous applications; cf. Theorem 11.5. The end theory of the preceding section is closely related to the $S$-neighborhood theory; duality of the two is brought out in Theorem 11.3. An important characterization of the atomic points is given in Theorem 11.7.

D 11.1. An open set in $S, D$, is called an $S$-neighborhood of $M$ if one of the following conditions holds: (1) $M$ is a point or a subset of $D$; (2) $M$ is a point 
element and $D$ contains an element of $M$; (3) $M$ is an ideal point, a collection of points, or an aggregate of point elements, and $D$ is an $S$-neighborhood of each element of $M$. Thus, to see whether $D$ is an $S$-neighborhood of the $S$-portion $P$, first apply (2) for each point element which belongs to some element of $\mathrm{P}$, then apply (3) for each element of $P$; then apply (3) for $P$. (Cf. D 2.3.)

D 11.2. If $U$ and $V$ are mutually exclusive open sets in $S$ which are $S$-neighborhoods of $M$ and of $N$, respectively, we say that they separate $M$ and $N$ (relative to $S$ ).

THEOREM 11.1. If $K$ is an $S$-portion or a collection of point elements, and $D$ is an open set in $S$, then $D$ is an $S$-neighborhood of $K$ if and only if $K$ and $S-D$ do not overlap.

Proof. Let $D$ be an $S$-neighborhood of the $S$-portion $P, E \in P$, and $\lambda \in E$. By D $11.1 D$ is an $S$-neighborhood of $\lambda$ and of $E$; and there exists $\beta \in \lambda$ such that $D \supset \beta$. Then $\beta \cdot(S-D)$ is vacuous; by $\mathrm{D} 6.1$, Theorem 10.1 , and $\mathrm{D} 10.1$, neither $\lambda, E$, nor $P$ overlaps $M$. The converse may be established by reversing this argument.

THEOREM 11.2. If $E$ is an element of the ideal point $P$, then $E$ and $P$ have the same $S$-neighborhoods.

Proof. Let $D$ be an open set in $S$. By Theorem 10.1 if some element of $P$ overlaps $S-D$, so does every other element. The conclusion follows from Theorem 11.1 and D 11.1.

Theorem 11.3. Let $D$ be an open set in $S$ and $P$ be an ideal point. (1) In order that $D$ be an $S$-neighborhood of $P$ it is necessary and sufficient that $S-D$ have no end in $P$; (2) in order that $P$ be an end of $S-D$ it is necessary and suffcient that $D$ fail to be an $S$-neighborhood of any portion of $P$.

Proof. If $D$ is an $S$-neighborhood of $P$, by Theorem $11.1 P$ does not overlap $S-D$. By Theorem 10.1, $S-D$ has no end in $P$. A similar argument proves the converse.

Let $P$ be an end of $S-D$ and $X$ be a portion of $P$. Since $X$ intersects $P$, by D $10.2 X$ overlaps $S-D$; by Theorem $11.1 D$ is not an $S$-neighborhood of $X$. Conversely, suppose that $D$ is not an $S$-neighborhood of any portion of $P$. Let $Z$ be an ideal point which intersects $P$. By Theorem $8.1 P\left(\alpha\left(E_{P}\right) \cdot \alpha\left(E_{Z}\right)\right)$ is a lower bound of $P$ and $Z$. By our hypothesis and Theorem $11.1 S-D$ overlaps $P\left(\alpha\left(E_{P}\right) \cdot \alpha\left(E_{Z}\right)\right)$; by Theorem 10.1 and D $10.1 S-D$ overlaps $\alpha\left(E_{P}\right) \cdot \alpha\left(E_{Z}\right), \alpha\left(E_{Z}\right)$, and $Z$. By D $10.2 P$ is an end of $S-D$.

THEOREM 11.4. Let $D$ be an $S$-neighborhood of the ideal point $X$, and $P$ be a non-isolated point of $D$; then $D-P$ is an $S$-neighborhood of $X$.

Proof. If $E$ is a point element which belongs to an element of $X$, by $\mathrm{D} 11.1$ there exists $e_{1} \in E$ such that $D \supset e_{1}$. If $a(E)=P$, it follows from the fact that $P$ 
is non-isolated that $S-P \supset e_{1}$ (cf. D 4.1 and D 4.2). If $a(E) \neq P$ there exist elements of $E, e_{2}$ and $e$, such that $S-P \supset e_{2}$ and $e_{1} \cdot e_{2} \supset e$ (cf. D 4.1). Then $D-P \supset e$. By D $11.1 D-P$ is an $S$-neighborhood of $E$ and of $X$.

THEOREM 11.5. In order that a real or a frontier point should fail to intersect a composition point, it is necessary and sufficient that the two points be separated by a pair of their respective S-neighborhoods.

Proof. Let the real point $P$ be separated from the composition point $Q$ by $U$ and $V$. Then by D $11.1 V$ is an $S$-neighborhood of any point element which belongs to an element of $Q$; by D 4.5 and D $9.1 P$ intersects neither the point element nor $Q$. Conversely, suppose that $P$ and $Q$ do not intersect, and let the point element $E$ be an element of $Q$. By D 9.1 $P$ and $E$ do not intersect; by D $4.5 P$ and $E$ can be separated by a pair of their respective $S$-neighborhoods, $U$ and $V$; by Theorem $11.2 \mathrm{~V}$ is an $S$-neighborhood of the point $Q$.

THEOREM 11.6. If each of $M$ and $N$ is an ideal point or a collection of ideal points and $M<N$, then each $S$-neighborhood of $N$ is an $S$-neighborhood of $M$.

Proof. Suppose that $D$ is an $S$-neighborhood of $N$ but not of $M$; there exists $P \in M$ such that $D$ is not an $S$-neighborhood of $P$. By Theorem 11.3 $S-D$ has an end in $P$, say $Q$; then $Q<P$. Since $Q$ intersects $P$ and $M<N$, by D $9.2 Q$ intersects an element $Z$ of $N$; let $\beta$ be a lower bound of $Q$ and $Z$. If $\lambda \in \beta, \Sigma\left(E_{Z}\right) \supset \lambda$ and $\Sigma\left(E_{Z}\right) \in Z$ (cf. Theorem 8.1). Since $Z \in N, D$ is an $S$-neighborhood of $Z$, of $\Sigma\left(E_{Z}\right)$ and of $\lambda$ (cf. D 11.1). Since $\lambda$ is an arbitrary element of $\beta, D$ is an $S$-neighborhood of $\beta$; since $\beta<Q$, by Theorem 11.3, (2), we are involved in a contradiction. Thus, $D$ does not exist.

THEOREM 11.7. An S-portion $P$ is atomic if and only if it satisfies the following condition: If the sum of a finite number of open sets in $S$ is an $S$-neighborhood of $P$, at least one of them is an $S$-neighborhood of $P$.

If $P$ is nondegenerate, the following is an equivalent condition : If $M$ and $N$ are closed point sets in $S$ and have at most a finite number of common points, then $P$ is not an end of both $M$ and $N$.

Proof. Let $D_{1}$ and $D_{2}$ be open sets whose sum $D$ is an $S$-neighborhood of $P$. Let $S-D_{1}=M_{1}$ and $S-D_{2}=M_{2}$. Suppose that neither $D_{1}$ nor $D_{2}$ is an $S$-neighborhood of $P$; by Theorem $11.3 M_{1}$ and $M_{2}$ have ends in $P$. If $P$ is atomic, these ends are $P$. Let $E$ be a point element such that $E \in P$. There exists $e \in E$ such that $D \supset e$. By Theorems 6.1 and $10.1 E\left(M_{1}\right)$ and $E\left(M_{2}\right)$ are point elements and are elements of $P$. Then $e \cdot M_{1} \in E\left(M_{1}\right)$ and $e \cdot M_{2} \in E\left(M_{2}\right)$. Since any two elements of $P$ intersect, by D $4.4\left(e \cdot M_{1}\right) \cdot\left(e \cdot M_{2}\right)$ is nonvacuous. Since $D \supset e$ and $M_{1} \cdot M_{2}=S-D$, we have a contradiction. Thus, the condition is necessary.

Suppose that $P$ is not atomic and $D$ is an $S$-neighborhood of $P$. By Theo- 
rems 8.1, 8.2, and 8.3 there exist two atomic point elements, $E$ and $F$, which belong to $\alpha\left(E_{P}\right)$ but do not intersect. By D 4.4 and D 11.1 there exist $e \in E$ and $f \in F$ such that $D \supset e+f$ and $e \cdot f$ is vacuous. Let $D_{1}=D-\bar{e}$ and $D_{2}=D-\bar{f}$. Since $D_{1}$ does not contain $e$, it is not an $S$-neighborhood of $E$ or of $P$; similarly, $D_{2}$ is not an $S$-neighborhood of $P$. By D 4.1 and D 4.2 if $a(E) \neq a(F)$, then $\bar{e} \cdot \bar{f}$ is vacuous and $D_{1}+D_{2}=D$. By $\mathrm{D} 4.1$ if $a(E)=a(F)$, then $e+a(F)=\bar{e}$, $f+a(F)=\bar{f}$, and $\bar{e} \cdot \bar{f}=a(F)$; since $e \cdot f$ is vacuous, $a(F)$ is non-isolated. Then $D_{1}+D_{2}=D-a(F)$, and by Theorem 11.4 this sum is an $S$-neighborhood of $P$. Thus, the condition is sufficient.

\section{Chapter II. Topological properties of the ideal points}

In the first chapter we considered intersection and order; our treatment now requires the introduction of relations which involve continuity. In $\$ 12$ we introduce a topology with the help of the $S$-neighborhoods. As a suggestion of problems we shall consider and of methods we shall use, we refer the reader to Alexandroff and Hopf, loc. cit., pp. 95-98; note particularly Theorem VIII. In this theorem they consider a perfectly compact Hausdorff space $X$, a decomposition $\sum A$ of $X$ into mutually exclusive closed point sets, and a mapping $y=\alpha(x)$ of $X$ into a space $Y$ such that $\alpha\left(x_{1}\right)=\alpha\left(x_{2}\right)$ if and only if $x_{1}$ and $x_{2}$ are elements of the same element of the decomposition. They develop equivalent conditions, which include: (1) the transformation is continuous and $Y$ is a perfectly compact Hausdorff space; (2) the transformation is continuous, and $Y$ is a Hausdorff space; (3) the decomposition is upper semi-continuous.

In our Theorems $15.3,15.4$ and 15.5 we obtain extensive generalizations of the results of these authors. These results involve properties which restrict our attention to a special class of the $S$-portions, the amalgamation points. In $\$ 14$ we develop a geometric theory of these points in which the amalgamation points have properties similar to those of the perfectly compact point sets in Alexandroff and Hopf's treatment. In Theorem 15.5 this analogy is strengthened by a kind of representation theory $\left({ }^{13}\right)$ in which the amalgamation points are identified with certain perfectly compact collections of amalgamation points, to which Alexandroff and Hopf's results may be applied. Corresponding to the set-theoretic treatment of upper semi-continuity of these authors our treatment requires the introduction of a geometric upper semicontinuity theory; cf. D 15.1. The latter part of the paper is devoted to the application of the basic theorems of $\$ 15$.

In $\$ 13$ we give characterizations of various types of regular and normal spaces in terms of the regular composition points. In $\$ \$ 16$ and 17 we find that these characterizations prove useful in applying the results of $\$ 15$. Such applications are particularly simple and extensive for the case of the semi-completely normal spaces, which we introduce in $\$ 13$. For them the development

(13) Cf. Birkhoff, p. 76. Our representations involve not merely order but also continuity. 
of Chapter II has a completeness that is attained for more general spaces only in Chapter III.

12. The topology of our points. We first give the following definition.

D 12.1. If $P$ is a point and $M$ is a point set, and each $S$-neighborhood of $P$ is an $S$-neighborhood of infinitely many elements of $M$, then $P$ is a limit point of $M$. Similarly, if $P$ and $M$ are a point element and a collection of point elements, respectively, $P$ is called a limit element of $M$.

TheOREM 12.1. The space $T(M)$, which is obtained by topologizing $M$ by $\mathrm{D} 12.1$ is a space $H$ Fréchet. Let $U$ be an open set in $S$ and let the symbol $U(M)$ denote any nonvacuous subset of $M$ consisting of all elements of $M$, with the possible exception of a finite number, of which $U$ is an $S$-neighborhood. The collection of all such $U(M)$ 's may be taken as a system of neighborhoods for defining limit points in $T(M)$.

If $P$ is the point and $N$ is a subset of $M$, clearly $P$ is a limit of $N$ according to $\mathrm{D} 12.1$ if and only if each $U(M)$ that contains $P$ contains points of $N$ distinct from $P$. By Fréchet, (I), pp. $185-186, T(M)$ is a space $H$.

THEOREM 12.2. If the composition point $P$ neither intersects nor is a limit point of the set $M$ of composition points, there exists an $S$-neighborhood of $P$ which is not an S-neighborhood of any element of $M$.

Proof. $P$ has an $S$-neighborhood $D$, which is an $S$-neighborhood of at most a finite number of elements of $M, X_{1}, X_{2}, \cdots, X_{m}$. Let $E$ and $F_{i}$ be point elements which are elements of $P$ and of $X_{i}$, respectively. There exist $e_{i} \in E$ and $f_{i} \in F_{i}$ such that $e_{i} \cdot f_{i}$ is vacuous. By D 4.1 there exists $e \in E$ such that $e_{1} \cdot e_{2} \cdot e_{3} \cdots e_{m} \supset e$. Let $U=\left(S-\bar{f}_{1}\right) \cdot\left(S-\bar{f}_{2}\right) \cdots\left(S-\bar{f}_{m}\right)$. Then $U \supset e$ and $U$ is an $S$-neighborhood of $E$ and of $P$; but $U$ is not an $S$-neighborhood of $F_{i}$ or of $X_{i}$. Then $D \cdot U$ satisfies the conclusion.

THEOREM 12.3. In order that a composition point be a limit point of a closed set of $S$, it is necessary and sufficient that the point be nondegenerate and contain an end of the set.

Proof. Let $M$ be the set, $P$ be a point which satisfies the condition, and $E$ be a point element which is an element of $P$. By Theorem $10.1 P$ and $E$ overlap $M$; by $\mathrm{D} 6.1$ each element of $E$ contains points of $M$. By the hypothesis and D 8.6 $E$ is nondegenerate; by D 4.1 no point of $M$ is common to all elements of $E$; thus, each of these elements contains infinitely many points of $M$; by D 11.1 each $S$-neighborhood of $P$ contains an element of $E$. Thus, $P$ is a limit point of $M$.

If there exists no end of $M$ in $P$, by Theorem $11.3 S-M$ is an $S$-neighborhood of $P$. Then $P$ is not a limit point of $M$. If $P$ is degenerate by $\mathrm{D} 8.6$ and D $4.1 a(E)$ is an isolated point of $S$; then $a(E)$ is an $S$-neighborhood of $E$ and of $P$, and $P$ is not a limit point of $M$. 
D 12.2. If $M$ is a collection of point elements, let $a(M)$ be the collection of all real points which are attached to elements of $M$ (cf. D 4.2). If $M$ is a real point or a frontier point, let $a(M)$ be $M$ or the null set, respectively. If $E$ is a set of points, or is an ideal point, let $a(M)$ be the sum of all sets $a(m)$, where $m \in M$. The concept $a(M)$ is applied in the definition of regularity (cf. D 13.1). In Example E 9.2, $a(Q(F))=F$. The method of the definition involves an induction similar to that of $\mathrm{D} 11.1$.

Theorem 12.4. Let $P$ and $Q$ be ideal points, $P<Q, E \in P$, and $D$ be an $S$-neighborhood of $P$. Then $a(Q) \supset a(P), a(P)=a(E)$, and $\bar{D}_{S} \supset a(P)$.

Proof. By D 8.2 if $F \in Q, E<F$. If $X \in a(E)$, there exists $\lambda \in E$ such that $a(\lambda)=X$. By Theorem 7.1 there exists $\delta \in F$ such that $\lambda$ intersects $\delta$; by Theorem $5.1 a(\lambda)=a(\delta)$. Thus, $a(F) \supset a(E)$. Since $a(Q)=\sum a(F)$, for $F \in Q$, and $a(P)=\sum a(E)$, for $E \in P$, it follows that $a(Q) \supset a(P)$. If $P=Q$, then $E<F$, and $F<E$; then $a(E) \supset a(F)$, and $a(F) \supset a(E)$; thus, $a(E)=a(F)$; since $F$ is any element of $Q=P, a(E)=a(P)$. By $\mathrm{D} 11.1 D$ is an $S$-neighborhood of $\lambda \in E \in P$. If $X \in S-\bar{D}_{S}$, by D 4.1 and D $12.2 X$ is not an element of $a(\lambda)$, of $a(E)$, or of $a(P)$.

13. Regular points and normal spaces. Since we deal extensively with perfectly compact Hausdorff spaces of our ideal points, we find need of the concept "the regularity relative to $S$ of an ideal point." In terms of this concept we characterize various types of regular and of normal spaces (Theorems 13.1 to 13.3). These characterizations are of interest, since the regularity of a Hausdorff space does not imply its normality. These characterizations yield methods for applications of the decomposition and mapping theory of $\S 15$; the most extensive results are given for the semi-completely normal spaces, which we introduce in this section. Normality proves to be a weaker condition than semi-complete normality; and the latter is weaker than complete normality. In Theorem 13.7 we show that regular real or frontier points may be replaced by regular composition points in questions which involve either continuity or order. This procedure gives a basis for applying our theory to questions concerning the embedding of $S$ in other spaces, and to those involving mappings of $S$. In general our methods do not require the regularity or the normality of the basic space; they are applicable locally, so long as they deal with regular ideal points. For instance, we are in a position to study an infinitesimal regular portion of an irregular point. For the plane all composition points are regular.

D 13.1. A point $P$ is regular (relative to $S$ ) provided that if $D$ is an $S$-neighborhood of $P$ there exists an $S$-neighborhood of $P, R$, such that $D+a(P) \supset \bar{R}_{S}$ (cf. D 12.2 and Alexandroff and Hopf, p. 68).

D 13.2. $S$ is said to be semi-completely normal provided that if $P$ is a point of $S$, then the subspace of $S, S-P$, is normal.

Clearly each completely normal space has this property; the plane is an 
example. In Theorem 13.4 we show that each semi-completely normal space is normal; an example by Tietze shows that the converse is not true (loc. cit., pp. 304-306). An example by F. B. Jones shows that if the hypothesis of the continuum is valid, then there exists a semi-completely normal space which is not completely normal. He has constructed a space which is normal (loc. cit., pp. 673-675). On page 674 he has a point set $N$, which is uncountable and contains no limit point of itself. By his Theorem 4 and the continuum hypothesis $S$ is not completely normal. It is obvious from his construction of $S$ that if $P$ is a point of $S$ then $S-P$ is normal.

THEOREM 13.1. (1) In order that a point of $S$ be regular, it is necessary and sufficient that it intersect no boundary point. (2) An irregular point of $S$ intersects an irregular boundary point.

Thus, a regular space is characterized by the property that it be a Hausdorff space and that none of its points intersect a boundary point.

Proof. Let $P$ be irregular, and $U$ be an open set containing $P$ such that if $R$ is an $S$-neighborhood of $P$, then $\bar{R} \cdot(S-U)$ is nonvacuous. If $M=\bar{U}-U$ were perfectly compact, it would be possible to separate $P$ and $M$ by a pair of open sets, $U_{1}$ and $U_{2}\left({ }^{14}\right)$; then $U \cdot U_{1}$ is an open set which contains $P$ and is a subset of $U$; the closure of this set is a subset of $U$, since it contains no point of $U_{2}$ and $U_{2} \supset M$. Thus we are involved in a contradiction; it follows that there exists a collection of open sets, $G$, which covers $M$ such that no finite subcollection of $G$ covers $M$ (cf. Kuratowski and Sierpiński, loc. cit.). For $X \in M$ let $U(X)$ and $W(X)$ be a definite pair of mutually exclusive open sets which contain $X$ and $P$, respectively, such that $U(X)$ is a subset of some element of $G$. Clearly, no finite collection of the $U(X)$ 's covers $M$. Let $D$ denote the sum of a finite collection of the $U(X)$ 's, $F=M-M \cdot D$, and $E$ be the set of all $F$ 's. It follows that $E$ is a boundary element; let it be an element of the boundary point $\beta$. Suppose that $\beta$ is regular; by Theorem $11.4 S-P$ is an $S$-neighborhood of $\beta$; since $a(\beta)$ is vacuous, by $\mathrm{D} 13.1$ there exists an $S$ neighborhood of $\beta, V$, such that $S-P \supset \bar{V}$; then $S-\bar{V}$ is an open set containing $P$. Since $V$ contains an element of $E, M-M \cdot V$ can be covered by a finite collection of the $U(X)$ 's, say $U\left(X_{1}\right), U\left(X_{2}\right), \cdots, U\left(X_{n}\right)$. The sum of $V$ and this finite collection is an open set which contains $M$. Let $\delta=U \cdot(S-\bar{V}) \cdot W\left(X_{1}\right) \cdots W\left(X_{n}\right)$. Then $\delta$ is an open set which contains $P$, $\delta \cdot M$ is vacuous, and $U \supset \bar{\delta}$. Thus, the supposition that $\beta$ is regular involves a contradiction. Suppose that $P$ and $\beta$ did not intersect; then by Theorem 11.5 $\beta$ and $P$ can be separated by a pair of their respective $S$-neighborhoods, $V$ and $W_{1}$; by Theorem 11.2 and $\mathrm{D} 11.1 V$ contains an element of $E$; since $\bar{V}$ does not contain $P$ we arrive at a contradiction as we did above. Thus, we have established the truth of (2) and the sufficiency of the condition in (1).

(14) Follow the proof of Theorem IX, p. 89, Alexandroff and Hopf. 
Let $P$ be regular, $E$ be a boundary element, and $\beta=P(E)$. By D 4.1 there exists $e \in E$ such that $P \in S-e$. Since $e$ is closed in $S$, there exists an open set $U$, containing $P$ such that $S-e \supset \bar{U}$. By D 11.1 and Theorem $11.2 S-\bar{U}$ is an $S$-neighborhood of $E$ and of $\beta$. By Theorem $11.5 P$ and $\beta$ do not intersect. Thus, the condition is necessary.

TheOREM 13.2. A Hausdorff space is normal if and only if each of its boundary points is regular.

Proof. Let $S$ be normal, $E$ be a boundary element, and $D$ be an $S$-neighborhood of the boundary point $P(E)$. By D 11.1 there exists $e \in E$ such that $D \supset e$. Since $S-D$ and $e$ are closed and mutually exclusive, they can be separated by a pair of mutually exclusive open sets $U$ and $V$. Since $V \supset e$, by Theorem $11.2 V$ is an $S$-neighborhood of $P(E)$; also $D \supset \bar{V}$.

Conversely, let the condition hold in $S$ and $M$ and $N$ be mutually exclusive closed sets. By Theorem 13.1 each point of $S$ is regular. For $P \in M$ let $D(P)$ be an open set which contains $P$ such that $N \cdot \overline{D(P)}$ is vacuous. Let $D$ denote any open set which is the sum of a finite number of the $D(P)$ 's; and let $E$ be the aggregate of all the sets $M-M \cdot D$. If one of the $D$ 's contains $M$, the complement of its closure contains $N$; if not, $E$ is a boundary element. Since $S-N \supset M, S-N$ is an $S$-neighborhood of $P(E)$; by our condition there exists an $S$-neighborhood of $P(E), U$, such that $S-N \supset \bar{U}$; then $U$ contains an element of $E$, and there exists a $D$ such that $U \supset M-M \cdot D$. Then $D+U \supset M$, and $N \cdot(\bar{D}+\bar{U})$ is vacuous. Thus, the complement of $\bar{D}+\bar{U}$ is an open set which contains $N$, and the condition is sufficient.

TheOREM 13.3. A Hausdorff space is semi-completely normal if and only if each of its composition points is regular.

Proof. Let $S$ be semi-completely normal, $\beta$ be a composition point, and $E$ be a point element which is an element of $\beta$. If $\beta$ is degenerate, then $a(\beta)$ is an isolated point of $S$ and is an $S$-neighborhood of $\beta$; then $\beta$ is regular (cf. $\mathrm{D} 8.6$ and $\mathrm{D} 4.1$ ). If $a(\beta)$ is a non-isolated point of $S$, then by $\mathrm{D} 13.2$ the subspace $S-a(\beta)$ of $S$ is normal; then $\beta$ is a boundary point of this subspace; by Theorem $13.2 \beta$ is a regular boundary point of this subspace. It follows with the help of Theorem 11.4 that $\beta$ is a regular decomposition point of $S$. If $\beta$ is a boundary point of $S$ let $E$ be a point element, $E \in \beta$; let $P$ be a point of $S$; and let $F$ be the collection of all elements of $E$ which do not contain $P$; it follows that $F$ is a boundary element of $S$ and of $S-P$; also, in $S, F<E$, $E<F$, and $F \in \beta$; by the argument we used above it follows that $F$ is an element of a regular boundary point of $S-P$; and, hence, that $\beta$ is regular relative to $S$.

Conversely, let all composition points of $S$ be regular and let $P$ be a point of $S$. By Theorem 13.2 $S$ is normal and $S-P$ is regular. Methods analogous to those used for Theorem 13.2 show that $S-P$ is normal; by D $13.2 S$ is semi-completely normal. 
TheOREM 13.4. A semi-completely normal space is normal.

By Theorem 13.3 all the boundary points are regular; the conclusion follows from Theorem 13.2.

THEOREM 13.5. If $F$ is a finite point set in a semi-completely normal space $S$, then $S-F$ is semi-completely normal.

This may be proved with the help of Theorem 13.3 and the methods used in establishing that theorem.

TheORem 13.6. Let a regular point $P$ be added to $S$ so that the space $S+P$ is a Hausdorff space. If $S$ is normal, so is $S+P$; in order that $S+P$ be semicompletely normal, it is necessary and sufficient that $S$ have the same property.

Thus a semi-completely normal space is characterized by the following property: If we either add or remove a finite number of regular points, or do both, and the result is a Hausdorff space, it is normal.

Proof. Let $Q$ be a boundary point of the space $S+P$. Since $P$ is a regular point, by Theorem 13.1 $Q$ and $P$ do not intersect relative to $S+P$; by Theorem 11.5 there exist in $S+P$ mutually exclusive open sets $U$ and $V$ which are $(S+P)$-neighborhoods of $Q$ and $P$, respectively. Let $E$ be a point element of $S+P$ such that no element of $E$ contains $P$ and that $E \in Q$; then $E$ is a point element of $S$. Since $S$ is normal and $U$ is also an $S$-neighborhood of $E$, it follows from Theorem 13.2 that there exists an $S$-neighborhood of $E$, $W$, such that $U \supset \bar{W}$. Since the closures of $W$ in $S$ and in $S+P$ are identical, it follows that $Q$ is a regular boundary point of $S+P$. By Theorem 13.2 $S+P$ is normal.

TheOREM 13.7. Let $P$ be a real, a frontier, or a composition point which is regular; if $a(P)$ is a non-isolated point of $S$, let $F(P)$ be the aggregate of all sets, $\bar{D}_{S}-a(P)$, where $D$ is an $S$-neighborhood of $P$; otherwise, let $F(P)$ be the set of all the $\bar{D}_{S}$ 's. Let $Q(P)$ be the composition point of which $F(P)$ is an element. Then (1) $a(P)=a(Q(P)), P<Q(P)$, and $Q(P)<P$; (2) if $P$ is a limit point of a point set, then so is $Q(P)$; and conversely; (3) each $S$-neighborhood of $P$ is an $S$-neighborhood of $Q(P)$; if $D$ is an $S$-neighborhood of $Q(P)$, either $D$ or $D+a(P)$ is an $S$-neighborhood of $P ;(4) Q(P)$ is regular.

Thus, $Q(P)$ is equivalent to $P$ both from the point of view of order and of continuity. The topological applications of our theory require such a twofold equivalence; we discuss this in the introductions to Chapter II and to $\$ \$ 14$ and 15.

Proof. Let $P$ be a composition point and $E$ be a point element such that $E \in P$. Since each element of $F(P)$ contains an element of $E, E<F(P)$ (cf. D 4.6). Let $H$ be a point element which does not intersect $E$; there exist $e \in E$ and $h \in H$ such that $e \cdot h$ is vacuous. Then $S-\bar{h} \supset e$; by Theorem $11.2 S-\bar{h}$ 
is an $S$-neighborhood of $P$. By D 13.1 there exists an $S$-neighborhood of $P$, $U$, such that $a(P)+(S-\bar{h}) \supset \bar{U}_{S}$. Then $\bar{U}_{S}-a(P)$ or $\bar{U}_{S}$ is an element of $F(P)$, and contains no point of $h$. By D $4.4 F(P)$ and $H$ do not intersect. (It may readily be verified by $\mathrm{D} 4.1$ that $F(P)$ is a point element.) It follows that $F(P)<E$ (cf. D 4.6). Since also $E<F(P)$, it follows by D 8.2 that $P<Q(P)$ and $Q(P)<P$; thus, $P=Q(P)$.

Next, let $P$ be a real point or a frontier point. Clearly, $F(P)$ satisfies the conditions of $\mathrm{D} 4.1$ and is a point element. Let $X$ be a composition point which does not intersect $P$. By Theorem $11.5 P$ and $X$ can be separated by a pair of their respective $S$-neighborhoods, $U$ and $V$. There exists an $S$-neighborhood of $P$, $W$, such that $U \supset \bar{W}_{S}$. Then $U$ contains an element of $F(P)$. By D $11.1 \mathrm{~V}$ contains elements of each point element which belongs to $X$; by $\mathrm{D} 4.4, \mathrm{D} 7.1$, and Theorem 8.1 $Q(P)$ and $X$ do not intersect. By D $9.2 Q(P)<P$. Conversely, let $X$ be an ideal point which does not intersect $Q(P)$; by Theorem 8.1 , if $G$ is a point element belonging to $X, G$ does not intersect $F(P)$. By D 4.4 there exist $f \in F(P)$ and $g \in G$ such that $f \cdot g$ is vacuous. There exists an $S$-neighborhood of $P, D$, such that either $f=\bar{D}_{S}$ or $f=\bar{D}_{S}-P$. By D $4.1 S-\bar{D}_{S}$ contains $g$ and is an $S$-neighborhood of $G$. By D 4.5 and D $9.1 P$ intersects neither $G$ nor $X$. By D $9.2 P<Q(P)$.

Since $P$ is regular, each of its $S$-neighborhoods contains an element of $F(P)$ and is an $S$-neighborhood of $Q(P)$. If $D$ is an $S$-neighborhood of $Q(P)$, $D \supset f \in F(P)$ (D 11.1 and Theorem 11.2). Then $f+a(P)=\bar{R}_{S}$, where $R$ is an $S$-neighborhood of $P$ and of $Q(P)$. Then $D+a(Q(P)) \supset \bar{R}_{S} \supset R$, and $Q(P)$ is regular; since $a(Q(P))=a(P)$, either $D+a(P)$ or $D$ is an open set containing $R$, and is an $S$-neighborhood of $P$.

If $P$ is a non-isolated point of $S$, the conclusion of (2) follows from (3), (4), D 12.1, and Theorem 11.4. For the other cases, $P$ and $Q(P)$ have the same $S$-neighborhoods, and (2) follows.

14. The amalgamation points. Suppose that the regular space $T$ is an immediate extension of $S$. By Theorem 13.7 we can replace each point of $S$ by an equivalent decomposition point, and each point of $T-S$ by a boundary point, and thus obtain a space $T_{1}$ of composition points which is topologically equivalent to $T$. We now introduce the amalgamation points in order to make more general applications, such as those we discuss in the introductions of Chapter II and of $\S 13$. These applications require that the amalgamation points include the composition points and that they have special properties which do not hold for all ideal points. These properties are similar to those of closed point sets in perfectly compact Hausdorff spaces. Thus, in Theorems $14.3,14.4$, and 14.5 we establish for amalgamation points a property analogous to the Borel-Lebesgue covering property; this analogy is emphasized in Theorem 15.3. Theorem 14.11 corresponds to the theorem that a nonvacuous product of perfectly compact sets is perfectly compact. In Theorems 14.6 and 14.7 we establish a condition for the separation of non-intersecting amalgama- 
tion points; this suggests the condition for the separation of closed sets, which characterizes the normality of a Hausdorff space.

In Theorems 14.12 and 14.13 we add to the characterizations of the normal and the semi-completely normal spaces. For such spaces we obtain an extensive knowledge concerning the atomic elements of the developments of $\S \S 15$ and 16 . For the Hausdorff spaces in general such a completeness is attained in Chapter III only after the development of a theory of multiplicative systems and lattices of regular amalgamation points. Theorems 14.10 and 14.11 afford a basic technique for this lattice theory. The examples at the end of this section give indications of the extent to which our results may find applications in irregular spaces.

D 14.1. An $S$-portion, $P$ which satisfies the following condition is called an amalgamation point: If $E \in P$ and $X$ is a point element which is a limit element $\left({ }^{15}\right)$ of $E$, then $X$ and $E$ intersect. In the first chapter we defined order in terms of intersection. Since both order and continuity relations are used in our topological applications of the amalgamation points, it may be noted that the definition involves both order and continuity.

D 14.2. If $M$ is a collection of points, $P$ is an amalgamation point, $P<M$, and $M<P$, we say that $P$ is the amalgamation of the elements of $M$. That is, the summation of D 9.3 is called an amalgamation if the result of the summation process is an amalgamation point.

ExAmples. E 14.1. Let $S$ be the plane, $E$ be the set of all decomposition point elements, and $F$ be the set of all boundary elements. By Theorems 13.7, 13.1 and 5.2, $E$ does not intersect $F$. Clearly, each element of $F$ is a limit element of $E$. Thus, $P(E)$ is not an amalgamation point. If $X \in E$, there exists an $S$-neighborhood of $X, R$, whose closure is perfectly compact in itself; by Theorems 10.3 and $11.3 S-\bar{R}$ is an $S$-neighborhood of each boundary element. Thus, $X$ is not a limit element of $F$, and $P(F)$ is an amalgamation point.

$\mathrm{E}$ 14.2. The point $Q(F)$ of $\mathrm{E} 9.2$ is an amalgamation point; it is the amalgamation of all ends of $F$.

The point of $E 8.3$ is an amalgamation point but is not a composition point. The point $P(E)$ of $E 8.2$ is not an amalgamation point; here $F$ is a limit element of $E$ but does not intersect $E$.

Theorem 14.1. An $S$-portion $P$ is an amalgamation point if and only if the following condition holds: If the composition point $X$ is a limit point of the collection of composition points $M$ and $P<M<P$, then $X$ intersects $M$ and $P$.

It may be proved with the help of D 14.1 and Theorems 11.2, 8.1, and 9.1.

Theorem 14.2. A composition point is an amalgamation point.

Proof. Let $E$ and $F$ be non-intersecting point elements; by $\mathrm{D} 4.4$ there

(15) Cf. D 12.1, and D 7.1. 
exist $e \in E$ and $f \in F$ such that $e \cdot f$ is vacuous. Then $R=S-\bar{e} \supset f$. If the point element $G$ belongs to an element of $P(E)$, it intersects itself and $E$ (cf. D 8.1 and Theorem 7.1). If $g \in G, e \cdot g$ is nonvacuous, $R$ does not contain $g$, and $R$ is not an $S$-neighborhood of $G$. Thus, $F$ is not a limit element of any element of $P(E)$. By $\mathrm{D} 14.1 P(E)$ is an amalgamation point.

D 14.3. If $M$ is a point set, $G$ is a collection of open sets in $S$, and for $m \in M$ there exists $g \in G$ such that $g$ is an $S$-neighborhood of $m$, then $G$ is said to cover $M$ (relative to $S$ ).

THEOREM 14.3. Let $M$ be an amalgamable collection of ideal points and let $G$ cover $M$ relative to $S$. Then there exists a finite subcollection of $G$ such that the sum of its elements is an $S$-neighborhood of $M$.

Note that the conclusion does not state that the finite subcollection covers $M$ relative to $S$. The latter is the type of conclusion one has where the BorelLebesgue property holds. Theorem 14.3 is used in establishing the BorelLebesgue property for certain decompositions of an amalgamation point (cf. Theorems 14.4, 14.5, and 15.3).

Proof. Suppose that the conclusion does not hold for $G$. Let $N$ be the aggregate of all atomic ideal points which are portions of elements of $M$. If $R$ is an open set of $S$, let $N(R)$ denote the aggregate of all elements of $N$ of which $R$ is an $S$-neighborhood. Let $H$ be the collection of all $R$ 's such that $N(R)$ is covered relative to $S$ by a finite subcollection of $G$, say $G(R)$. Let the symbol $L$ denote the complement in $S$ of an element of $H$; let $E$ be the aggregate of all $L$ 's, and let $K$ be the product of all the $L$ 's.

It follows from Theorems 8.1 and 9.1 that if an ideal point intersects an element of either $M$ or $N$, then it intersects an element of the other; by D 9.2, $M<N$ and $N<M$; by Theorem 11.6 each $S$-neighborhood of $M$ is an $S$-neighborhood of $N$, and conversely. Since each element of $N$ is a portion of an element of $M$, it follows by Theorem 11.6 that $G$ covers $N$; if $R \in G$, then $R$ covers $N(R)$ relative to $S$; thus $R \in H$, and $H \supset G$. Suppose that $D_{1}$ is the sum of the elements of a finite subcollection of $H$, say $H_{1}$, and $D_{1}$ is an $S$-neighborhood of $M$; then $D_{1}$ is an $S$-neighborhood of $N$; by Theorem 11.7 $H_{1}$ covers $N$ relative to $S$. If $R_{i} \in H_{1}, G\left(R_{i}\right)$ covers $N\left(R_{i}\right)$ relative to $S$. Since $N$ is the sum of the finite collection of $N\left(R_{i}\right)$ 's, $N$ is covered by the sum of the sets $G\left(R_{i}\right)$; this sum, $G^{*}$, is a finite subcollection of $G$. If $D^{*}$ is the sum of the elements of $G^{*}, D^{*}$ is an $S$-neighborhood of $N$ and of $M$. This is contrary to our supposition concerning $G$. Thus, $D_{1}$ and $H_{1}$ do not exist. Thus, the hypothesis of our theorem holds for each of $G$ and $H$, but the conclusion holds for neither.

Suppose that an element of $E, L$, were vacuous; then $S-L=S$ would be an element of $H$; then $N=N(S)$, and $N$ is covered by a finite subcollection of $G, G(S)$; since $H \supset G$ we are involved in a contradiction (cf. the preceding paragraph). Thus, $L$ does not exist. If $R_{1}$ and $R_{2}$ are elements 
of $H$, it follows from Theorem 11.7 that $N\left(R_{1}+R_{2}\right)=N\left(R_{1}\right)+N\left(R_{2}\right)$. Since $S-\left(R_{1}+R_{2}\right)=\left(S-R_{1}\right) \cdot\left(S-R_{2}\right)$, it follows that the product of two elements of $E$ is an element of $E$.

Let $P \in K$. Suppose that $L_{1} \in E$ and $P$ is not a limit point of $L_{1}$. There exists an open set in $S, D$, which contains $P$ but contains no element of $L_{1}-P$. Then $S-L_{1} \supset D-P$ and $N\left(S-L_{1}\right) \supset N(D-P)$. Thus, $D-P \in H$ and $N(D-P)$ is covered relative to $S$ by a finite subcollection of $G$. Since $P \in K \cdot D, S-D$ is not an element of $E, D$ is not an element of $H, N(D)$ is not covered by a finite subcollection of $G$, and $N(D) \neq N(D-P)$. By Theorem 11.4 if $P$ were nonisolated, we should have $N(D)=N(D-P)$; thus, $P$ is isolated. By Theorem 8.4 there exists one atomic ideal point, $\beta$, such that $P=a(\beta)$, and $\beta$ is degenerate (cf. D 12.2 and Theorem 13.7). Thus, $N(D)$ is either $N(D-P$ ) or $\beta+N(D-P)$; in either case it can be covered by a finite subcollection of $G$, and $D \in H$. Therefore, we are involved in a contradiction, and $L_{1}$ does not exist.

Let $P \in K$. For $R$ an $S$-neighborhood of $P$ and $L \in E$ let $F(R, L)$ $=(L-P) \cdot \bar{R}_{S}$, and let $F$ be the aggregate of all $F(R, L)$ 's. Since $P$ is a limit point of each $L$, the product of two $R$ 's is an $R$, and the product of two $L$ 's is an $L$, it follows that the elements of $F$ satisfy conditions (2) and (1) of D 4.1 ; if they also satisfy (4) they satisfy (3), and $F$ is a decomposition point element. Suppose that (4) is not satisfied, and that $U$ is a definite $S$-neighborhood of $P$ which contains no element of $F$. Let $T$ be the aggregate of all sets, $(\bar{U}-U) \cdot F(R, L)$, where $F(R, L) \in F$, and $U \supset R$. By the definition of $U$ no element of $T$ is vacuous. Since the product of two elements of $F$ is an element of $F$, the product of two elements of $T$ is an element of $T$. Let $Y \in \bar{U}-U$; since $S$ is a Hausdorff space, there exist in it mutually exclusive open sets, $V$ and $W$, which contain $Y$ and $P$. If $R=U \cdot W$ and $L \in E$, then $(\bar{U}-U) \cdot F(R, L)$ does not contain $Y$; thus, the product of the elements of $T$ is vacuous; by $\mathrm{D} 4.1$ $T$ is a boundary element. Similarly, if $K$ is vacuous, $E$ is a boundary element. Let $\Sigma$ denote any of the three $F, T$, or $E$ which is a point element; we have shown that at least one of them satisfies this condition. $\Sigma$ has the property that if $L \in E$ then $L$ contains an element of $\Sigma$. Let $\beta$ be the composition point of which $\Sigma$ is an element.

Let $X \in N$ and let $Y$ be a point element which is an element of $X$ (cf. Theorem 8.2 and D 8.4). There exists $g_{X} \in G$ such that $g_{X}$ is an $S$-neighborhood of $X$ (for, $G$ covers $N$ relative to $S$ ). Since $H \supset G, S-g_{X} \in E, S-g_{X}$ contains an element of $\Sigma$, and $g_{X}$ contains an element of $Y$; by D $4.4 \Sigma$ does not intersect $Y$; by Theorem $8.1 \beta$ does not intersect $X$. Since $N<M<N$ and $M$ is amalgamable so is $N$. By Theorems 9.1 and $14.1 \beta$ is not a limit point of $N$. By Theorem 12.2 there exists an $S$-neighborhood of $\beta, D$, which is an $S$-neighborhood of no element of $N$. There exists $\Sigma_{1} \in \Sigma$ such that $D \supset \Sigma_{1}$. Since $N(D)$ is vacuous, $D \in H, S-D \in E$, and $S-D \supset \Sigma_{2}$, where $\Sigma_{2} \in \Sigma$. Since $\Sigma_{1} \cdot \Sigma_{2}$ is vacuous, we are involved in a contradiction. 
TheOREM 14.4. An amalgamable collection of atomic ideal points has the Borel-Lebesgue covering property and is perfectly compact in itself.

By Theorems 14.3 and 11.7 the set has the covering property. It is perfectly compact in itself; cf. Theorem 12.1 and Kuratowski and Sierpiński, loc. cit., Bibliography.

THEOREM 14.5. The aggregate of all atomic ideal points is amalgamable and is perfectly compact in itself. The amalgamation of this collection is regular, and it is the maximal ideal point.

Proof. Let $M$ be the collection. By Theorem 9.1 the summation of the elements of $M, \beta$ exists. If $P$ and $Q$ are intersecting ideal points, by Theorem $9.2 \alpha(P) \cdot \alpha(Q)$ is nonvacuous; then $M \supset \alpha(P) \cdot \alpha(Q)$; by Theorems 9.2 and 9.1 $Q$ intersects $\beta$ and $M$; by $\mathrm{D} 9.2 P<M$. By Theorem $14.1 \beta$ is an amalgamation point. Clearly, $a(\beta)$ consists of all points of $S$ (cf. D 12.2 and D 9.4). By D 13.1 $\beta$ is regular.

THeORem 14.6. Let $P$ be a real point, $Q$ be an amalgamation point, and $M$ be a collection of composition points such that $Q<M<Q$. The following conditions are equivalent: (1) $P$ and $Q$ do not intersect; (2) no element of $M$ intersects $P$; (3) $P$ and $Q$ can be separated by $S$-neighborhoods.

Proof. Let $X \in M$ and $E$ be a point element which belongs to some element of $X$. Since $X<Q$, by Theorem $8.1 E$ belongs to an element of $Q$. By D 9.1 if $X$ intersects $P$, then so does $Q$; thus condition (1) implies condition (2). By condition (2) and Theorem $11.5 X$ and $P$ can be separated by a pair of their respective $S$-neighborhoods, $U(X)$ and $V(X)$. By Theorem 14.3 there exists a finite collection of the $U(X)$ 's such that the sum of these, $U$, is an $S$-neighborhood of $M$. If $V$ is the product of the corresponding $V(X)$ 's, $V$ is an $S$-neighborhood of $P$, and $U \cdot V$ is vacuous. By Theorem $11.6 U$ is an $S$ neighborhood of $Q$. Thus, (2) implies (3). By D 11.1, D 11.2, D 9.1 and D 4.5, (3) implies (1).

THEOREM 14.7. If $P$ and $Q$ are amalgamation points, one of which is regular, the following are equivalent: (1) $P$ does not intersect $Q$; (2) $P$ intersects no element of $\alpha(Q)$; (3) $P$ and $Q$ are separated by $S$-neighborhoods.

Proof. By D 11.1, D 11.2, D 7.1, and D 4.4, and Theorem 8.1, (3) implies (1). By Theorem 9.1, (1) and (2) are equivalent; to establish (3) we shall consider the case of $Q$ being regular. Let $X \in \alpha(P), Y \in \alpha(Q)$, and $E$ and $F$ be point elements such that $E \in X$ and $F \in Y$ (cf. Theorem 8.2). By Theorem 8.1 $E$ does not intersect $F$; there exist $e(Y) \in E$ and $f \in F$ such that $f \cdot e(Y)$ is vacuous. Then by $\mathrm{D} 4.1 D(Y)=S-\overline{e(Y)} S \supset f$, and $D(Y)$ is an $S$-neighborhood of $F$ and of $Y$. By Theorems 9.1 and 14.3 there exist $Y_{1}, Y_{2}, \cdots, Y_{n}$ such that $D=D\left(Y_{1}\right)+D\left(Y_{2}\right)+\cdots+D\left(Y_{n}\right)$ is an $S$-neighborhood of $\alpha(Q)$ and of $Q$ (cf. Theorem 11.6). By D 13.1 there exists an $S$-neighborhood of $Q, R$, such that 
$D+a(Q) \supset \bar{R}_{S}$. By Theorem $9.1 Q<\alpha(Q)<Q$; the methods of the proof of Theorem 12.4 show that $a(Q)=a(\alpha(Q))$. If each element of $E$ contained points of $a(Q)$, by D 4.1, D 11.1, and D $12.2 X$ would be a limit point of $\alpha(Q)$; by Theorem 14.1 $X$ intersects $\alpha(Q)$ and $Q$. By (1) this is impossible, and there exists $e_{0} \in E$ such that $a(Q) \cdot e_{0}$ is vacuous. By D 4.1 there exists $e \in E$ such that $e_{0} \cdot e\left(Y_{1}\right) \cdot e\left(Y_{2}\right) \cdots e\left(Y_{n}\right) \supset e$. Then $e \cdot(D+a(Q))$ is vacuous; $S-\bar{R}_{S}$ contains $e$ and is an $S$-neighborhood of $E$ and $X ; X$ and $Q$ can be separated by $S$-neighborhoods. The conclusion for (3) follows by methods used for Theorem 14.6.

THEOREM 14.8. A regular amalgamation point which is a portion of either a decomposition point or of a collection of boundary points is a composition point.

Proof. Let $P$ be the amalgamation point and $Q$ be a decomposition point such that $P<Q$. Let $E \in P$; let $F$ and $G$ be point elements such that $F \in Q$ and $G$ belongs to $E$; let $F(P)$ and $Q(P)$ be defined as in Theorem 13.7. By Theorem 7.2 and D $8.2 G<E<F$. By D $7.1 G$ intersects $F$. By Theorems 5.1 and 12.4, and D 12.2, $a(Q)=a(F)=a(G)=a(E)=a(P)$. If $R$ is an $S$-neighborhood of $a(P)$, it is an $S$-neighborhood of $F, Q$, and $P$ (cf. D 4.1, D 11.1, and Theorem 11.6). Since $P$ is regular, it has an $S$-neighborhood $U$ such that $R+a(P) \supset \bar{U}_{S}$. Then $\bar{U}_{S}$ or $\bar{U}_{S}-a(P)$ is an element of $F(P)$. By D $4.1 F(P)$ is a point element, and $a(P)=a(F(P))$.

Consider the second hypothesis for $P$. Let $X \in S, R=S-X$; by an argument similar to that of the preceding paragraph, it follows that $a(P)$ is vacuous, and $F(P)$ is a boundary element.

Since each element of $F(P)$ contains an $S$-neighborhood of $P$, each element of $F(P)$ contains elements of each point element which belongs to $E$. Thus, $E<F(P)$, and $P<Q(P)$; cf. Theorem 7.1 and D 8.2. Suppose that not $Q(P)<P$; by Theorem 9.2 there exists $Y \in \alpha(Q(P))-\alpha(P)$, and $Y$ does not intersect $P$. By Theorem $14.7 P$ and $Y$ can be separated by $S$-neighborhoods $U$ and $V$. Since $\bar{U}$ or $\bar{U}-a(P)$ is an element of $F(P)$, and $F(P) \in Q(P), F(P)$ intersects no point element of which $V$ is an $S$-neighborhood. By Theorem 8.1 $Y$ and $Q(P)$ do not intersect. Thus, the supposition that "not $Q(P)<P$ " involves a contradiction. Since, also, $P<Q(P), P=Q(P)$.

TheOREM 14.9. If $D$ is an $S$-neighborhood of the regular amalgamation point $P$, then the set $(S-D) \cdot a(P)$ is finite or vacuous.

Proof. Suppose that $M=(S-D) \cdot a(P)$ is infinite. By Theorems 10.3, 10.4, and 12.3 there exists a composition point $\beta$ which is an end and a limit point of $M$; since $S-D \supset M, \beta$ is an end of $S-D$ (cf. D 10.2). By Theorems 10.2 and 11.3 $P$ and $\beta$ do not intersect. By Theorem 14.7P and $\beta$ can be separated by $S$-neighborhoods $U$ and $V$. Since $\beta$ is a limit point of $M, V$ contains points of $M$ and of $a(P)$; by Theorem 12.4 $\bar{U} \supset a(P)$. Thus, the supposition that $M$ is infinite involves a contradiction. 
THEOREM 14.10. (1) A perfectly compact set of regular amalgamation points is amalgamable. (2) An amalgamation of a collection of regular amalgamation points is regular.

Proof. By Theorem 9.1, if $N$ is the set of (1), then $N$ has a summation $P$; $N<P<\alpha(P)<N$. If the composition point $X$ intersects no element of $\alpha(P)$, by D $9.2 X$ intersects no element of $N$. By Theorem 14.7 each element of $N$ can be separated from $X$ by $S$-neighborhoods. There exists a finite collection $H$ of open sets which covers $N$ relative to $S$, and an $S$-neighborhood of $X, U$, such that $U$ has no point in common with an element of $H$ (cf. the proof of Theorem IX, p. 89, Alexandroff and Hopf). Let $W$ be the sum of the elements of $H$; it is an $S$-neighborhood of $N$ and of $\alpha(P)$ (cf. Theorem 11.6). Thus, $X$ is not a limit point of $\alpha(P)$. By Theorem 14.1 $P$ is an amalgamation point.

Let the elements of $N$ be regular, and let $D$ be an $S$-neighborhood of $P$. If $X \in N, D$ is an $S$-neighborhood of $X$ and $a(P) \supset a(X)$ (cf. Theorems 11.6 and 12.4). By our hypothesis there exists an $S$-neighborhood of $X, U(X)$, such that $D+a(X) \supset \overline{U(X)}$. By Theorems 14.3 and 11.6 there exists a finite collection of the $U(X)$ 's, whose sum $U$ is an $S$-neighborhood of $N$ and of $P$. Then $D+a(P) \supset \bar{U}$.

THEOREM 14.11. (1) If a collection $H$ of amalgamation points has a lower bound, its greatest lower bound is an amalgamation point; (2) if the elements of $H$ are regular and one of them is the amalgamation of a set of regular composition points, then their greatest lower bound is regular.

Theorems $14.5,14.10$, and 14.11 are applied in Chapter III in questions concerned with the existence and the properties of multiplicative systems and complete lattices of regular amalgamation points. Examples may be given to show that the conclusion of (2) does not hold if the second part of the hypothesis be omitted.

Proof. Let $F$ be the product of all the $\alpha(h)$ 's, where $h \in H$. If $P$ is the summation of the elements of $F$, by Theorem $9.2 P$ is the greatest lower bound of the $h$ 's. Let the composition point $Q$ be a limit point of $F$ which does not intersect $P$. Suppose that no element of $\alpha(Q)$ is a limit point of $F$. By Theorem 12.2 , if $\beta \in \alpha(Q)$, there exists an $S$-neighborhood of $\beta, D(\beta)$, which is not an $S$-neighborhood of any element of $F$. By Theorems 14.2, 14.3, and 11.6 there exists an $S$-neighborhood of $Q, D$, which is the sum of a finite collection of the $D(\beta)$ 's. By Theorem 11.7 $D$ is not an $S$-neighborhood of any element of $F$. Thus, we are involved in a contradiction, and there exists $\beta \in \alpha(Q)$ such that $\beta$ is a limit point of $F$. If $h \in H, \alpha(h) \supset F$, and $\beta$ is a limit point of $\alpha(h)$; by Theorem $14.1 \beta$ intersects an element of $\alpha(h)$. Since $\beta$ is atomic, $\beta \in \alpha(h)$. Thus, $\beta \in F$; by Theorem 8.1 $P$ and $Q$ intersect. By Theorem 14.1 $P$ is an amalgamation point. 
We shall first establish (2) for the case where the number of elements of $H$ is the positive integer $n$. Clearly (2) holds for $n=1$. Suppose that (2) holds for each collection with fewer than $n$ elements. Let $A$ be an element of $H$ which is the amalgamation of a collection $T$ of regular amalgamation points; Let $B \in H-A$, and $H^{\prime}=H-B$. By the definition of $n$, the elements of $H^{\prime}$ have a greatest lower bound $C$, which is a regular amalgamation point. Let $Z$ be the greatest lower bound of $B$ and $C$. By Theorem $9.2 Z$ is the greatest lower bound of the elements of $H$; by part (1) $Z$ is an amalgamation point. Let $D$ be an $S$-neighborhood of $Z$. If $X$ is an amalgamation point, let $D(X)$ denote the aggregate of all elements of $\alpha(X)$ of which $D$ is not an $S$-neighborhood. By Theorem $14.4 \alpha(X)$ is perfectly compact in itself. Since $D(X)$ is closed relative to $\alpha(X)$, it is perfectly compact in itself (cf. Fréchet, (I), pp. 229230). By Theorem $11.6 D$ is an $S$-neighborhood of $\alpha(Z)$; by Theorem 9.2 $\alpha(Z)=\alpha(B) \cdot \alpha(C)$; it follows that $D(B) \cdot D(C)$ is vacuous. If $X \in D(B)$, by Theorem $8.1 X$ does not intersect $C$. By the definition of $n, C$ is regular; by Theorem $14.7 X$ and $C$ can be separated by $S$-neighborhoods. By Theorem 11.6 each element of $D(B)$ can be separated from each element of $D(C)$ by $S$-neighborhoods. Since each of $D(B)$ and $D(C)$ is perfectly compact in itself, they can be separated by $S$-neighborhoods $D_{1}$ and $D_{2}$ (apply an argument similar to that for the proof of Theorem IX, p. 89, Alexandroff and Hopf). Then $D+D_{1}$ and $D+D_{2}$ are $S$-neighborhoods, respectively, of $\alpha(B)$ and of $B$, and of $\alpha(C)$ and of $C$, respectively. Since $B$ and $C$ are regular, there exist $S$-neighborhoods $R_{1}$ and $R_{2}$ of $B$ and $C$, respectively, such that $E_{1}=D+D_{1}$ $+a(B) \supset \bar{R}_{1}$, and $E_{2}=D+D_{2}+a(C) \supset \bar{R}_{2}$. Since $Z<B$ and $Z<C$, by Theorem $12.1 R=R_{1} \cdot R_{2}$ is an $S$-neighborhood of $Z$. Let $M_{1}=D+D_{1}$, and $N_{1}=\left(S-M_{1}\right) \cdot a(B)$; thus, $M_{1}+N_{1}=E_{1}$. Let $M_{2}=D+D_{2}, N_{2}=\left(S-M_{2}\right) \cdot a(C)$, and $K=M_{1} \cdot N_{2}+M_{2} \cdot N_{1}+N_{1} \cdot N_{2}$. By Theorem $14.9 N_{1}, N_{2}$, and $K$ are finite or vacuous. Since $D_{1} \cdot D_{2}$ is vacuous, $M_{1} \cdot M_{2}=D$, and $E_{1} \cdot E_{2}=\left(M_{1}+N_{1}\right)$ $\cdot\left(M_{2}+N_{2}\right)=D+K$. Thus, $D+K \supset \bar{R}$. By Theorem $12.4 \quad \bar{R} \supset a(Z)$ and $a(B) \cdot a(C) \supset a(Z)$. Let $L=K-K \cdot a(Z)$; then $L$ is finite or vacuous. Suppose that there exists $X \in \alpha(Z)$ and $Y \in L$ such that $X$ intersects $Y$. Since $X<Z<A, X \in \alpha(A)$. Since $A<T$, by D $9.2 X$ intersects an element of $T$, say $X_{1}$. Let the point elements $E$ and $E_{1}$ be elements of $X$ and of $X_{1}$, respectively. By Theorem $8.1 E$ and $E_{1}$ intersect; by Theorem $5.1 a(E)=a\left(E_{1}\right)$; by D $12.2 a(X)=a\left(X_{1}\right)$. Since $X<Z$, by Theorem $12.4 a(Z) \supset a(X)$; thus, $a\left(X_{1}\right) \neq Y$. By $\mathrm{D} 4.1$ there exists $e_{1} \in E_{1}$ such that $e_{1}$ does not contain $Y$. Then $S-Y$ is an $S$-neighborhood of $E_{1}$ and of $X_{1}$. Since $X_{1}$ is regular, there exists an $S$-neighborhood of $X_{1}, U$, such that $S-Y \supset \bar{U}$; then $S-\bar{U}$ is an $S$-neighborhood of $Y$. Since $X$ is atomic and intersects $X_{1}, X<X_{1}$; then $U$ is an $S$-neighborhood of $X$. By Theorem $11.5 X$ does not intersect $Y$; thus the supposition that $X$ and $Y$ exist involves a contradiction. By Theorem 14.6 $Z$ and $L$ can be separated by a pair of their respective $S$-neighborhoods, $U$ and $V$. Then $W=R \cdot U$ is an $S$-neighborhood of $Z, \bar{R} \supset \bar{W}$, and $\bar{W} \cdot L$ is vacu- 
ous; since $D+K \supset \bar{R}, D+a(Z) \supset D+(K-L) \supset \bar{W}$. Thus, $Z$ is regular. Thus, by induction we establish the conclusion of (2) for any case where $H$ is finite.

Consider the infinite case. Let $Z$ be the lower bound of the elements of $H$; let $A, D$, and $D(X)$ be defined as in the preceding paragraph. By Theorem 9.2 $\alpha(Z)$ is the product of the sets $\alpha(h)$, where $h \in H$; by Theorems 9.1 and 11.6 $D$ is an $S$-neighborhood of $\alpha(Z)$; hence, the sets $D(h)$ have a vacuous product. We showed in the preceding paragraph that each of the $D(h)$ 's is perfectly compact in itself; it follows that there exists a finite subcollection of $H$, $H^{\prime}=\left(h_{1}, h_{2}, \cdots, h_{n}\right)$, such that the product $\lambda=D\left(h_{1}\right) \cdot D\left(h_{2}\right) \cdots D\left(h_{n}\right)$ is vacuous (cf. Fréchet, (I), p. 231). We shall suppose that $A \in H^{\prime}$; this is permissible, for if the product $\lambda$ is vacuous, the product of all the elements of $\lambda$ by $D(A)$ will also be vacuous. Let $J$ be the product $\alpha\left(h_{1}\right) \cdot \alpha\left(h_{2}\right) \cdot \alpha\left(h_{3}\right) \cdots \alpha\left(h_{n}\right)$. Let $B=C=P(J)$; by the results of the preceding paragraph $B$ is the greatest lower bound of the elements of $H^{\prime}$, and it is a regular amalgamation point. Clearly $D$ is an $S$-neighborhood of each element of $J$ and of $J$; by Theorem 11.6 $D$ is an $S$-neighborhood of $B$. Let $R$ be an $S$-neighborhood of $B$ such that $D+a(B) \supset \bar{R}$; let $K=(S-D) \cdot a(B)$ and $L=K-K \cdot a(Z)$. Then $D+K \supset \bar{R}$. By following the argument of the later portion of the preceding paragraph, we see that $Z$ is a regular amalgamation point.

THEOREM 14.12. (1) A Hausdorff space is normal if and only if all its atomic boundary points are regular; (2) it is semi-completely normal if and only if all its atomic ideal points are regular.

Proof. By Theorems 13.2 and 13.3 the conditions are necessary. By Theorem 14.2 each composition point is an amalgamation point. If the conditions hold it follows from Theorems $14.10,14.2$, and 9.1 that each of the composition points involved in the conditions of Theorems 13.2 and 13.3 is regular. Thus, the conditions are sufficient.

THEOREM 14.13. All the amalgamation points of a semi-completely normal space are regular, and each of them can be decomposed into a collection of regular atomic composition points.

This follows from Theorems 9.1, 14.12 and 14.10.

ExAmpLEs. E 14.3. Urysohn $\left({ }^{16}\right)$ has constructed a countable space $R$ such that if $X$ and $Y$ are points of $R$ and $U$ and $V$ are open sets containing $X$ and $Y$, respectively, then $\bar{U} \cdot \bar{V}$ is nonvacuous; because of this condition no point of $R$ is isolated. Let $P$ be an amalgamation point which is distinct from the maximal amalgamation point of $R$; cf. D 9.4 and Theorem 14.5. Suppose that $P$ is regular. By D 9.2 and Theorem 8.1 there exists a composition point $Q$ which does not intersect $P$. By Theorems 14.2 and $14.7 P$ and $Q$ can be separated by a pair of their respective $R$-neighborhoods, $U$ and $V$. There exists an

(16) Loc. cit., pp. 274-283. 
$R$-neighborhood of $P, W$, such that $U+a(P) \supset \bar{W}$. By Theorem 14.9 there exist at most a finite number of points in $(R-U) \cdot a(P)$, say $P_{1}, P_{2}, \cdots, P_{n}$. Since no point of $R$ is isolated it follows from Theorems 12.3,11.3, and 11.6 that each of $P$ and $Q$ is a limit point of $R$. Thus, there exist $X \in V$ and $Y \in W$. For each $i$ let $\delta_{i}$ and $\lambda_{i}$ be mutually exclusive open sets which contain $P_{i}$ and $X$ respectively. Let $\lambda=V \cdot \lambda_{1} \cdot \lambda_{2} \cdots \lambda_{n}$. Then $\bar{V} \supset \bar{\lambda}, U \cdot \bar{V}$ is vacuous, $a(P) \cdot \bar{\lambda}$ and $\bar{W} \cdot \bar{\lambda}$ are vacuous; we are involved in a contradicion. To summarize: the maximal ideal point is a regular amalgamation point of $R$ and is the only one; by Theorem 14.2 no composition point of $R$ is regular.

15. The upper semi-continuity and the perfect compactness of decompositions. First we shall recall definitions by Moore and by Alexandroff( $\left.{ }^{17}\right)$, and then we shall introduce extensions of these. (I) Let $T$ be a space $H$ Fréchet, $K$ be a point set of $T$, and $M$ be a collection of mutually exclusive subsets of $K$ whose sum is $K ; M$ is called a decomposition (Zerlegung) of $K$ (cf. D 9.3). (II) A collection $M$ of mutually exclusive closed point sets of $T$ is said to be upper semi-continuous (in $T$ ) provided that if $P \in M$ and $D$ is an open set of $T$ which contains $P$, there exists in $T$ an open set $R$ which contains $P$ such that if an element of $M$ has a point in common with $R$ then that element is a subset of $D\left({ }^{18}\right)$. (III) If $T \supset K$ and $M$ is a decomposition of $K$, then $T(M)$, the (weak) space of the decomposition, is defined as follows: (1) its points are the elements of $M$; (2) if $U$ is an open point set in $T$, let $U(M)$ be the aggregate of all elements of $M$ which are subsets of $U$; the $U(M)$ 's are the neighborhoods for $T(M){ }^{(19)}$ (cf. Theorem 12.1). (IV) A continuous mapping of the space $X$ on the space $Y$ is said to be closed if the image of each closed point set in $X$ is a closed point set in $Y\left({ }^{20}\right)$.

EXAmples. E 15.1. Let $X$ be the subspace of the plane whose points are those of a circle and its interior. Let $\delta$ be a definite diameter of the circle; Let $Y_{1}$ be the decomposition of $X$ which consists of $\delta$ and all chords of the circle which are parallel to $\delta$; let $Z_{1}$ be the decomposition whose elements are the points of $\delta$, and the chords that are parallel to $\delta$. Let $Y$ and $Z$ be the spaces of the decompositions $Y_{1}$ and $Z_{1}$ respectively. Let the relations $y=f(x)$ and $z=g(x)$ mean, respectively, that $x \in y$ and $x \in z$. The collection $Y_{1}$ is upper semi-continuous in $X$, but $Z_{1}$ is not. The spaces $X$ and $Y$ are perfectly compact, but $Z$ is not. The mapping $y=f(x)$ of $X$ on $Y$ is closed. The mapping

${ }^{17}$ ) Cf. Moore, (III), and Alexandroff, (I). For treatments of the theory see Moore, (I), chap. 5, and Alexandroff and Hopf, pp. 61-70 and 95-98.

(18) Moore, loc. cit.; Alexandroff and Hopf call $M$ a continuous decomposition (stetige Zerlegung) of the sum of the elements of $M$ (loc. cit., p. 67).

(19) Alexandroff and Hopf, p. 66, call $T(M)$ der schwache Zerlegungsraum der Zerlegung $M$. If $M$ is upper semi-continuous, $T(M)$ is their Zerlegungsraum (loc. cit., pp. 63 and 67). We deal mainly with the latter.

${ }^{(20)}$ Alexandroff and Hopf, p. 95; for information and definitions having to do with mappings (Abbildungen) see pages 51,52 ; in $\mathrm{V}$, page 54 , they show that a continuous mapping need not be closed. 
$z=g(x)$ of $X$ on $Z$ is not continuous. The mapping $y=f\left(g^{-1}(z)\right)$ of $Z$ on $Y$ is continuous but is not closed. The example both serves to illustrate the results of Theorem 15.1 and to give Gegenbeispiele. If we amalgamate the elements of $Y_{1}$ and $Z_{1}$, we obtain collections of amalgamation points which may be used to illustrate Theorem 15.4.

The extensive results of Alexandroff and Hopf in their Theorem VIII, p. 98, may be amplified by the following theorem. The mapping of their hypothesis is continuous rather than closed. They give no result analogous to that of the Conditions (I) and (III) implying (II); the transformation $y=f\left(g^{-1}(z)\right)$ of the preceding example illustrates the fact that the stronger condition, closedness, is essential for such a result.

Theorem 15.1. Let $X$ and $Y$ be Hausdorff spaces, and let $y=\alpha(x)$ define $a$ continuous mapping of $X$ into $Y$; then any two of the following conditions imply the third: (I) $Y$ is perfectly compact; (II) $X$ is perfectly compact; (III) the mapping $y=\alpha(x)$ is closed; for $y \in Y$ the set $\alpha^{-1}(y)$ is perfectly compact in itself.

Proof. By Alexandroff and Hopf, Theorem VIII, p. 98, Conditions (II) and (III) imply (I). By this theorem and their Theorems II on page 95, (I) on page 53, and IV on page 86 Conditions (I) and (II) imply (III). Let $H$ be a collection of open sets which covers $X$. If $y \in Y$ let $H(y)$ be a finite subcollection of $H$ which covers $\alpha^{-1}(y)$ (cf. Condition (III) and Kuratowski and Sierpiński, loc. cit.). Let $D(y)$ be the sum of the elements of $H(y)$; then $X-D(Y)$ is closed in $X$. By (III) $\alpha(X-D(y))$ is closed in $Y$; and $\delta(y)=Y-\alpha(X-D(y))$ is open in $Y$ and contains $y$. By (I) there exists a finite subcollection of the $\delta(y)$ 's which covers $Y$, say $\delta\left(y_{1}\right), \delta\left(y_{2}\right), \cdots, \delta\left(y_{n}\right)$. Let $H^{*}=H\left(y_{1}\right)+H\left(y_{2}\right)+\cdots+H\left(y_{n}\right)$. If $x_{0} \in X$, there exists $j$ such that $\alpha\left(x_{0}\right) \in \delta\left(y_{j}\right)$; if $x_{0}$ were not covered by $D\left(y_{j}\right)$, it would belong to $X-D\left(y_{j}\right)$; then $\alpha\left(x_{0}\right) \in \alpha\left(X-D\left(y_{j}\right)\right)=Y-\delta\left(y_{j}\right)$, and we are involved in a contradiction. Thus $x_{0}$ is covered by an element of $H\left(y_{j}\right)$, and $X$ is covered by $H^{*}$. Thus, (I) and (III) imply (II).

D 15.1. A collection $M$ of points is said to be upper semi-continuous (relative to $S$ ) provided that if $P \in M$ and $D$ is an $S$-neighborhood of $P$, there exists an $S$-neighborhood of $P, R$, such that if $Q$ is an element of $M$ and $R$ is an $S$-neighborhood of a portion of $Q$, then $D$ is an $S$-neighborhood of $Q$ (cf. Definition (II) above).

Examples. E 15.2. Let $M$ be a decomposition of the perfectly compact Hausdorff space $S$ into closed sets. Let $N$ be the collection of those ideal points which are amalgamations of elements of $M$. Then $N$ is upper semi-continuous relative to $S$ if and only if $M$ is upper semi-continuous in $S$. Examples of both possibilities are given by Alexandroff and Hopf, p. 67. See also E 15.1.

$\mathrm{E}$ 15.3. A collection of real points, or of atomic ideal points, is upper semicontinuous relative to $S$.

If, in Theorem 15.1, we amalgamate the elements of the sets $\alpha^{-1}(y)$, for 
$y \in Y$, we obtain amalgamation points for which Theorem 15.4 applies. This is an instance of the analogy we have noted before between the properties of perfectly compact sets and of amalgamation points. Similarly, the results of Theorem 15.3 resemble some of those of Alexandroff and Hopf. These analogies give indications of some rather trivial applications of our theory. In general, our procedure is to define mappings in terms of decompositions or of amalgamations; these processes involve mainly order relations. This procedure must be carried out in such a manner that it gives conditions which assure the continuity of the mappings and permit the application of the results of Alexandroff and Hopf. Such conditions are given in Theorems 15.4, 15.5, and 15.6. Thus, we have on the one hand the processes of amalgamation of points and of continuous mappings, and on the other those of decomposition of points and of the inverse of a continuous mapping. We may think of the latter as giving a kind of representation theory $\left({ }^{21}\right)$ for our system of points, in which the representation of a point is a decomposition of that point into an upper semi-continuous collection of amalgamation points. Thus, this representation theory involves not only order, but also continuity.

THEOREM 15.2. If $N$ is an upper semi-continuous collection of amalgamation points, no two of them intersect.

Suppose that $X$ and $Y$ are intersecting elements of $N$. By D $15.1 X$ and $Y$ have the same $S$-neighborhoods. By Theorem 9.2 if not $X<Y$ then there exists an element $\beta$ of $\alpha(X)-\alpha(X) \cdot \alpha(Y)$. By Theorem 12.2 if $\lambda \in \alpha(Y)$ there exists an $S$-neighborhood of $\lambda, \delta(\lambda)$, which is not an $S$-neighborhood of $\beta$. By Theorem 14.3 there exists a finite collection of the $\delta(\lambda)$ 's, whose sum $D$ is an $S$-neighborhood of $Y$. By Theorem $11.7 D$ is not an $S$-neighborhood of $\beta$. Since $\beta<X$ and $D$ is an $S$-neighborhood of $X$, we are involved in a contradiction. Thus, $X<Y$. Similarly, $Y<X$. Hence, $Y=X$.

Theorem 15.3. Let $Y$ be a decomposition(22) $[x]$ of the ideal point $X$ into regular amalgamation points. The following conditions are equivalent: (1) $Y$ is perfectly compact in itself; (2) $X$ is an amalgamation point and $Y$ is upper semicontinuous relative to $S$; (3) the space $\left.{ }^{23}\right) S(Y)$ of the decomposition $Y$ is a perfectly compact Hausdorff space.

The following is a point set analogue of this theorem.

THeOREM 15.3A. If $Y$ is a decomposition of the Hausdorff space $X$ into a collection of sets which are perfectly compact in themselves, the following conditions are equivalent: (1) The space $X(Y)$ of the decomposition $Y$ is perfectly compact; (2) $X$ is perfectly compact and $Y$ is upper semi-continuous in $X$.

(21) Cf. Birkhoff, p. 76, and Stone, loc. cit.

(22) Cf. D 9.3.

${ }^{(23)}$ Cf. Theorem 12.1. 
Consider 15.3A. By Alexandroff and Hopf, p. 98, Theorem VIII, (2) implies (1). Let (1) hold and $y=\alpha(x)$ mean that $x \in y \in Y(X \supset y)$. If $U$ is an open set in $X, U(Y)$ means the set of all elements of $Y$ that are subsets of $U$; and $U(Y)$ is an open set in $X(Y)$ (cf. Definition (III)). Let $y \in Y$, and $R$ be an open set in $X$ containing $y$. If $z \in Y-R(Y)$, there exist in $X$ mutually exclusive open sets, $V_{z}$ and $W_{z}$, which contain, respectively, $y$ and $z$ (cf.proof, Theorem IX, p. 89, Alexandroff and Hopf). Since $Y-R(Y)$ is a closed set in the perfectly compact space $X(Y)$, it has the Borel-Lebesgue property; there exist $W_{z_{1}}(Y), W_{z_{2}}(Y), \cdots, W_{z_{n}}(Y)$ which cover $Y-R(Y)$ in $X(Y)$. Let $V=V_{z_{1}} \cdot V_{z_{2}} \cdots V_{z_{n}}$ and $W=W_{z_{1}}+W_{z_{2}}+\cdots+W_{z_{n}}$. Then $V$ and $W$ are mutually exclusive open sets in $X, V \supset y$, and $W$ contains all elements of $Y-R(Y)$. Since $V \cdot W$ is vacuous, any element of $Y$ that has a point in common with $V$ is a subset of $R$. Thus, $Y$ is upper semi-continuous in $X$; and the mapping $y=\alpha(x)$ of $X$ on $X(Y)$ is continuous (cf. Alexandroff and Hopf, p. 67). Let $F$ be a closed point set in $X$, and $D=X-F$. Then $D(Y)$ is open in $X(Y)$. Since $D(Y)=X(Y)-\alpha(F), \alpha(F)$ is closed in $X(Y)$, and the mapping is closed. By Theorem 15.1 Condition (1) implies (2).

Proof of Theorem 15.3. Let (2) hold and $G$ be a collection of open sets of $S$ that covers $Y$ relative to $S$. If $x \in Y$, let $D \in G$ such that $D$ is an $S$-neighborhood of $x$. Let $R(x, D)$ be an $S$-neighborhood of $x$ such that if it is an $S$-neighborhood of a portion of an element of $Y$, then $D$ is an $S$-neighborhood of that element. By Theorems 14.4, 11.6, and 9.1 there exists a finite collection of the $R$ 's which covers the aggregate of atomic portions of $X$; say $R\left(x_{1}, D_{1}\right)$, $R\left(x_{2}, D_{2}\right), \cdots, R\left(x_{n}, D_{n}\right)$. Let $G^{*}$ be the collection $D_{1}, D_{2}, \cdots, D_{n}$. If $x \in Y$ and $\beta$ is an atomic portion of $x, \beta<x<X$, and there exists $j$ such that $R\left(x_{j}, D_{j}\right)$ is an $S$-neighborhood of $\beta$. Then $D_{j}$ is an $S$-neighborhood of $x$, and $G^{*}$ covers $Y$ relative to $S$. Since $Y$ has the Borel-Lebesgue covering property, it is perfectly compact in itself (cf. Theorem 12.1 and Kuratowski and Sierpiński, loc. cit.). Thus (2) implies (1).

Conversely, let $Y$ be perfectly compact in itself. By Theorem $14.10 \mathrm{X}$ is an amalgamation point. Let $x \in Y, D$ be an $S$-neighborhood of $x$, and $L$ be the set of all elements of $Y$ of which $D$ is not an $S$-neighborhood. By D 12.1 no point of $Y-L$ is a limit point of $L$, and $L$ is a closed point set relative to $Y$. Since $Y$ is perfectly compact in itself, so is $L$. By Theorem 14.10 there exists a regular amalgamation point $\beta$, such that $\beta<L<\beta$. By D $9.3 x$ intersects no element of $L$; and thus it does not intersect $\beta$. By Theorem 14.7 $x$ and $\beta$ can be separated by a pair of their respective $S$-neighborhoods, $U$ and $W$; by Theorem $11.6 W$ is an $S$-neighborhood of $L$. Let $x_{1}$ be an element of $Y$ such that $U$ is an $S$-neighborhood of a portion $\lambda$ of $x_{1}$. If $x_{1}$ were an element of $L, W$ would be an $S$-neighborhood of $x_{1}$ and of $\lambda$; since $U \cdot W$ is vacuous, this involves a contradiction. Since $x_{1} \in Y-L, D$ is an $S$-neighborhood of $x_{1}$. Thus, $Y$ is upper semi-continuous, and (1) implies (2).

If $x_{1}$ and $x_{2}$ are two elements of $Y$, by Theorem 14.7 and D 9.3 they can 
be separated by $S$-neighborhoods. By Theorem 12.1 condition (1) implies (3). The converse is obvious.

TheOREM 15.4. Given that $P$ is an amalgamation point and $Y$ and $X$ are decompositions of $P$ into regular amalgamation points such that if $y \in Y$ there exists a subcollection $\alpha^{-1}(y)$ of $X$ which is a decomposition of $y$; then any two of the following conditions imply the third: (1) $Y$ is perfectly compact in itself; (2) $X$ is perfectly compact in itself; (3) the mapping $y=\alpha(x)$ of $X$ into $Y$ is a closed mapping; the sets $\alpha^{-1}(y)$ for $y \in Y$, are perfectly compact in themselves.

By Theorem 15.3 the conditions (2) and (1) are equivalent, respectively, to the upper semi-continuity of $X$ and of $Y$. Cf. E 15.1 .

The close resemblance of Theorems 15.1 and 15.4 fails to extend to the explicit assumption of the continuity of the mapping $y=\alpha(x)$, which is necessary for the former. The inherent potentialities for the continuity of $y=\alpha(x)$ for Theorem 15.4 are an example of the properties of this mapping which follow from the way it is defined: For $y \in Y, y<\alpha^{-1}(y)<y ; X \supset \alpha^{-1}(y)$; and if $x \in \alpha^{-1}(y)$, then $x<y$ (cf. D 9.3). These order relations, which involve $X, Y$, and the mapping, may be regarded as a key to our theory, and are a foundation for the applications we make in following sections. The transformation expresses the fact that the elements of $y$ are obtained by amalgamations of elements of $X$; conversely, the elements of $X$ are the results of decompositions of the $y$ 's. For topological applications we need conditions of continuity as well as those for order; simultaneous conditions for the required continuity and order are given in Theorems 15.3 to 15.6.

Proof. By Theorem 15.3 if condition (1) or (2) holds, then $Y$ or $X$, respectively, is upper semi-continuous relative to $S$. By (1) and (2) the mapping $y=\alpha(x)$ is continuous from $X$ to $Y$ (cf. argument on page 67, and Theorem IV, p. 53, Alexandroff and Hopf). The conclusion follows from Theorem 15.1.

Theorem 15.5. Adopt the notation of Theorem 15.4, and let $X$ be upper semicontinuous $\left({ }^{24}\right)$ relative to $S$; then (A) $Y$ is upper semi-continuous relative to $S$ if and only if the aggregate $Y^{\prime}=\left[\alpha^{-1}(y)\right]$, where $y \in Y$, is an upper semi-continuous collection of point sets in $\left.X{ }^{(25}\right)$. (B) Let $Y^{\prime \prime}=X\left(Y^{\prime}\right)$ be the space $\left({ }^{26}\right)$ of the decomposition of $X, Y^{\prime}$, and let $y^{\prime \prime}=\alpha^{\prime \prime}(x)$ mean that $x \in y^{\prime \prime} \in Y^{\prime \prime}$; then the mapping $y=\alpha(x)$ of $X$ on $Y$ is closed if and only if the mapping $y^{\prime \prime}=\alpha^{\prime \prime}(x)$ of $X$ on $Y^{\prime \prime}$ is closed. (C) If the conditions in either (A) or (B) are satisfied, then those in each of Theorem 15.4, (A) and (B), are satisfied, and the mappings $y=\alpha(x)$ and $y^{\prime \prime}=\alpha^{\prime \prime}(x)$ are equivalent mappings of $X\left({ }^{27}\right)$.

(24) Or, $X$ is perfectly compact in itself (cf. Theorem 15.3).

${ }^{(25)}$ Cf. definition (II). Here $X$ is regarded as a space (cf. Theorem 12.1).

${ }^{(26)}$ Cf. Definition (III); $Y^{\prime \prime}$ is der zur Zerlegung gehörende schwache Zerlegungsraum (cf. Alexandroff and Hopf, p. 66). The points of $Y^{\prime \prime}$ are the elements of $Y^{\prime}$.

(27) That is, the mapping, $y^{\prime \prime}=\alpha^{\prime \prime}\left(\alpha^{-1}(y)\right)$, of $Y$ on $Y^{\prime \prime}$ is a homeomorphism (cf. Alexandroff and Hopf, p. 61). 
Proof. By Theorems 14.7 and $12.1 X$ and $Y$ determine Hausdorff spaces. Let two of the conditions of Theorem 15.4 hold. The third follows; by Theorems VIII and II, pages 98 and 95, Alexandroff and Hopf, the mappings of (B) are equivalent and both are closed, and $Y^{\prime}$ is upper semi-continuous in $X$.

By Theorem 15.3 our hypothesis that $X$ is upper semi-continuous relative to $S$ is equivalent to condition (2) of Theorem 15.4. If $y=\alpha(x)$ is a closed mapping, condition (3) of that theorem holds. By the preceding paragraph the conditions in (A) and in (B) are necessary. Let $y^{\prime \prime}=\alpha^{\prime \prime}(x)$ be a closed mapping from $X$ to $Y^{\prime \prime}$. Let $y \in Y, U$ be an $S$-neighborhood of $y$, and $U(X)$ and $U(Y)$ denote all elements of $X$ and of $Y$, respectively of which $U$ is an $S$-neighborhood; $U(X)$ is open relative to $X$, and $U(X) \supset \alpha^{-1}(y)=y^{\prime \prime}$. If $V$ is the set of all elements of $Y^{\prime \prime}$ that are subsets of $U(X)$, then $V$ is an open set in $Y^{\prime \prime}$, and $y^{\prime \prime} \in V$ (Definition (III)). Let $H$ be the sum of the elements of $Y^{\prime \prime}-V$. Then $y^{\prime \prime}$ and $H$ are closed point sets in $X$ which do not intersect (cf. Theorem I, p. 53, Alexandroff and Hopf). Since $X$ is perfectly compact, so are $y^{\prime \prime}$ and $H$. By Theorems 14.10, 14.7, and $11.6 y^{\prime \prime}$ and $H$ can be separated by $S$-neighborhoods $D$ and $R$. Since $y^{\prime \prime}=\alpha^{-1}(y), y<y^{\prime \prime}<y$; by Theorem 11.6 $D$ is an $S$-neighborhood of $y$. Let $D$ be an $S$-neighborhood of a portion $\beta$ of an element of $Y, y_{1}$. Suppose that $y_{1} \in Y-U(Y)$; by Theorem 11.6 there exists $x_{1} \in \alpha^{-1}\left(y_{1}\right)$ such that $U$ is not an $S$-neighborhood of $x_{1}$. Then $\alpha^{-1}\left(y_{1}\right)$ $=\alpha^{\prime \prime}\left(x_{1}\right) \in Y^{\prime \prime}-V$, and $H \supset \alpha^{-1}\left(y_{1}\right)$. By Theorem $11.6 R$ is an $S$-neighborhood of $y_{1}$ and of $\beta$. Since $D$ is an $S$-neighborhood of $\beta$, and $R \cdot D$ is vacuous, we are involved in a contradiction. Thus, $Y$ is upper semi-continuous relative to $S$. By Theorems 15.3 and 15.4 the condition in (B) is sufficient. If the collection $Y^{\prime}$ is upper semi-continuous in $X$, it follows from Theorems VIII and II on pages 98 and 95, Alexandroff and Hopf, that the mapping $y^{\prime \prime}=\alpha^{\prime \prime}(x)$ is closed from $X$ to $Y^{\prime \prime}$. By (B) the mapping $y=\alpha(x)$ is closed. By the first paragraph the condition in (A) is sufficient, and (C) is true.

TheOREM 15.6. The conclusions of Theorems 15.3, 15.4, and 15.5 remain true if the aggregate consisting of $P$ and of the elements of $X$ and $Y$ include regular real or regular frontier points.

This follows from Theorems 12.1, 13.7, and 14.2.

16. Applications of the preceding sections. Applications of the preceding section require the decomposition of an amalgamation point $P$ into regular amalgamation points. In problems dealing with the embedding of $S$ in a perfectly compact Hausdorff space we have the case where $P$ is the maximal amalgamation point and the elements of the decomposition include the points of $S$ (cf. Theorem 16.1).

We supplement the results of $\S \S 13$ and 14 for the regular, the normal, and the semi-completely normal spaces by giving characterizations of the completely regular, the locally perfectly compact, and the perfectly compact 
Hausdorff spaces. Some of these give our interpretations of results by other authors, and demonstrate the generality of our methods (cf. Theorems 16.1, 16.2, and 16.4). In order to utilize this generality fully, we devote Chapter III to a study of lattices of regular points, and to lattices of systems of decompositions of an amalgamation point into collections of regular amalgamation points. These give systematic methods for classifying our results. An important part of Chapter III is the demonstration of the existence of atomic elements of systems of points which are encountered in applications of our mapping theory. For the special case of the semi-completely normal space the -equired atomic elements are the atomic ideal points (cf. Theorem 16.5). Similar conclusions hold for the decompositions of boundary points of normal spaces (cf. Theorem 16.1, (3)).

THEOREM 16.1. (1) In order that $S$ be completely regular $\left({ }^{28}\right)$, it is necessary and sufficient that there exist a collection of regular boundary points $M$ such that (a) each boundary point intersect $M$, and (b) the collection $S+M$ be upper semi-continuous relative to $S$. (2) This condition is satisfied if and only if $S+M$ is a perfectly compact Hausdorff space. (3) If $S$ is normal and $M$ is the set of its atomic boundary points, this condition is satisfied. (4) A perfectly compact, immediate Hausdorff extension of $S$ is a decomposition of the maximal ideal point.

The result in (3) is similar to that in Lemma 12, p. 119, Wallman, loc. cit. If $Y$ is any perfectly compact Hausdorff space in which $S$ is embedded and $X$ is the space $S+M$ of (3), then the conclusion of Theorem 15.4 concerning the mapping $y=\alpha(x)$ holds; cf. Stone, loc. cit., p. 476, Theorem 88. See also Theorem 20.2. Cech, also, loc. cit., has considered this space.

Proof. Let $Y$ be the maximal amalgamation point and $X=S+M$. Let $P$ be a real point, $Q$ be a boundary point, and $\beta$ be an atomic portion of $Q$. By (1a) $\beta$ intersects $\beta_{M} \in M$; since $\beta$ is atomic, $\beta<\beta_{M}$. Since $\beta_{M}$ is regular, $P$ and $\beta_{M}$ do not intersect, and they can be separated by $S$-neighborhoods $U$ and $V$ (cf. D 13.1 and Theorem 11.5). Since $\beta<\beta_{M}, V$ is an $S$-neighborhood of $\beta$ and $P$ and $\beta$ do not intersect (Theorems 11.6 and 11.5). By Theorem 14.2 $Q$ is an amalgamation point. By Theorem 14.6 $P$ and $Q$ do not intersect. By Theorem 13.1 $P$ is regular. By (1a) each composition point intersects either $S$ or $M$. By D $9.2 Y<M+S$; since $M+S<Y, X=S+M$ is a decomposition of $Y$ (cf. Theorem 15.2). By Theorems 14.5, 15.6, and 15.3 the condition in (1) is sufficient and that of (2) is necessary.

Conversely, let the perfectly compact Hausdorff space $T$ be an immediate extension of $S$; then all points of $T$ are regular. Let $M$ be the boundary points of $S$ which are equivalent to points of $T-S$. By Theorems 13.7 and 12.1,

(28) $S$ is completely regular if and only if it can be embedded in a perfectly compact Hausdorff space; cf. Tychonoff, loc. cit. 
$S+M$ is perfectly compact. By Theorems 14.10 and 14.2 there exists an amalgamation $Z$ of the elements of $S+M$. If $Z \neq Y$, there exists an atomic boundary point $\beta$ which intersects $Y$ but does not intersect $Z$ (cf. Theorem 9.2). By Theorems 12.1 and 14.7 the space $S+M+\beta$ is a Hausdorff space; clearly, $\beta$ is a limit point of $S$. Since $S+M$ is perfectly compact, we are involved in a contradiction; cf. Alexandroff and Hopf, p. 91, Theorem XI. Thus, $Y<Z$, $Z<Y$, and $Z=Y$. By Theorems 15.6 and 15.3 the condition in (1) is necessary, and that of $(2)$ is sufficient.

For Case (3) the collection $X=S+M$ obviously is upper semi-continuous. By Theorems 5.5 and 14.12 $M$ satisfies condition (1a).

THEOREM 16.2. (1) If $S$ is regular but is not perfectly compact, then in order that the set of all boundary points be amalgamable it is necessary and sufficient that $S$ be locally perfectly compact; (2) if this condition is satisfied and $Q$ is the amalgamation of all the boundary points, then $S+Q$ is perfectly compact.

This theorem resembles closely one due to Alexandroff; cf. Alexandroff and Hopf, p. 93, Theorem XIV.

Proof. If the condition holds, it follows from Alexandroff's theorem that there exists a point $Q$, such that $S+Q$ is a perfectly compact Hausdorff space in which $S$ is embedded; because of Theorems 13.7 and 12.1 we may suppose that $Q$ is a boundary point; by Theorem $14.2 Q$ is an amalgamation point. It follows from Theorem 16.1 that each boundary point is a portion of $Q$. Thus, the condition is sufficient.

Conversely, let $Q$ be the amalgamation of all the boundary points. By Theorems 13.1 and $14.6 Q$ and the real point $P$ can be separated by $S$-neighborhoods $U$ and $W$, respectively. If $\bar{W}$ were not perfectly compact, by Theorem 10.3 there would exist a boundary point $\beta$ which is an end of $\bar{W}$. Then $\beta$ is a portion of $Q$, and $U$ is an $S$-neighborhood of $\beta$. By Theorem $12.3 \beta$ is a limit point of $\bar{W}$, and $W \cdot U$ is nonvacuous. Thus, we are involved in a contradiction, and the condition is necessary.

THEOREM 16.3. In order that a Hausdorff space be perfectly compact, it is necessary and sufficient that it have no boundary points.

This follows from Theorem 10.3.

THEOREM 16.4. Let $X$ be a perfectly compact Hausdorff space which is an immediate extension of $S, P$ be the maximal $S$-portion, $M$ be a decomposition of $X$ into closed point sets, and $Y$ be the aggregate of all ideal points, $y(m)$, where $y(m)$ is the amalgamation of the elements of $m$, and $m \in M$. Theorems 15.4 and 15.5 are applicable.

Thus, $Y$ is upper semi-continuous relative to $S$ if and only if $M$ is upper semi-continuous in $X$; etc. The theorem points out that the results of Alexandroff and Hopf are special cases of our's. 
THEOREM 16.5. If $S$ is semi-completely normal, the results of Theorems 15.4 and 15.5 hold true for the case that $X$ is the decomposition of $P$ into atomic ideal points.

An interesting case is the one where $P$ is the maximal ideal point, and $Y$ is a perfectly compact Hausdorff space in which $S$ is embedded. This justifies our regarding $X$ as a universal inverse mapping space.

17. Extensions of upper semi-continuous collections of point sets in normal spaces. Stone has commented on the "remarkable properties" of a space which for the case that $S$ is normal is a homeomorph of $S$ plus its atomic boundary points (loc. cit., p. 476, lines 8 and 9). The results of this section give additional grounds for this comment. Our results in this section are distinguished by the fact that they are characteristic of normal spaces. Also, cf. Cech, loc. cit.

D 17.1. If $S$ is a subspace of $T, M$ and $N$ are collections of mutually exclusive closed point sets of $S$ and of $T$, respectively, and $M$ is the collection of all sets $S \cdot n$, where $n \in N$, we say that $N$ is an extension of $M$ (from $S$ into $T$ ).

E 17.1. Let $T$ be a circle plus its interior, and $S$ be the interior. Let $N$ be the set of all chords parallel to a given diameter; define $M$ as in D 17.1. $N$ contains an extension of $M, N$ is upper semi-continuous in $T$, but $M$ is not upper semi-continuous in $S$. Thus, $T$ cannot take the place of the space $\lambda(S)$ of Theorem 17.1.

Let $E$ and $F$ be two chords in $T$ such that $E$ and $F$ have in common exactly one point, $P$, which belongs to $T-S$. Let $K$ be the collection whose elements are $E \cdot S, F \cdot S$, and the points of $S-S \cdot(E+F)$. Then $K$ is upper semi-continuous in $S$, but cannot be extended to $T$. It follows that $T$ does not serve as a $T_{K}$ (cf. Theorem 17.2).

THEOREM 17.1. Let $S$ be normal, $\lambda(S)$ be the space of $S$ and its atomic boundary points, $M$ be a collection of mutually exclusive closed point sets of $S, N$ be the aggregate of the closures in $\lambda(S)$ of the elements of $S$, and $K$ be the aggregate of the amalgamations of the elements of $N$. (1) The following conditions are equivalent: (a) $M$ is upper semi-continuous in $S$; (b) $N$ is upper semi-continuous in $\lambda(S)$; (c) $K$ is upper semi-continuous relative to $S$. (2) If these conditions are satisfied, the spaces of these decompositions are homeomorphic $\left({ }^{29}\right)$.

Thus, the theory of upper semi-continuous collections of point sets in a normal space may be regarded as a part of the theory of our ideal points (this holds true even if the elements of $M$ are not perfectly compact).

Proof. For $m \in M$, let $n(m) \in N$ such that $m=S \cdot n(m)$; let $P(m)$ be the amalgamation of the elements of $n(m)$; by Theorems 16.1 and $14.10 P(m)$ exists. If $U$ is an open set in $S$, and $U \supset m \in M$, let $\lambda(U)$ denote all points of $\lambda(S)$ of which $U$ is an $S$-neighborhood. Suppose that $\beta \in n(m)-m$ and not

(29) Cf. Definition (III), $\$ 15$, and Theorem 12.1 . 
$\beta \in \lambda(U)$. Then by Theorems 11.3 and $12.3 \beta$ is an end of $S-U$ and of $m$. By Theorem 11.3 neither $U$ nor $S-m$ is an $S$-neighborhood of $\beta$. Since $S=U$ $+(S-m)$ and $S$ is an $S$-neighborhood of $\beta$, by Theorem 11.7 we are involved in a contradiction. Thus, $U$ is an $S$-neighborhood of $n(m)$ and of $P(m)$; cf. Theorem 11.6. Conversely, if $W$ is an open set in $\lambda(S)$, and $W \supset n(m)$, then $n(m)$ and $\lambda(S)-W$ are closed in $\lambda(S)$. Thus, $n(m)$ and $\lambda(S)-W$ are perfectly compact in themselves and are amalgamable (cf. Theorems 16.1 and 14.10); by Theorems 14.7 and 11.6 they can be separated by $S$-neighborhoods, $D$ and $R$. Then $W \supset \lambda(D) \supset n(m), D \supset m$, and $D$ is an $S$-neighborhood of $P(m)$. By Theorem 14.10 each $P(m)$ is regular. The conclusion follows from these relations, the definitions of upper semi-continuity, and Theorem IV, p. 53, Alexandroff and Hopf.

THEOREM 17.2. (1) In order that it be possible to extend each $M$, which is an upper semi-continuous decomposition of $S$ into closed point sets, to a subset of a similar decomposition of some immediate, perfectly compact, Hausdorff extension of $S$, say $T_{M}$, it is necessary and sufficient that $S$ be normal. (2) (a) If $S$ is normal, there exists a $T_{M}, Y$, which is independent of $M$; (b) such a $Y$ is homeomorphic to $X=\lambda(S)$ by means of the mapping $y=\alpha(x)$ of Theorems 15.6 and 15.4.

In particular, the conclusion of (2) holds for $Y=\lambda(S)$; cf. Theorem 17.1.

Proof. Let $E$ and $F$ be mutually exclusive closed sets in $S$. Let $M$ be the aggregate whose elements are $E, F$, and the points of $S-(E+F)$. Then $M$ is an upper semi-continuous decomposition of $S$. If a $T_{M}$ exists, there exists an upper semi-continuous extension of $M$ into $T_{M}$. Then the product of the closures of $E$ and $F$ in $T_{M}$ is vacuous. Since $T_{M}$ is perfectly compact, it is normal (cf. Alexandroff and Hopf, p. 89). There exist in $T_{M}$ mutually exclusive open sets, $U$ and $V$, which contain $\bar{E}_{T}$ and $\bar{F}_{T}$, respectively. Since $U \cdot S \supset E$ and $V \cdot S \supset F$, the condition in (1) is necessary.

Conversely, let $S$ be normal and $T$ be the space of the decomposition $M$ (cf. Definition (III), §15). If $t \in T$ and $s \in S$, let the relation $t=f(s)$ mean that $s \in t$ (recall that the points of $T$ are the elements of $M$ ). Then the mapping of $S$ on $T, t=f(s)$, is continuous and $T$ is normal (cf. Alexandroff and Hopf, pp. 67, 53, and 70). By Theorem 16.1 there exists an immediate, perfectly compact, Hausdorff extension of $T$, say $R$. There exists a continuous mapping from $\lambda(S)$ to $R$, say $r=F(s)$, such that if $P \in S$, then $f(P)=F(P)$ (cf. Theorem 16.1 and Stone, loc. cit., Theorem 88, p. 476$)$.

Let $N$ be the aggregate $\left[F^{-1}(r)\right]$, where $r$ ranges over $R$. By Theorem VIII, p. 98, Alexandroff and Hopf, $N$ is an upper semi-continuous decomposition of $Y=\lambda(S)$ into closed point sets. Thus, we have established (2a) and the sufficiency of the condition in (1).

Consider any $Y$ which is independent of $M$, and let $X$ be the space consisting of $S$ and those boundary points which are equivalent to points of $Y$; the mapping of the conclusion is a homeomorphism of $X$ and $Y$ (cf. Theorem 
13.7). Suppose that the element $\delta$ of $X-S$ is not atomic; then there exist two atomic boundary points which are portions of it, say $\alpha$ and $\beta$. By Theorem $14.12 \alpha$ and $\beta$ are regular. By Theorem 14.7 there exist $S$-neighborhoods of $\alpha$ and of $\beta$ whose closures in $S, E$ and $F$ are mutually exclusive. If $M$ is the collection whose elements are $E, F$, and the points of $S-(E+F)$, it is an upper semi-continuous decomposition of $S$. Clearly, $\alpha$ is a limit point of $E$ and $\beta$ is a limit point of $F$; by Theorem 11.6 each $S$-neighborhood of $\delta$ contains points of $E$ and of $F$, and $\delta$ is a limit point of $E$ and of $F$. Hence, there exist no mutually exclusive closed sets in $Y$ which contain $E$ and $F$, respectively. Since $Y$ contains an extension of $M$, we are involved in a contradiction. Thus, $\delta$ does not exist, and $X=\lambda(S)$.

\section{Chapter III. The LATTICE-MAPPING THEORY OF SYSTEMS OF REgUlaR POINTS}

Let $P$ be a regular amalgamation point, and let $X, Y$, and $y=\alpha(x)$ be such that the conditions of Theorem 15.4 are satisfied. Recall that for $y \in Y$, $X \supset \alpha^{-1}(y)$ and $y$ is an amalgamation of the elements of $\alpha^{-1}(y)$. Since the mapping $y=\alpha(x)$ deals both with order and with continuity, we may think of $Y$ as a mapping space or an amalgamation space for $X$, and of $X$ as an inverse mapping space or a decomposition space for $Y$. If $S$ is semi-completely normal, we have shown in Theorem 16.5 that if $X^{*}$ is the decomposition of $P$ into atomic ideal points, then $X^{*}$ serves as a common inverse mapping space for all the $Y$ 's which are upper semi-continuous, or perfectly compact, decompositions of $P$ into regular amalgamation points. That is, the elements of $X^{*}$ may be said to be atomic from the point of view of our mapping theory. By Theorem 14.12 for spaces other than the semi-completely normal the atomic mapping points will not, in general, be the atomic ideal points. For these more general spaces there is thus the question of the existence of such atomic elements, and that of the existence of decomposition spaces or of inverse mapping spaces.

In $\$ 19$ we give conditions for which these questions have answers in the affirmative. Our methods involve an order relation $X<Y$, where $X$ and $Y$ are decompositions of $P$ which satisfy the conditions of Theorem 15.4. The ordered set so obtained is a complete lattice and its zero is the required set of atomic mapping points. The sublattices of this lattice, and their zeros and units, give an extensive body of information which is not in Chapter II for the case even of the semi-completely normal space. Important sublattices are considered in $\$ 20$.

Section 18 is concerned with preliminary methods dealing with multiplicative systems and lattices of ideal points, and with the generation of such systems from collections of points. The zeros of the sublattices of $\$ 19$ are, in general, collections of atomic elements of systems of ideal points.

In Example 14.3 we established the existence of a space for which the only 
regular amalgamation point is the maximal ideal point $P$; for it $P=X=Y$, and the application of Theorem 15.4 is extremely limited.

18. The generation of multiplicative systems and lattices of amalgamation points. We develop methods for generating such systems from any collection of amalgamation points. Most of these do not require a hypothesis of regularity. In Theorem 18.5 we give conditions for regularity. In Theorem 18.4 we give conditions which make the generated set a lattice.

Note that if $N$ is a subsystem of $M$, an atomic element $\left(^{30}\right)$ of $N$ need not be an atomic element of $M$ (for instance, $N$ might consist of a single nonatomic element of $M$ ). The term atomic regular (amalgamation) point means an atomic element of the system of all regular amalgamation points; it should not be confused with the term regular atomic point (of $S$ ). Theorems 14.2 and 14.12 show that the terms are synonymous only for the semi-completely normal spaces.

D 18.1. The set of points $M$ is said to be almost-multiplicative provided that if $M \supset K$ and $K$ has a lower bound in the system of all ideal points, then the greatest lower bound of $K$ belongs to $M$. The following are examples of almost-multiplicative systems: all atomic points; all composition points; all amalgamation points; all regular composition points; if $S$ is completely regular, all regular amalgamation points (cf. Theorems 5.4, 14.2, 14.11, and 20.1).

D 18.2. If $M$ is almost-multiplicative but is not completely multiplicative $\left.{ }^{(31}\right)$, it becomes a complete multiplicative system $M+\bar{O}$ by the addition of a zero element $\bar{O}$; if $M$ is completely multiplicative, let $M+\bar{O}$ denote $M$, and let $\bar{O}$ denote its zero.

These systems find applications, among others, in establishing the existence of atomic elements. If $M$ is the system of all regular amalgamation points of the space of Example E 14.3, it has one element, the maximal ideal point of the space. This is the atomic element of the system. This example shows how far the atomic regular points may differ from the atomic points.

THEOREM 18.1. Let $M$ be an almost-multiplicative system of amalgamation points. (1) If $P \in M$, there exists an atomic element of $M$ which is a portion of $P$; (2) no two atomic elements of $M$ intersect; (3) $M$ is completely multiplicative if and only if it has exactly one atomic element (its zero).

Proof. By Hausdorff, (I), pp. 140-141, there exists a system of elements of $M$, say $K$, which contains $P$, is monotonic, and is not a proper subset of any monotonic subcollection of $M$. For $k \in K$ the set $\alpha(k)$ is perfectly compact in itself (cf. Theorems 9.1 and 14.4). By Theorem 9.2 the $\alpha(k)$ 's are the elements of a monotonic collection of point sets. There exists an atomic point $\delta$ which is common to all the $\alpha(k)$ 's (cf. Kuratowski and Sierpiński, and Moore,

$\left.{ }^{(30}\right)$ Cf. D 3.3.

(31) Cf. D 3.7, and MacNeille, loc. cit., pp. 429, 442, and 443. 
(II)). It follows that $\delta$ is a portion of each element of $K$. By D 18.1 there exists an element of $M, \beta$, which is the greatest lower bound of the elements of $K$. Let $\lambda$ be an element of $M$ such that $\lambda<\beta$, and let $k \in K$. Then $\lambda<\beta<k$. It follows that $K+\lambda$ is monotonic; because of the definition of $K, \lambda \in K$; then $\beta<\lambda$; since $\lambda<\beta, \lambda=\beta$, and $\beta$ is an atomic element of $M$. Since $P \in K, \beta<P$.

Let $\alpha$ and $\beta$ be intersecting atomic elements of $M$. By Theorem 8.1 and D 18.1 there exists an element of $M, \delta$, which is a lower bound of $\alpha$ and $\beta$. Since $\delta<\beta$ and $\beta$ is atomic, $\beta<\delta$, and $\beta=\delta$; similarly, $\delta=\alpha$. The conclusion of (2) and the necessity of the condition in (3) follow. If $M$ has exactly one atomic element, it follows by (1) that this element is a lower bound of each collection of elements of $M$; by D 18.1 the condition in (3) is sufficient.

D 18.3. If $K$ and $M(K)$ are collections of amalgamation points, $M(K)$ is an almost-multiplicative system, and $M(K)$ consists of those amalgamation points which are greatest lower bounds of subcollections of $K$, we say that $M(K)$ is generated by $K$; if $A(K)$ is the aggregate of all amalgamation points $P$ such that $P$ is the amalgamation of the elements of some subcollection of $M(K)$, we say that $A(K)$ is the additive system generated by $K$. If $x \in K, x$ is the lower bound of the pair $x$ and $x$; thus $M(K) \supset K$; similarly $A(K) \supset M(K)$. If $K$ is either the set of all composition points, or of all atomic ideal points, then $K=M(K)$; if $S$ is semi-completely normal, $A(K)$ is the set of all amalgamation points.

THEOREM 18.2. Each collection of amalgamation points $K$ generates an additive system $A(K)$ and an almost-multiplicative system $M(K) . A(K)$ is an almost-multiplicative system, and $A(K)$ and $M(K)$ have the same atomic elements.

Proof. Let $M(K)$ and $A(K)$ be defined as in D 18.3. Let $H$ be a subcollection of $M(K)$ that has a lower bound. Let $H=H_{1}+H_{2}$ such that $K \supset H_{1}$ and $M(K)-K \supset H_{2}$. Let $F$ be the product of all sets $\alpha(h)$, where $h \in H$ (cf. D 8.5). For $h \in H_{2}$ let $H(h)$ be a subcollection of $K$ such that $h$ is the greatest lower bound of the elements of $H(h)$; cf. D 18.3. Let $H_{3}$ be the sum of all the $H(h)$ 's, for $h \in H_{2}$. Let $G=H_{1}+H_{3}$, and let $E$ be the product of all the $\alpha(g)$ 's, where $g \in G$. Clearly, $E \supset F$. Suppose that $\beta \in E-F$; there exists $h \in H$ such that $\beta$ is not an element of $\alpha(h)$. Since $G \supset H_{1}, h \in H_{2}$; by Theorem 9.2 there exists $h_{3} \in H(h)$ such that $\alpha\left(h_{3}\right)$ does not contain $\beta$. Since $h_{3} \in G$, we are involved in a contradiction, and $\beta$ does not exist. Thus, $E=F$; by Theorem $9.2 P(F)$ is the greatest lower bound of the elements of $H$ and of $G$. Since $K \supset G$, it follows from the definition of $M(K)$ that $P(F) \in M(K)$ (by Theorem $14.11 P(F)$ is an amalgamation point). Thus, $M(K)$ is an almost-multiplicative system of amalgamation points, and is generated by $K$.

Next let $H$ be a subcollection of $A(K)$, and let $F$ be the product of all the sets $\alpha(h)$, for $h \in H$. If $h \in H$, there exists a subcollection of $M(K), M_{h}$, such that $h$ is the amalgamation of the elements of $M_{h}$ (cf. D 18.3). For $\beta \in F$ and $h \in H$, let $x(\beta, h)$ be an element of $M_{h}$ that intersects $\beta$. For a fixed $\beta$, let $x(\beta)$ 
be the greatest lower bound of the $x(\beta, h)$ 's, where $h$ ranges over $H$; by the first paragraph, $x(\beta) \in M(K)$. By Theorem 9.2Fつ ${ }(x(\beta)), x(\beta)<P(F)$, and $P(F)$ is the greatest lower bound of the elements of $H$. Let $X$ be the aggregate of all the $x(\beta)$ 's, for $\beta \in F$. By Theorems 8.1, 9.2, and D 9.2, $X<P(F)$, and $P(F)<X$. By Theorem 14.11 and D $14.2 P(F)$ is an amalgamation of the elements of $X$. Since $M(K) \supset X$, it follows that $P(F) \in A(K)$, and that $A(K)$ is an almost-multiplicative system.

Since $A(K) \supset M(K)$, and for $a \in A(K)$ there exists $m \in M(K)$ such that $m<a$, it follows that the two systems have the same atomic elements.

THEOREM 18.3. Let $K$ be a system of amalgamation points, and $M(K)$ be the almost-multiplicative system generated by it. Then $K$ and $M(K)$ generate the same additive system; and, if $K$ is almost-multiplicative, $M(K)=K$.

THEOREM 18.4. If $A(K)$ is the additive system generated by the collection of amalgamation points $K$, then $A(K)+\bar{O}$ is a complete lattice if and only if it is possible to amalgamate the elements of $K$. If the condition is satisfied, the unit of the lattice is the amalgamation of the elements of $K$.

Proof. Suppose that $A(K)+\bar{O}$ is a complete lattice; then it has a unit, $I$, which is an element of $A(K)$. Since $A(K) \supset K$, for $k \in K, k<I$; by $\mathrm{D} 9.2$, $K<I$. Since $I \in A(K)$, it follows from D 18.3 and D 14.2 that there exists a subset of $M(K)$, say $M$, such that $I<M$. Let $X$ be an ideal point that intersects $I$; by $\mathrm{D} 9.2 X$ intersects an element of $M$, say $m$. By the definition of $M(K)$ there exists $k \in K$ such that $m<k$. Then by D $9.2 X$ intersects $k$, and $I<K$; since $K<I$, it follows from $\mathrm{D} 14.2$ that $I$ is an amalgamation of the elements of $K$.

Conversely, let $I$ be an amalgamation of the elements of $K$. Since $M(K) \supset K, I \in A(K)$. Let $\beta \in A(K)$; then there exists a subcollection of $M(K)$, $M$, such that $\beta<M<\beta$. For $m \in M$ there exists $k \in K$ such that $m<k$; by D $9.2 M<K$; then $\beta<M<K<I$. Thus $I$ is the unit of $A(K)$. By Theorem 18.2 and $\mathrm{D} 18.2 A(K)+\bar{O}$ is a complete multiplicative system; since it has a unit, it is a complete lattice (cf. MacNeille, pp. 430-431).

THEOREM 18.5. If $K$ is a collection of regular amalgamation points, and each element of $K$ is a portion of some collection of regular composition points, then the additive and the multiplicative systems generated by $K$ consist of regular points.

Proof. If $k \in K$ and $\beta \in \alpha(k)$ it follows from the hypothesis and Theorem 9.2 that $\beta$ is a portion of some regular composition point $X$. By Theorems 9.2 , $14.2,14.11$, and 14.8 the greatest lower bound of $k$ and $X$ is a regular composition point $k(\beta)$, and $\beta<k(\beta)$. It can be shown with the help of Theorem 9.1 that $k<\alpha(k)<L<k$, where $L$ is the aggregate of all $k(\beta)$ 's. Thus, $k$ is an amalgamation of a collection of regular composition points. The conclusion follows from D 18.3 and Theorems 14.11 and 14.10 . 
19. Systems of upper semi-continuous, perfectly compact decompositions of regular amalgamation points. Let us consider the aggregate $D(R)$ of all decompositions of the perfectly compact Hausdorff space $R$ into upper semicontinuous collections of closed point sets. If $X$ and $Y$ are elements of $D(R)$ and each element of $X$ is a subset of some element of $Y$, let us say that $X<Y$. This relation orders $D(R)$. The following analogue to Theorem 19.1 holds: If $Y_{1}$ and $Y_{2}$ are elements of $D(R)$ and $X$ is the set of all products $y_{1} \cdot y_{2}$, where $y_{1} \in Y_{1}$ and $y_{2} \in Y_{2}$, then $X$ is the greatest lower bound of $Y_{1}$ and $Y_{2}$ in $D(R)$. Then $X$ is upper semi-continuous, and it corresponds to the $\omega_{M}(P)$ of Theorem 19.1, if $M$ is the pair $Y_{1}$ and $Y_{2}$. Such analogies may be extended to the case of arbitrary subcollections $M$ of $D(R)$. We shall now extend these ideas to the case of the amalgamation points.

D 19.1. If $P$ is a regular amalgamation point, let $\delta(P)$ be the set of all $\delta$ 's, where $\delta$ is an upper semi-continuous decomposition $\left({ }^{32}\right)$ of $P$ into regular amalgamation points. If $X$ and $Y$ are elements of $\delta(P)$, let the relation $X<Y$ mean that each element of $Y$ is decomposible into a set of points which is a subcollection of $X$; let $\delta(P)$ be ordered by this relation. Let $L(P)$ denote the sum of the elements of $\delta(P)$, and $\omega(P)$ be the set of atomic elements of $L(P)$.

Clearly this relation partially orders $\delta(P)$. The relation $X<Y$ is merely the requirement that $X$ and $Y$ satisfy the conditions of Theorem 15.4. Thus, the study of $\delta(P)$ and its sublattices systematizes our information about the mappings and inverse mappings we considered in $\$ \$ 15$ and 16 . We shall show that $\delta(P)$ is a complete lattice and that $\omega(P)$ is its zero. Thus, $\omega(P)$ may be mapped on any element of $\delta(P)$ by the methods of Theorem 15.4 ; the elements of $\omega(P)$ may be regarded as the atomic points from the point of view of these mappings. The zero of a sublattice of $\delta(P)$ has an analogous relation to the elements of the sublattice; and the elements of this zero may be regarded as the atomic points of the mapping theory which involves the elements of this sublattice.

EXAMPLE. E 19.1. Let $P$ be an amalgamation point in a semi-completely normal space $S$ or a boundary point of a normal space. Then $L(P)$ consists of all amalgamation points which are portions of $P$, and $\omega(P)$ is the decomposition of $P$ into atomic points (cf. Theorems 16.1, 16.5, and 14.13).

D 19.2. If $P$ is the maximal amalgamation point, let $\delta(S)=\delta(P)$ and $\omega(S)=\omega(P)$.

The preceding example suggests questions which the author has not solved for the case of the completely regular spaces. (I) If $A$ and $B$ are regular amalgamation points and $A<B$, does $\omega(B) \supset \omega(A)$ ? (II) Does $\omega(S)$ consist of the atomic regular points? An affirmative for (II) implies one for (I). A negative for (I) would imply the possibility of the existence of "incommensurable

$\left.{ }^{(32}\right)$ By Theorem 15.3 the condition of the upper semi-continuity of $\delta$ is equivalent to that of its perfect compactness (cf. D 9.3). 
points," $A$ and $B$; or that of a point $P$ and a point $\beta$ such that $\beta \in \omega(P)$ and $\omega(\beta)$ consists of proper portions of $\beta$. Theorem 14.12 shows that if $S$ is not semi-completely normal, some of its atomic points are too fine to be regular; the preceding discussion suggests that for more general spaces $S$ some of the atomic regular points may be too fine to belong to $\omega(S)$. In any case the existence of $\omega(P)$ is of importance, since it is the finest decomposition of $P$ for which the methods of $\$ 15$ are applicable.

THEOREM 19.1. Let $P$ be a regular amalgamation point which is a portion of a collection of regular composition points; let $M$ be a subcollection of $\delta(P)$; let $L_{M}(P)$ be the additive system which is generated by the sum of the elements of $M$, and $\omega_{M}(P)$ be the set of atomic elements of $L_{M}(P):(1) \omega_{M}(P) \in \delta(P)$; (2) if $\beta \in \omega_{M}(P)$ and $N \in M, \beta$ intersects exactly one element of $N$, say $N_{\beta}$; and $\beta$ is the greatest lower bound of all the $N_{\beta}$ 's; (3) $L_{M}(P)$ is the sum of all the $Y$ 's such that $Y \in \delta(P)$ and $\omega_{M}(P)<Y$ in $\delta(P)$; the elements of $M$ are such $Y$ 's; (4) $L_{M}(P)+\bar{O}$ is a complete lattice of regular amalgamation points, and its unit is $P$.

The lattice of (4) is obtained by the methods of the preceding section. The set of its atomic elements, $\omega_{M}(P)$, is the zero of a certain sublattice of $\delta(P)$; this lattice is suggested in condition (3) (cf. Theorem 19.3). This condition is used to show that the systems in the following two theorems are complete multiplicative systems.

Proof. Let $\beta$ be an atomic portion of $P$; it intersects exactly one element, $N_{\beta}$, of each element $N$ of $M$. By Theorem $9.2, \beta<P_{\beta}$, the greatest lower bound of all the $N_{\beta}$ 's. By D $18.3 P_{\beta} \in L_{M}(P)$; by Theorem $18.5 P_{\beta}$ is regular. By Theorems 18.1 and 18.2 there exists an atomic element of $L_{M}(P)$, say $A$, which is a portion of $P_{\beta}$; and there exists a subcollection of the sum of the elements of $M$, say $A^{*}$, such that $A$ is the greatest lower bound of $A^{*}$. Since $A<P_{\beta}, A$ is a lower bound of the elements of $\left[N_{\beta}\right]$. If $N \in M$, no two elements of $N$ intersect, since $N$ is a decomposition of $P$ into amalgamation points. It follows that $\left[N_{\beta}\right] \supset A^{*}$; by Theorem $9.2 P_{\beta}<A$; since $A<P_{\beta}, A=P_{\beta}$; thus we have established (2). Since $\beta<P_{\beta} \in \omega_{M}(P)$, it follows with the help of Theorems 9.1 and 9.2 that $P<\alpha(P)<\omega_{M}(P)<P$; by Theorem 18.1 no two elements of $\omega_{M}(P)$ intersect; thus $\omega_{M}(P)$ is a decomposition of $P$. It remains to show that $\omega_{M}(P)$ is upper semi-continuous.

First we shall establish this upper semi-continuity for the case that the number $\lambda$ of elements of $M$ is a positive integer. Let $n=\lambda$ be a positive integer such that the conclusion holds for each collection of $n$ elements of $\delta(P)$; clearly, $n=1$ is such an integer. Let $M$ and $H$ be two subcollections of $\delta(P)$ which have, respectively, $n+1$ and $n$ elements, and are such that $M \supset H$. Let $J=\omega_{H}(P), K$ be the element of $M-H$, and $L$ be the set of atomic elements of the additive system generated by $J+K$. It follows from part (2) 
and Theorem 9.2 that $L=\omega_{M}(P)$. Let $\beta \in L$ and let $D$ be an $S$-neighborhood of $\beta$. There exist elements of $J$ and $K$, say $j$ and $k$, such that $\beta$ is the greatest lower bound of $j$ and $k$. By an argument used in the proof of Theorem 14.11 there exist in $S$ mutually exclusive open sets $D_{1}$ and $D_{2}$ such that $D+D_{1}$ and $D+D_{2}$ are $S$-neighborhoods, respectively, of $j$ and of $k$. Since $J$ is upper semicontinuous, there exists an $S$-neighborhood of $J, R_{1}$, such that if $R_{1}$ is an $S$-neighborhood of a portion of an element of $j$, then $D+D_{1}$ is an $S$-neighborhood of that element. Similarly define $R_{2}$ for $k$ and $D+D_{2}$; let $R=R_{1} \cdot R_{2}$. Let $R$ be an $S$-neighborhood of a portion of an element of $L, \beta_{1}$, which is the greatest lower bound of $j_{1}$ and $k_{1}$. Then $D+D_{1}$ and $D+D_{2}$ are $S$-neighborhoods of $j_{1}$ and of $k_{1}$. Since $D_{1} \cdot D_{2}$ is vacuous, it follows from Theorems 9.2 and 11.6 that $D$ is an $S$-neighborhood of $\alpha\left(\beta_{1}\right)=\alpha\left(j_{1}\right) \cdot \alpha\left(k_{1}\right)$ and of $\beta_{1}$. Thus $L$ is upper semi-continuous, and our conclusion holds for any finite case.

Let $M$ be infinite. Again let $\beta \in \omega_{M}(P)$ and $D$ be an $S$-neighborhood of $\beta$. By (2) $\beta$ is the greatest lower bound of the elements of $\left[N_{\beta}\right]$; by Theorem 9.2 $\alpha(\beta)$ is the product of the sets $\alpha\left(N_{\beta}\right)$. By methods used in the proof of Theorem 14.11 there exist a finite collection of the $N_{\beta}$ 's, $H=\left(N_{1 \beta}, N_{2 \beta}, \cdots, N_{k \beta}\right)$ such that $D$ is an $S$-neighborhood of the product $X=\alpha\left(N_{1 \beta}\right) \cdot \alpha\left(N_{2 \beta}\right) \cdots \alpha\left(N_{k \beta}\right)$. If $\beta_{k}$ is the greatest lower bound of the elements of $H$, by Theorems 9.2 and 11.6 $D$ is an $S$-neighborhood of $\beta_{k}$. Let $N_{i \beta} \in N_{i} \in M, G=\left(N_{1}, N_{2}, \cdots, N_{k}\right)$, and $L=\omega_{G}(P)$. By the preceding paragraph $L \in \delta(K)$ and $\beta_{k} \in L$. Since $L$ is upper semi-continuous, there exists an $S$-neighborhood of $\beta_{k}, R$, such that if $R$ is an $S$-neighborhood of a portion of an element of $L$ then $D$ is an $S$-neighborhood of that element. By Theorem $9.2 \beta<\beta_{k}$; by Theorem $11.6 R$ is an $S$-neighborhood of $\beta$. By condition (2) each element of $\omega_{M}(P)$ is a portion of an element of $L$; it follows that $\omega_{M}(P)$ is upper semi-continuous. We have established (1).

Let $Z$ be the almost-multiplicative system which is generated by the sum of the elements of $M$. By Theorem 18.2 $\omega_{M}(P)$ is the set of atomic elements of $Z$. Let $Y \in \delta(P)$ and $\omega_{M}(P)<Y$ in $\delta(P)$; by D 19.1 the latter means that each element of $Y, y$, is an amalgamation of a subcollection of $\omega_{M}(P)$. Since $Z \supset \omega_{M}(P), L_{M}(P) \supset Y$ (cf. D 18.3). Thus, if $\Sigma$ is the sum of all $Y$ 's that satisfy the condition of (3), then $L_{M}(P) \supset \Sigma$. Conversely, let $A \in L_{M}(P)$ and let $H$ be the set of all elements of $\omega_{M}(P)$ that are portions of $A$; then $A$ is an amalgamation of the elements of $H$. Let $L$ be the set which consists of $H$ and the elements of $\omega_{M}(P)-H$; let $Y$ consist of $A$ and the elements of $\omega_{M}(P)-H$. Since $L_{M}(P)$ is an almost-multiplicative system and its atomic elements are the elements of $\omega_{M}(P)$, no element of $\omega_{M}(P)-H$ intersects $A$ (cf. Theorems 18.1 and 18.2). By Theorem 14.7 $A$ and $H$ can be separated from each element of $\omega_{M}(P)-H$ by $S$-neighborhoods, and none of these elements is a limit point of $H$. Thus, $H$ is closed relative to $\omega_{M}(P)$. By Theorem $15.3 \omega_{M}(P)$ is perfectly compact in itself. Since $L$ is an upper semi-continuous decomposition of $\omega_{M}(P)$ into closed point sets, it follows from Theorem 15.5 that $Y$ is upper semi-con- 
tinuous relative to $S$. Then $Y \in \delta(P)$ and $\omega_{M}(P)<Y$ in $\delta(P)$. Thus, $\Sigma \supset L_{M}(P)$; since $L_{M}(P) \supset \Sigma, L_{M}(P)=\Sigma$.

The conclusion of (4) follows from Theorems 18.4 and 18.5.

TheOREM 19.2. Let $P$ be the point of Theorem 19.1. If $M=\delta(P)$, then $L(P)=L_{M}(P)$ and $\omega(P)=\omega_{M}(P)$. Also, $\delta(P)$ is a complete lattice with unit $P$ and zero $\omega(P)$.

Proof. If $M=\delta(P)$, by Theorem 19.1 and D $19.1 L_{M}(P) \supset L(P)$, and conversely. Thus, $L_{M}(P)=L(P)$ and $\omega_{M}(P)=\omega(P)$. By Theorem 19.1, (3), if $Y \in \delta(P)$, then $\omega(P)<Y$ in $\delta(P)$. Thus, $\omega(P)$ is the zero of $\delta(P)$; clearly $P$ is the unit of $\delta(P)$.

By Theorem 19.1 if $\delta(P) \supset M$ and $Y \in M$, then $\omega_{M}(P)<Y$ in $\delta(P)$. Let $X$ be a lower bound of $M$ in $\delta(P)$. If $x \in X$ and $N \in M$, there exists one element of $N, N_{x}$, such that $x$ is a portion of $N_{x}$ (cf. D 19.1). By (2) of Theorem 19.1 there exists an element of $\omega_{M}(P)$, say $\beta_{x}$, which is the greatest lower bound of the $N_{x}$ 's. Thus, $x$ is a portion of $\beta_{x}$. From this relation and the fact that each of $X$ and $\omega_{M}(P)$ is a decomposition of $P$, each element of $\omega_{M}(P)$ can be decomposed into a subcollection of $X$. Thus, $X<\omega_{M}(P)$ and $\omega_{M}(P)$ is the greatest lower bound of $M$ in $\delta(P)$. Since $\delta(P)$ has a unit, it is a complete lattice (cf. MacNeille, pp. 430, 431, and Birkhoff, p. 17).

Theorem 19.3. If $\delta(P) \supset M$ and $P$ satisfies the condition of Theorem 19.1, let $\omega_{1}(M, P)=\omega(P), \omega_{2}(M, P)=\omega_{M}(P), \omega_{3}(M, P)$ be the least upper bound of $M$ in $\delta(P)$, and $\omega_{4}(M, P)=P$; if $1 \leqq i \leqq j \leqq 4$, let $\delta_{i j}(M, P)$ be the set of all elements of $\delta(P), X$, such that $\omega_{i}(M, P)<X<\omega_{j}(M, P)$ in $\delta(P)$. Then $\delta_{i j}(M, P)$ is a complete sublattice of $\delta(P)$ and its zero and unit are, respectively, $\omega_{i}(M, P)$ and $\omega_{j}(M, P) ; \omega_{M}(P)$ is the greatest lower bound of $M$ in $\delta(P)$.

Thus, any $X<\omega_{2}(M, P)$ may serve as a common inverse mapping space for the elements of $M$. However, $X=\omega_{2}(M, P)$ may be regarded as the "most economical" of these inverse spaces. For, if $X \in \delta_{12}(M, P)$ and $Y_{1}$ and $Y_{2}$ are elements of $M$, we can first decompose the elements of $Y_{1}$ into points of $X$ and then reamalgamate these into points of $Y_{2}$ by the methods of Theorem 15.4. Since the elements of $X=\omega_{2}(M, P)$ are "coarser" than those of any other element of $\delta_{12}(M, P)$, the initial decomposition need not be extended so far if this $X$ is used for an inverse space. Similarly, $X=\omega(P)$ is the "least economical" of these inverse spaces, but it serves for all sets in $\delta(P)$. Analogous interpretations hold for the common mapping spaces of the elements of $M$, the elements of $\delta_{34}(M, P)$. Theorem 19.3 may be modified by replacing $\delta(P)$ by any of its complete sublattices.

20. Applications to completely regular spaces. If we order all the perfectly compact immediate Hausdorff extensions of $S$ by the ordering of D 19.1, we obtain a quasi-partially ordered system (cf. Theorems 13.7 and 16.1). By 
identifying $\left({ }^{33}\right)$ equivalent elements of this system, we obtain a complete multiplicative system which is isomorphic to the subsystem of $\delta(S), H(S)$, which we consider in D 20.1 and Theorems 20.2 to 20.4. The zero of $H(S), \lambda(S)$, is equivalent to the space considered by Stone in his Theorem 88, p. 476 . In $\$ 17$ we considered this space for the case that $S$ is normal (cf. also, Čech, loc. cit.)

The important theorems $14.11,18.5$, and 19.1 to 19.3 involve hypotheses in which certain points satisfy the condition of being the amalgamation of a collection of regular composition points $\left({ }^{34}\right)$. This condition and the results of the preceding section enter into the characterizations of completely regular spaces which are given in Theorem 20.1. The fact that the atomic mapping points in such a space are necessarily regular composition points gives an analogy to the results of Theorem 14.13.

D 20.1. Let $H(S)$ be the subsystem of $\delta(S)$ which consists of all $Y$ 's such that each point of $Y$ is either a boundary point or is equivalent $\left({ }^{35}\right)$ to a point of $S$. Let $\lambda(S)$ be the zero of $H(S)$ (cf. D 19.2).

TheOREM 20.1. Any of the following is a necessary and sufficient condition that $S$ be completely regular: (1) At least one element of $\delta(S)$ consists of composition points; (2) the elements of $\omega(S)$ are composition points; (3) if $Q$ is a regular amalgamation point, the elements of $\omega(Q)$ are regular composition points.

Any regular amalgamation point in a completely regular space is the amalgamation of some collection of regular composition points ${ }^{(36)}$.

Proof. By Theorems 13.7 and 16.1 the condition in (1) is necessary. Let $Y$ be an element of $\delta(S)$ which consists of regular composition points, $Q$ be a regular amalgamation point, and $K$ be the aggregate of all points $Q_{y}$, where $Q_{y}$ is the greatest lower bound of $Q$ and the element $y$ of $Y$. By Theorem 14.11 if $y$ intersects $Q$, then $Q_{y}$ is a regular amalgamation point. Since $Y$ is a decomposition of the maximal amalgamation point $P$, and $Q<P$, it may be shown that $K$ is a decomposition of $Q$. We may regard $Q$ as an upper semicontinuous collection of one element; since $Y$ is upper semi-continuous, we may show by an argument like that used for the finite case in the proof of Theorem 19.1 that $K$ is upper semi-continuous relative to $S$. By D 19.1 $K \in \delta(Q)$. By Theorem 19.1 if $\beta \in \omega(Q)$, there exist elements of $K$ and of $Y$, respectively, $k_{\beta}$ and $y_{\beta}$, such that $\beta<k_{\beta}<y_{\beta}$. Since $\beta$ is a regular amalgamation point and $y_{\beta}$ is a regular composition point, it follows from Theorem 14.8 that $\beta$ is a composition point. Thus, the conditions in (2) and (3) are necessary. Since $\omega(Q) \in \delta(Q)$, by $\mathrm{D} 19.1, \mathrm{D} 14.2$, and $\mathrm{D} 9.3 Q$ is an amalgamation of $\omega(Q)$.

( $\left.{ }^{33}\right)$ Cf. Birkhoff, p. 7, Theorem 1.2.

$\left.{ }^{(34}\right)$ There exist examples which show that such an hypothesis is essential for the truth of these theorems.

${ }^{(35)}$ Cf. D 3.2 and Theorem 13.7.

$\left({ }^{36}\right)$ Cf. Theorem 14.13. 
Clearly, each of (2) and (3) implies (1) (cf. Theorem 14.5). Let $X$ be an element of $\delta(S)$ which consists of regular composition points, $M$ be the set of those elements of $X$ which are boundary points, and $Y=S+M$. By Theorem 5.2 no boundary point intersects a decomposition point. By D 19.1, D 19.2, and D 9.2 each boundary point intersects $M$. By the argument used at the beginning of the proof of Theorem 16.1 it follows that the points of $S$ are regular. Since no two elements of $Y$ intersect, it follows from Theorems 14.6, 14.7, and 12.1 that $Y$ is a Hausdorff space. Let the mapping $y=\alpha(x)$ be defined as in Theorems 15.4 and 15.6. Let $\beta \in Y$, and $D$ be an $S$-neighborhood of $\beta$. Since $\beta$ is regular, there exists an $S$-neighborhood of $\beta, R$, such that $D \supset \bar{R}_{S}$. Let $x$ be an element of $X$ such that $R$ is an $S$-neighborhood of $x$. By Theorem 12.4 $\bar{R}_{S} \supset a(x)$. By D 12.2 and the definition of the mapping, $y=\alpha(x)$, either $x=\alpha(x)$ or $a(x)=\alpha(x)$; in either case $D$ is an $S$-neighborhood of $\alpha(x)$. Thus, our mapping is continuous from $X$ to $Y$ (cf. Alexandroff and Hopf, p. 53, Theorem IV). By Alexandroff and Hopf, Theorem VIII, p. 98, $Y$ is perfectly compact. By Theorems 15.3 and 16.1 the condition in (1) is sufficient.

THEOREM 20.2. Let $S$ be completely regular: (1) $H(S)$ is a complete multiplicative subsystem of $\delta(S) ;(2) \lambda(S)$ consists of the composition points which are equivalent to the points of $S$, and of the boundary points which are elements of $\omega(S)$; (3) if $X=\lambda(S)$ and $Y \in H(S)$, then $X$ may be mapped on $Y$ by the mapping $y=\alpha(x)$ of Theorem 15.4 .

Concerning (3) cf. Stone, loc. cit., Theorem 88.

Proof. Let $H^{\prime}$ denote the set of composition points which are equivalent to points of $H$ (cf. Theorem 13.7 and D 3.2). Let $M$ be a subcollection of $H(S)$. By Theorem $19.3 \omega_{M}(S)$ is the greatest lower bound of $M$ in $\delta(S)$. Since $S^{\prime}$ is a subset of each element of $M$, and the remaining points of each element of $M$ are boundary points, by Condition (2) of Theorem 19.1 $\omega_{M}(S) \supset S^{\prime}$, and $\omega_{M}(S)-S^{\prime}$ consists of boundary points (cf. Theorem 14.8). Thus, $\omega_{M}(S) \in H(S)$; (1) follows by D 3.7. If $M=H(S)$, by Theorem 19.3 and D $20.1 \lambda(S)=\omega_{M}(S)$. Let $K$ be the set of all boundary points which belong to $\omega(S)$, and let $Y=S^{\prime}+K$. By an argument similar to that used in the proof of the sufficiency of the condition of Theorem 20.1 it follows that $Y \in \delta(S)$; hence, $Y \in H(S)$. Thus, $\lambda(S)<Y$ in $H(S)$. Since $\omega(S)<\lambda(S)$ in $\delta(S)$, it follows that $\lambda(S)-S^{\prime}=K$, and that $\lambda(S)=Y$.

D 20.2. Let the symbol $H^{\prime}$ denote the set of all composition points which are equivalent to elements of $H$ (cf. D 3.2 and Theorem 13.7). Let $B$ be the set of all boundary points and $C$ be the set of all real points which are limit points of $B$. Let $Q(S)$ be the amalgamation of the elements of $B+C$. Let $I(S)=\left(S^{\prime}-C^{\prime}\right)+Q(S)$.

Recall that $H(S)$ contains the topological images of all immediate, perfectly compact Hausdorff extensions of $S$. Theorem 20.2 shows that $\lambda(S)$ is a universal inverse mapping space for such extensions, and is one of them. The 
two following theorems show that $I(S)$ is the least common mapping space of the elements of $H(S)$, and that it does not necessarily belong to $H(S)$. The following theorem gives the structure of $I(S)$. In particular, if $S$ is not locally perfectly compact at any of its points, then $Q(S)$ is $I(S)$ and is the maximal amalgamation point. Then $Q(S)$ is the unit of $\delta(S)$.

THEOREM 20.3. Let $S$ be completely regular. (1) $I(S)$ is the least upper bound of $H(S)$ in $\delta(S)$; (2) $I(S)-Q(S)$ consists of those composition points which are equivalent to the points of $S$ at which $S$ is locally perfectly compact.

Proof. Let $M$ be the set of all boundary points, $N$ be the set of real points which are limit points of $M$, and $K=S-N$. If $X \in K$, there exists an $S$-neighborhood of $X, R$, which is not an $S$-neighborhood of any element of $M+N$ (cf. Theorems 12.2 and 13.1). Let $D$ be an $S$-neighborhood of $X$ such that $R \supset \bar{D}$. If $\bar{D}$ were not perfectly compact, there would exist a boundary point $\beta$ such that $\beta$ is an end of $\bar{D}$ (Theorem 10.3). Then, since $R \supset \bar{D}$, it may be shown with the help of Theorem 10.1 that $R$ is an $S$-neighborhood of $\beta$; since $\beta \in M$, we are involved in a contradiction. It follows that if the composition point $\delta$ is a limit point of $M+N$, then $X \neq a(\delta)$ (cf. D 12.2). Thus, either $a(\delta) \in N$, or $a(\delta)$ is vacuous, and $\delta$ is a boundary point; in either case $\delta$ intersects an element of $M+N$. By Theorems 14.1 and $13.7 Q(S)$ is an amalgamation point. Clearly, no two elements of $I(S)$ intersect, each ideal point intersects an element of $I(S)$, and $I(S)$ is upper semi-continuous relative to $S$ (cf. D 15.1 and $\mathrm{D}$ 19.2). Thus, $I(S) \in \delta(S)$.

Let $Y \in H(S)$ and $Z$ be an upper bound of $H(S)$ in $\delta(S)$. Clearly, $Y<I(S)$ in $\delta(S)$. If $A$ and $B$ are boundary points which belong to elements of $H(S)$, they can be decomposed into subcollections of $\lambda(S), H_{A}$ and $H_{B}$; and they can be amalgamated into a point $C$ such that $C<H_{A}+H_{B}<C$. Let $L=C+\lambda(S)$ $-\left(H_{A}+H_{B}\right)$. Then $C$ intersects no element of $L-C$; since $\lambda(S)$ contains $L-C$ and is upper semi-continuous, it follows that $L$ is upper semi-continuous; also, $L$ is a decomposition of the maximal amalgamation point. Thus, $L$ is an element of $\delta(S)$ and of $H(S)$, and $L<Z$. Let $\delta \in Z$ such that $C$ is a portion of $\delta$; since $A$ and $B$ are arbitrary, it follows that $\delta$ is independent of $A, B$, and $C$; thus, by D $9.2 M$ is a portion of $\delta$. Since the elements of $N$ are limit points of $M$, they intersect $\delta$ (cf. Theorem 14.6). Since $S^{\prime} \supset N^{\prime}$, each element of $S^{\prime}$ belongs to each element of $H(S)$, and no two elements of $Z$ intersect, each element of $N$ is a portion of some element of $Z$; that is, of $\delta$. By D $9.2 Q(S)$ is a portion of $\delta$. Since the elements of $I(S)-Q(S)$ belong to $S^{\prime}$, it follows that $I(S)<Z$ in $\delta(S)$. We have established the conclusion.

THEOREM 20.4. (1) In order that $H(S)$ be a complete sublattice of $\delta(S)$, it is necessary and sufficient that $S$ be locally perfectly compact. (2) If this condition is satisfied, $\lambda(S)$ and $I(S)$ are the zero and the unit of $H(S)$; and $Q(S)$ is the amalgamation of all the boundary points. 
Here $I(S)$ is the topological image of the space we considered in Theorem 16.2. Cf. Alexandroff and Hopf, p. 93.

\section{BIBLIOGRAPHY}

Alexandroff, Paul, Über stetige Abbildungen kompakter Räume, Mathematische Annalen, vol. 96 (1926), pp. 555-571.

Alexandroff, P., and Hopf, H., Topologie, Berlin, Springer, 1935.

Alexandroff, P., and Urysohn, P., (I) Zur Theorie der topologischen Räume, Mathematische Annalen, vol. 92 (1924), pp. 258-266; (II) Mémoire sur les espaces topologicues compacts, Verhandelingen der Koninklijke Akademie van Wetenschappen, Amsterdam, vol. 14 (1929), pp. 1-96.

Birkhoff, Garrett, Lattice Theory, American Mathematical Society Colloquium Publications, vol. 25, New York, 1940.

Carathéodory, C., Über die Begrenzung einfach zusammenhängender Gebiete, Mathematische Annalen, vol. 73 (1913), pp. 323-370.

Cartan, H., (I) Théorie des filtres, Comptes Rendus de l'Académie des Sciences, vol. 205 (1937), pp. 595-598; (II) Filtres et ultrafiltres, ibid., pp. 777-779.

Čech, E., On bicompact spaces, Annals of Mathematics, (2), vol. 38 (1937), pp. 823-844.

Fréchet, M., (I) Les Espaces Abstraits et Leur Théorie Considerée comme Introduction a l'Analyse Générale, Paris, 1928; (II) Sur les ensembles abstraits, Annales de l'École Normale Supérieure, vol. 38 (1921), pp. 341-387.

Hausdorff, F., (I) Grundzüge der Mengenlehre, 1914; (II) Mengenlehre, 1927.

Jones, F. Burton, Concerning normal and completely normal spaces, Bulletin of the American Mathematical Society, vol. 43 (1937), pp. 673-675.

Kuratowski, C., Topologie, 1933.

Kuratowski, C., and Sierpiński, W., Le théorème de Borel-Lebesgue dans la théorie des ensembles abstraits, Fundamenta Mathematicae, vol. 2 (1921), pp. 172-179.

MacNeille, H. M., Partially ordered sets, these Transactions, vol. 42 (1937), pp. 416-460.

Menger, K., (I) Dimensionstheorie, 1928, and (II) Kurventheorie, 1932.

Moore, R. L., (I) Foundations of Point Set Theory, American Mathematical Society Colloquium Publications, vol. 13, 1932; (II) On the most general class L Fréchet in which the Heine-Borel-Lebesgue theorem holds true, Proceedings of the National Academy of Sciences, vol. 5 (1919), pp. 206-210; (III) Concerning upper semi-continuous collections of continua, these Transactions, vol. 27 (1925), pp. 416-428.

Sierpiński, W., Introduction to General Topology, 1934, translated by C. C. Krieger.

Stone, M. H., Applications of the theory of Boolean rings to general topology, these Transactions, vol. 41 (1937), pp. 375-481.

Tietze, H., Beiträge zur allgemeinen Topologie, Mathematische Annalen, vol. 88 (1923), pp. 290-312.

Tychonoff, A., Über die topologische Erweiterung von Räumen, Mathematische Annalen, vol. 102 (1929-1930), pp. 545-561.

Urysohn, P., Über die Mächtigkeit der zusammenhängender Mengen, Mathematische An' nalen, vol. 94 (1925), pp. 263-295.

Wallman, H., Lattices and topological spaces, Annals of Mathematics, (2), vol. 39 (1938), pp. 112-126.

University of TeXas, Austin, Texas. 